\title{
Cell type-specific histone acetylation profiling of Alzheimer's Disease subjects and integration with genetics
}

Easwaran Ramamurthy ${ }^{1 *}$, Gwyneth Welch ${ }^{2 *}$, Jemmie Cheng $^{2}$, Yixin Yuan ${ }^{1}$, Laura Gunsalus ${ }^{1}$, David A. Bennett $^{3}$, Li-Huei Tsai ${ }^{2 * *}$, Andreas Pfenning $1 * *$

1. Computational Biology Department, Carnegie Mellon University, Pittsburgh PA 15213

2. Picower Institute for Learning and Memory, Massachusetts Institute of Technology, Cambridge MA 02139

3. Rush University Medical Center, Chicago IL 60612

We profile genome-wide histone 3 lysine 27 acetylation (H3K27ac) of 3 major brain cell types from hippocampus and dorsolateral prefrontal cortex (dlPFC) of subjects with and without Alzheimer's Disease (AD). We confirm that single nucleotide polymorphisms (SNPs) associated with late onset AD (LOAD) prefer to reside in the microglial histone acetylome, which varies most strongly with age. We observe acetylation differences associated with $\mathrm{AD}$ pathology at 3,598 peaks, predominantly in an oligodendrocyte-enriched population. Strikingly, these differences occur at the promoters of known early onset AD (EOAD) risk genes (APP, PSEN1, PSEN2, BACE1), late onset AD (LOAD) risk genes (BIN1, PICALM, CLU, ADAM10, ADAMTS4, SORL1 and FERMT2), and putative enhancers annotated to other genes associated with AD pathology (MAPT). More broadly, acetylation differences in the oligodendrocyte-enriched population occur near genes in pathways for central nervous system myelination and oxidative phosphorylation. In most cases, these promoter acetylation differences are associated with differences in transcription in oligodendrocytes. Overall, we reveal deregulation of known and novel pathways in $\mathrm{AD}$ and highlight genomic regions as therapeutic targets in oligodendrocytes of hippocampus and dlPFC.

\section{INTRODUCTION:}

Alzheimer's Disease (AD) is the most common age-related neurodegenerative disorder ${ }^{1}$. The hallmarks of $\mathrm{AD}$ pathology are numerous and include neuronal loss, synaptic dysfunction, gliosis, and the accumulation of intercellular plaques of amyloid- $\beta(\mathrm{A} \beta)$ protein and intracellular neurofibrillary tangles (NFT) of phosphorylated tau protein $(M A P T)^{2}$.

$\mathrm{A} \beta$ plaques are formed by differential proteolytic cleavage of the amyloid $\beta$ precursor protein $(A P P)^{3-6}$ by the $\alpha$-secretase, $\beta$-secretase and $\gamma$-secretase enzymes ${ }^{7}$. Studies of individuals affected by early onset $(<60$ yrs.) familial AD (EOAD) have identified causal autosomal dominant mutations primarily in $\mathrm{A} \beta$ processing proteins presenilin-1 (PSEN1) and presenilin-2 (PSEN2), which are part of the $\gamma$-secretase complex $^{8-10}$, but also causal mutations or duplications in APP itself ${ }^{1-13}$. However, EOAD only accounts for a small minority of AD cases. Late onset sporadic AD (LOAD) is more frequent and accounts for up to $99 \%$ or more of AD cases. While increased age is the strongest risk factor and several environmental factors also confer risk for LOAD, its heritability has been estimated to be as high as $79 \%{ }^{14}$.

In contrast to $\mathrm{EOAD}$, genetic risk for $\mathrm{LOAD}$ is less well understood. The $\varepsilon 4$ allele comprising mutations in two codons in Apolipoprotein $\mathrm{E}(A P O E)$ has been identified as the strongest genetic risk factor for LOAD $^{15-19}$. More recently, genome wide association studies (GWAS) $)^{20-27}$ have reproduced the APOE association and also identified 28 other unique loci harboring genetic variants which increase risk for developing LOAD ${ }^{26-28}$. Strikingly, from the set of most significant (or "sentinel") single nucleotide polymorphisms (SNPs) derived from GWAS and SNPs in strong linkage disequilibrium (LD) with them, only $2 \%$ localize in known exons. Since these SNPs do not alter protein sequence, it is difficult to trace their functional importance in disease onset and progression. 
To this end, epigenomic studies are revealing that these SNPs likely alter the function of gene regulatory elements in $\mathrm{AD} .26 \%$ of these SNPs localize in regions containing promoter histone marks, $69 \%$ lie in enhancer states and $46 \%$ lie in DNase I accessible sites ${ }^{26,29}$. Further, previous research shows that the human orthologues of enhancers with increased activity in the CK-p25 mouse model of neurodegeneration overlap with non-coding $\mathrm{AD}$ associated $\mathrm{SNPs}^{30}$. Recently, these SNPs were also found to be primarily contained within microglial enhancers ${ }^{31}$. Furthermore, deregulation of histone 3 lysine 27 acetylation (H3K27ac) and histone 4 lysine 16 acetylation (H4K16ac) was found at loci harboring noncoding $\mathrm{AD}$ associated SNPs in the human postmortem $\mathrm{AD}$ brain $^{32,33}$. Beyond $\mathrm{AD}$ risk loci, changes in histone 3 lysine 9 acetylation (H3K9ac) driven by tau pathology have also been observed in the aging and $\mathrm{AD}$ brain $^{34}$.

Gene regulatory elements, especially enhancers, are highly context-specific with differing activities across tissues, cell types and environments ${ }^{35}$. Therefore, it is likely that different cell types in the brain orchestrate different regulatory programs during $\mathrm{AD}$ progression. Indeed, many LOAD risk loci are primarily implicated in immune function, suggesting differential $\mathrm{AD}$-associated epigenomic mechanisms in immune cell types such as microglia versus neuronal cell types ${ }^{30,36-38}$. Notably, many of the abovementioned studies were performed utilizing whole brain tissue, and not all were performed with tissue from AD patients. Therefore, these epigenomic experiments obscure changes that occur within specific cellular populations.

We address these issues by profiling individual cell types deregulated during AD. We utilize fluorescence-activated nuclei sorting (FANS) ${ }^{39}$ to purify neuronal, microglial and other glial populations in the dorsolateral prefrontal cortex (dlPFC) and hippocampus of subjects with and without AD pathology. Then, we perform chromatin immunoprecipitation and sequencing (ChIP-seq) for H3K27ac, which is associated with active promoters and enhancers ${ }^{40}$, to mark putative regulatory elements (peaks) in these populations.

In addition to establishing the first genome-wide $\mathrm{H} 3 \mathrm{~K} 27 \mathrm{ac}$ profiles in neuronal, microglial, and oligodendrocyte-enriched glial populations from persons with and without AD, our cell type-specific approach confirms enriched $\mathrm{H} 3 \mathrm{~K} 27 \mathrm{ac}$ signatures at GWAS derived LOAD risk loci primarily in microglia. Further, in both the hippocampus and dIPFC, we find strong A $\beta$-associated deregulation of $\mathrm{H} 3 \mathrm{~K} 27 \mathrm{ac}$ in the oligodendrocyte-enriched glial population near AD risk loci and myelin-associated genes. These findings suggest distinct gene-regulatory mechanisms of $\mathrm{AD}$ onset and progression in different brain cell types and highlight specific cell types, loci and pathways for future study.

\section{RESULTS:}

\section{Fluorescence-activated nuclei sorting and H3K27ac ChIP-seq of dIPFC and hippocampus}

We obtained 10 dlPFC and 16 hippocampus samples from participants in either the Religious Orders Study or Rush Memory and Aging Project $(\text { ROSMAP })^{41-43}$ (mean age $=87.84$, s.d. $=7.75$, range $=74.77$ 101.94). 5 of 10 dlPFC samples and 10 of 16 hippocampus samples displayed high $A \beta$ load across the brain, indicative of LOAD (mean percentage area occupied by A $\beta$ across 8 brain regions $=7.30$, s.d = 4.14, range $=2.31-15.40)$ (Supplementary Table 1, Supplementary Figure 1). The brains with A $\beta$ load also displayed high overall neurofibrillary tangle density (mean density of NFT across 8 brain regions $=$ 22.81 , s.d. $=13.73$, range $=1.80-61.01$ ). The self-reported sex of 6 of the 10 dlPFC samples was male, and the remaining 4 were female. Of the 16 hippocampus samples, the self-reported sex of 6 was male, and the remaining 10 were female. 
For each sample, we used FANS to collect NeuN+, Pu.1+, and NeuN-/Pu.1- nuclei to obtain putative neuronal, microglial, and other glial populations, respectively (Figure 1a, Supplementary Figure 2) ${ }^{39}$. On each collected population, we performed ChIP-Seq for H3K27ac, which is associated with transcriptionally active promoters and enhancers ${ }^{40}$. We assessed sample quality by calling regions of H3K27ac enrichment (peaks) for each individual sequencing sample and computing quality metrics based on standard ENCODE guidelines ${ }^{44}$. We detected an average of 91,614 (s.d = 21,197, range=50,662149,681 ) peaks per sample. These peaks overlapped with a large fraction of the sequencing reads (mean $\mathrm{FRiP}=0.256$, s.d. $=0.136$, range $=0.047-0.567$ ), comparable to previous high quality H3K27ac profiles ${ }^{35}$. We curated samples further based on normalized strand cross correlation (NSC) and relative strand cross correlation (RSC) measures to ensure that we retained the highest quality sequencing samples for all downstream analysis (Methods, Supplementary Figure 1).

Then, for each brain region and each cell population, we used ENCODE recommended approaches ${ }^{44}$ to call $\mathrm{H} 3 \mathrm{~K} 27 \mathrm{ac}$ peaks that are reproducible across subjects with $\mathrm{A} \beta$ load, and separately, peaks that are reproducible across subjects without $A \beta$ load. We created a union of all these peak sets representing the combined histone acetylome of the three profiled cell populations in the dIPFC and hippocampus of subjects with and without A $\beta$ load (Supplementary Table 2). We then used DESeq $2^{45}$ to obtain peaks that are significantly hyperacetylated in (i) the NeuN+ population relative to the $\mathrm{Pu} .1+$ and NeuN-/Pu.1populations, (ii) the $\mathrm{Pu} .1+$ population relative to the $\mathrm{NeuN}+$ and $\mathrm{NeuN}-\mathrm{Pu} .1-$ populations, and (iii) the $\mathrm{NeuN}-\mathrm{Pu} .1$ - population relative to $\mathrm{NeuN}+$ and $\mathrm{Pu} .1+$ populations (FDR q<0.05). We performed principal component analysis (PCA) of all samples and observed groupings primarily based on FANS population, with $53 \%$ of the variance explaining the difference between $\mathrm{NeuN}+$ samples and other samples (Supplementary Figure 3).

\section{Active promoters and enhancers in neurons, microglia and oligodendrocyte enriched glia}

As a first step to assess the efficacy of FANS sorting, we generated genome browser tracks of H3K27ac signal for each population by averaging signal across control subjects displaying no A $\beta$. We visualized these genome browser tracks near genes encoding the cell type-specific proteins used to sort out neurons and microglia - RBFOX3 which encodes NeuN, and SPII which encodes Pu.1 (Figure 1b). As expected, we observed average hyperacetylation at the locus containing RBFOX3 in the NeuN+ samples and average hyperacetylation in the Pu.1+ samples at the locus containing SPI1, suggesting successful sorting. Interestingly, we observed hyperacetylation in the NeuN-/Pu.1- samples near genes that are highly expressed in oligodendrocytes, such as $O L I G 2$, suggesting oligodendrocyte enrichment.

To confirm these initial qualitative assessments of sorting efficacy, and to identify the cell types captured in the NeuN-/Pu.1- population, we performed a more rigorous comparison of our H3K27ac ChIP-seq data with an independent higher-resolution single nucleus gene expression (snRNA-seq) dataset from human prefrontal cortex and hippocampus described in Habib et al. ${ }^{46}$. As expected, the NeuN+ samples displayed significant hyperacetylation on average at peaks annotated to nearby genes significantly upregulated in excitatory neuron clusters from prefrontal cortex (adjusted $\mathrm{p}=1.8 \mathrm{e}-204,1.25 \mathrm{e}-92$ ), hippocampus (adjusted $\mathrm{p}=3.37 \mathrm{e}-173,1.20 \mathrm{e}-80$ ), and dentate gyrus (adjusted $\mathrm{p}=2.23 \mathrm{e}-35$ ), and also GABAergic neuron clusters (adjusted p=5.4e-28, 1.4e-31) (Supplementary Figure 4b). Similarly, the Pu.1+ samples displayed significant hyperacetylation on average at peaks annotated to genes significantly upregulated in microglia (adjusted $\mathrm{p}=3.18 \mathrm{e}-22$ ). Strikingly, the NeuN-/Pu.1- samples displayed significant hyperacetylation on average at peaks annotated to genes significantly upregulated in oligodendrocyte clusters (adjusted $\mathrm{p}=1 \mathrm{e}$ $58,1.46 \mathrm{e}-25)$, but not any of the other cell types queried, confirming oligodendrocyte enrichment. 
Since AD pathology, brain region, and sex could potentially influence sample quality and sorting efficacy, we repeated this analysis separately for (i) samples with and without $A \beta$, (ii) samples from dlPFC and hippocampus, (iii) male and female samples, and (iv) each sample individually. In each of these analyses, we observed neuronal enrichment in NeuN+ samples, microglial enrichment in Pu.1+ samples, and oligodendrocyte enrichment in NeuN-/Pu.1- samples (Figure 1c, Supplementary Figure 4c). Since enhancers are known to have long range effects and may not necessarily regulate their nearest genes, we also restricted the analysis to peaks proximal to gene transcription start sites (TSS) $(<5$ kilobases) and observed the same results (Figure 1c, Supplementary Figure 4a). Therefore, we conclude that the NeuN+ population successfully captures neurons, the Pu.1+ population successfully captures microglia, and the NeuN-/Pu.1- population is highly enriched for oligodendrocytes.

Together, our peak annotations represent the first genome-wide maps of H3K27ac in microglia, neurons, and oligodendrocyte-enriched glial (OEG) populations in the human hippocampus and dlPFC of subjects with and without $A \beta$. These annotations enable a better understanding of the gene regulatory roles of the profiled cell types in many different contexts, not limited to AD. Nevertheless, in the next sections, we utilize these annotations to understand cell type-specific epigenomic mechanisms in AD. First, we compare these annotations with GWAS data to annotate LOAD associated SNPs to the cell types and regulatory elements they may potentially disrupt. Second, we perform multiple histone acetylome-wide association studies in each sex, brain region, and cell type to identify AD-associated variations in acetylation. Third, we perform a histone acetylome-wide association study to identify acetylation differences associated with age in each cell type.

\section{GWAS derived common SNPs associated with LOAD risk preferentially colocalize with the microglial histone acetylome}

We performed partitioned heritability analysis by stratified LD score regression ${ }^{47-49}$ (S-LDSC) to estimate the strength of colocalization between $\mathrm{H} 3 \mathrm{~K} 27$ ac peaks that are significantly hyperacetylated on average across subjects in the 3 populations and AD SNP heritability derived from two large AD GWAS meta analyses (Jansen et al. and Kunkle et al.) ${ }^{26,27}$. Strikingly, microglial hyperacetylated peaks displayed a statistically significant preference for colocalization with AD SNP heritability (Figure 2a and b; Jansen et al. GWAS coefficient $=1.6 \mathrm{e}-08, \mathrm{p}=5.28 \mathrm{e}-5$, Kunkle et al. GWAS coefficient $=1.94 \mathrm{e}-08, \mathrm{p}=3.74 \mathrm{e}-3$ ) relative to neuronal and OEG hyperacetylated peaks. Since choice of computational method can influence these assessments, we repeated the analysis with an independent method that utilizes a permutation test ${ }^{35,50}$. We again observed that AD SNP heritability has a strong preference for colocalization with microglial hyperacetylated peaks (Supplementary Figure 5; Kunkle $\log 2 \mathrm{FC}=+0.39$, adjusted $\mathrm{p}=1 \mathrm{e}-06$, Jansen $\log 2 \mathrm{FC}=+0.33$, adjusted $\mathrm{p}=1 \mathrm{e}-06$ ). Further, conducting these analyses with reproducible peaks for each cell type, as opposed to hyperacetylated peaks led to similar results. These findings agree with previous analyses conducted on myeloid cells ${ }^{50-52}$, reinforcing the hypothesis that myeloid cell gene regulation strongly influences predisposition towards $\mathrm{AD}$.

We note that neuronal hyperacetylated peaks overlap with a lower number of GWAS derived AD associated SNPs compared to microglial and OEG hyperacetylated peaks (Supplementary Figure 5; Figure 2c). This finding is consistent with previous analyses conducted on bulk brain tissue maps of histone modifications ${ }^{35,50}$ and open chromatin ${ }^{37,38}$, where signal from neuronal regulatory elements is dominant. Since biases in GWAS sampling and neuronal sample quality could potentially influence the results of these analyses, we used S-LDSC to partition Schizophrenia SNP heritability ${ }^{53}$ across the hyperacetylated peaks in the 3 populations. Only neuronal hyperacetylated peaks displayed significant colocalization (Supplementary Figure 6; coefficient $=1.5 \mathrm{e}-07, \mathrm{p}=1.4 \mathrm{e}-8$ ). This agrees with previous 
findings about Schizophrenia ${ }^{54,55}$, and therefore, confirms that the analysis is robust to biases in GWAS sampling and neuronal sample quality.

\section{Interpreting cell-type specificity and potential disruptions of non-coding AD associated variants}

We annotate non-coding sentinel SNPs identified in Jansen et al. and Kunkle et al. to nearby peaks $(<1 \mathrm{~kb}$ cutoff), enabling assessment of their potential cell type-specific functional effects (Figure 2c, Table 1). As expected, at a majority of GWAS derived risk loci, the sentinel SNPs directly overlap with peaks that are most strongly hyperacetylated in microglia. However, many sentinel SNPs including SNPs at loci containing BIN1, CLU, ADAM10, and CRI directly overlap peaks that are most strongly hyperacetylated in OEG. Only 2 sentinel SNPs overlap with peaks that are most strongly acetylated in neurons. Further, the peaks closest to the TSS of APP and PSENI display the strongest acetylation in OEG, whereas the peak closest to the TSS of PSEN2 display the strongest acetylation in microglia.

Overall, these annotations improve the interpretation of the functional effects of non-coding LOADassociated SNPs. We point out specific examples such as the locus containing the INPP5D gene, where the sentinel SNP rs10933431 (GWAS p-values $=8.9 \mathrm{e}-10,2.5 \mathrm{e}-07)$ overlaps a peak that is acetylated only in microglia but not neurons and OEG ((Figure 2d). Previously, rs10933431 has been shown to overlap with DNase I hypersensitive sites in peripheral blood cells and tissues, including natural killer cells and CD14+ monocytes $^{29}$. Further, rs10933431 disrupts a binding motif for the paired box transcription factor Pax- $5^{29,56}$, which is important for immune cell maturation. Combined, this suggests that rs 10933431 is likely altering regulatory function in immune cell types and microglia, and future studies on the functional effect of this SNP should include culture or model systems that can capture phenotypes of these cell types.

Secondly, at the locus containing the BIN1 gene, which displays the second largest genome wide AD association behind the APOE containing locus, two sentinel SNPs overlap a peak which is acetylated in both microglia and OEG (Figure 2e), but not neurons. One of the SNPs, rs4663105 (GWAS p-values = 3.37e-44, 2.16e-26) has known expression quantitative loci (eQTL) associations with BIN1 gene expression in whole blood and lymphoblastoid cells ${ }^{57,58}$. Similarly, the other SNP, rs6733839 (GWAS pvalues $=1.28 \mathrm{e}-29,4.02 \mathrm{e}-28)$ is a BIN1 eQTL in artery and lymphoblastoid cells ${ }^{57,58}$. Previously, rs6733839 has been shown to overlap with DNase I hypersensitive sites in natural killer cells and CD14+ monocytes ${ }^{29}$. Recently, another study has found that the enhancer overlapping rs6733839 interacts with the BIN1 promoter in microglia ${ }^{31}$. Further, deletion of this enhancer using CRISPR-Cas9 editing altered $B I N 1$ expression in inducible pluripotent stem cell (iPSC) derived microglia, but not neurons and astrocytes. This points towards a role for rs6733839 in disrupting BIN1 expression in cells of the myeloid lineage. However, effects of rs6733839 on BIN1 expression in oligodendrocytes have not been previously studied and therefore, cannot be excluded since the peak is also strongly acetylated in OEG. Further, the other sentinel SNP, rs4663105, could also potentially exert effects on BIN1 expression in microglia or oligodendrocytes, and future studies can help clarify this.

Similarly, at the locus containing PICALM, one sentinel SNP, rs10792832 (GWAS p-values $=7.36 \mathrm{e}-18$, 7.55e-16) and another SNP in tight linkage, rs3851179 (GWAS p-values $=2.02 \mathrm{e}-17,5.81 \mathrm{e}-16$ ) overlap non-neuronal peaks (Figure 2f). This suggests that these SNPs are potentially exerting effects on expression of PICALM in microglia and/or oligodendrocytes, and models of these cell types should be included in future studies to assess their functional effects.

These examples highlight the utility of our data resource in informing future studies of non-coding SNPs associated with traits that include, but are not limited to AD. 


\section{OEG display strongest AD associated acetylation differences}

In each brain region, sex, and cell type, we used a histone acetylome-wide association study to identify acetylation differences between subjects with and without A $\beta$ load using DESeq2 ${ }^{45}$. Overall, we discovered 3598 amyloid-associated differentially acetylated regions (DARs) across all experiments (Supplementary Table 3, Figure 3a).

Unexpectedly, we observed minimal differences in acetylation associated with $A \beta$ load in microglia. In contrast, the OEG population is associated with the largest acetylation differences and contributes to a majority of identified DARs. We discovered two DAR sets, the largest in female hippocampus OEG samples (1962 hypoacetylated; $\mathrm{q}<0.05)$ and the second largest in dIPFC OEG samples $(1029$ hyperacetylated; $\mathrm{q}<0.05)$ that make up $80.3 \%(2,890)$ of the full set of 3,598 DARs. We confirmed that both DAR sets display progressive trends of differential acetylation when treating $A \beta$ load as a continuous variable. Further, in a post-hoc analysis, we controlled for covariates such as age at death and years of education which display no correlation with acetylation levels at these DARs in the corresponding OEG populations (Supplementary Figure 7).

\section{Hypoacetylation in OEG of the hippocampus}

We discovered 1962 hypoacetylated DARs in female hippocampus OEG samples, 81.7\% of which are peaks proximal to TSS $(<5 \mathrm{~kb}$ ) (hypergeometric test $\mathrm{p}$-value=0, Figure 3d), suggesting strong links with promoter activity and gene transcription. Strikingly, this hypoacetylated DAR set includes peaks at the promoters of APP, PSEN1, and PSEN2, the three genes associated with EOAD risk, as well as promoters at several LOAD risk loci identified by GWAS, including BIN1, PICALM, ADAMTS4, ADAM10, and FERMT2 (Supplementary Table 4, Figure 3b, Figure 4a-i, Supplementary Figure 8). Notably, promoters of genes involved in all three secretase complexes including $\alpha$-secretase (ADAM10), $\beta$ secretase (BACE1), and $\gamma$-secretase (PSEN1, PSEN2 and PSENEN) are hypoacetylated, suggesting A $\beta$ processing is directly disrupted in oligodendrocytes.

We performed gene ontology analysis of these DARs using GREAT ${ }^{59}$ which revealed an enrichment for central nervous system myelination, oligodendrocyte development, and oligodendrocyte differentiation (Supplementary Table 5, Figure 3e). We also observed hypoacetylation near genes encoding the five mitochondrial complexes that regulate oxidative phosphorylation (Supplementary Figure 9). Since acetylation differences associated with myelination, oligodendrocyte differentiation and oxidative phosphorylation occurs in tandem with acetylation differences at AD risk genes and amyloid processing, these pathways may directly contribute to $\mathrm{AD}$ onset and progression.

To assess whether these acetylation differences are associated with differences in transcription in oligodendrocytes, we performed quantitative RT-PCR (qRT-PCR) for multiple genes annotated to peaks in this DAR set in oligodendrocyte (Olig2+) nuclei collected from hippocampus samples of a larger set of subjects with and without $A \beta$ from the same cohort (Figure 4j, Supplementary Figure 10). ADAMTS4, PICALM, and FERMT2 displayed significant decreases in transcript levels when comparing low and mid$\mathrm{A} \beta$ load subjects against high $\mathrm{A} \beta$ load subjects. $A P P(\mathrm{p}=0.083), B I N 1(\mathrm{p}=0.157)$, and PSEN1 ( $\mathrm{p}=0.077)$ displayed similar fold decreases that did not meet the $\mathrm{p}$-value cutoff. Transcriptional differences did not display sex-specificity. Combined, this strongly suggests that EOAD and LOAD may share common pathogenic mechanisms in the oligodendrocytes of the human hippocampus.

We discovered the strongest hypoacetylation in this DAR set at a peak annotated to the STMN4 gene $(\log 2 \mathrm{FC}=-1.12$, FDR $\mathrm{q}=1 \mathrm{e}-6)$ which is preferentially expressed in brain tissue ${ }^{60}$ and has known functions in neuron projection development and microtubule polymerization ${ }^{61}$. Notably, several other peaks near 
the STMN4 gene, including a peak at the STMN4 promoter, displayed significant hypoacetylation. MYRF, a transcription factor which directly activates myelination ${ }^{62}$ and has been previously linked to LOAD risk $^{63}$, also displayed strong promoter hypoacetylation ( $\log 2 \mathrm{FC}=-0.48$, FDR q=0.03). STMN4 and MYRF also display significantly reduced transcription in qRT-PCR analysis of oligodendrocyte in subjects with $\mathrm{AD}$. We highlight these myelination-associated genes as high-confidence targets for further investigation in neurodegenerative disorders.

While peaks in this DAR set are annotated to loci associated with AD risk, we did not observe significant colocalization of this DAR set with GWAS derived AD-associated SNPs relative to the full set of peaks active in the profiled cell types and brain regions of AD and non-AD subjects (Jansen coefficient $=3.9 \mathrm{e}-$ 08, $\mathrm{p}=0.198$, Kunkle coefficient=1.11e-07, $\mathrm{p}=0.29$ ). Therefore, SNPs associated with AD from GWAS are unlikely to directly alter the regulatory function of these DARs directly.

\section{Hyperacetylation in OEG of the dIPFC}

We discovered the second largest histone acetylome variation comprising 1029 hyperacetylated DARs in dIPFC oligodendrocyte-enriched glia (OEG) samples. While this DAR set is distinct from the DARs discovered in female hippocampus OEG samples, and contains a lower proportion (60.9\%) of TSS proximal peaks $(<5 \mathrm{~kb})$, we again observed significant hyperacetylation at both EOAD and LOAD risk loci (Supplementary Table 4, Figure 3c, Figure 4a-i, Supplementary Figure 8). This includes four distal intergenic peaks annotated to PSEN2, one distal peak annotated to BIN1, and peaks overlapping the promoters of $C L U, A D A M T S 4$, and SORL1. Furthermore, we observed significant hyperacetylation at three distal peaks annotated to the MAPT gene, which encodes for the tau protein, involved in formation of NFTs.

We performed gene ontology enrichment analysis of this DAR set which again revealed a strong enrichment for central nervous system myelination and oligodendrocyte differentiation (Figure 3e, Supplementary Table 5). In addition, we discovered enrichment for mitochondrion organization, macroautophagy and viral transcription.

We tested whether these acetylation differences are associated with differences in transcription in dIPFC oligodendrocytes by comparing with a previously published snRNA-seq study of dlPFC in $\mathrm{AD}^{64}$. On average, genes annotated to these DARs display higher transcription levels in oligodendrocytes of subjects with $\mathrm{AD}$ (Figure 4k) compared to subjects without AD. Genes associated with LOAD risk including $C L U$ and BINI displayed statistically significant upregulation, while SORL1 (FDR q no vs path. $=0.26$, FDR q no vs early path.=0.70) displayed upregulation that did not meet the q-value cutoff. We note that PSEN2 (FDR q no vs path.=0.18, FDR q no vs early path.=0.21) and MAPT (FDR q no vs path.=0.1, FDR q no vs early path.=0.09) display a downregulation in transcription with AD pathology, which did not meet the q-value cutoff. Hyperacetylated peaks near PSEN2 and MAPT are distal to the TSS, and therefore, are probably enhancer peaks. Enhancers have been known to regulate target gene expression over long distances and hence, effects on distal transcripts cannot be excluded.

We observed the strongest hyperacetylation at a peak near the promoter of the ADAMTS18 gene $(\log 2 \mathrm{FC}=3.4, \mathrm{FDR} \mathrm{q}=5.1 \mathrm{e}-81)$, which is a member of the ADAMTS family of metalloproteinases with thrombospondin motifs. This family of proteins is known to play a role in neuroplasticity and has been widely studied for its role in $\mathrm{AD}^{65}$. Overall, 38 different peaks annotated to the ADAMTS18 gene displayed significant hyperacetylation across DAR sets specific to dIPFC microglia and oligodendrocyteenriched glia, but not neurons. ADAMTS18 also displayed statistically significant increase in transcription in dIPFC oligodendrocytes of AD subjects. These results suggest that ADAMTS18 gene regulation is 
heavily altered in $\mathrm{AD}$ in the dlPFC and resides in an important oligodendrocyte and microglia-specific locus that requires further study.

Similar to the female hippocampus OEG hypoacetylated DAR set, we did not observe significant colocalization between GWAS derived AD-associated SNPs and peaks in this DAR set using S-LDSC (Jansen GWAS coefficient =-2.57e-08, p=0.70, Kunkle GWAS coefficient=-1.26e-07, p=0.74).

Therefore, SNPs associated with AD from GWAS are unlikely to alter the regulatory function of these DARs directly.

Overall, we reveal that common pathways associated with both early and late onset AD are likely perturbed at the epigenomic level in oligodendrocyte-enriched glia. We show that amyloid processing, central nervous system myelination and oligodendrocyte processes are significantly altered in dlPFC and hippocampus of subjects with amyloid pathology and display acetylation differences in tandem with AD risk genes. We also highlight novel genomic loci that display large changes in acetylation in glia in AD including ADAMTS18, STMN4 and MYRF. Taken together, the sets of DARs we have described are strong candidate targets for AD therapeutics in oligodendrocytes that utilize technologies such as CRISPR-Cas9 genome editing ${ }^{66}$.

We also performed unsupervised clustering of the full set of 3,598 DARs to identify modules that display correlated acetylation across the profiled cell type populations and brain regions. We separately clustered TSS proximal and TSS distal DARs to identify putative promoter and putative enhancer modules, respectively. Modules of both proximal and distal peaks tended to separate based on cell type-specificity. We note that majority of peaks display microglia or OEG specificity (Supplementary Figure 11) with little or no acetylation in neuronal samples. This highlights the utility of our data resource in identifying novel gene regulatory modules that are cell type-specific and associated with disease.

\section{Age associated acetylation differences are enriched in the microglial population}

While microglial H3K27ac displays strong colocalization with GWAS derived AD associated SNPs, unexpectedly, the microglial population displays very few acetylation differences associated with A $\beta$ load. Contrastingly, the microglial population displays the strongest age-associated acetylation differences encompassing both dIPFC and hippocampus, in an analysis that controlled for A $\beta$ load, sex, and brain region differences (Supplementary Figure 12). We identified 391 peaks that are significantly hypoacetylated with increasing age and 53 peaks that are significantly hyperacetylated with increasing age (FDR q < 0.05) (Supplementary Table 6). We mapped these peaks using GREAT ${ }^{59}$ and discovered 2 hypoacetylated peaks annotated to the amyloid precursor protein $(A P P)$ gene, and 6 hypoacetylated peaks near the LRRTM3 gene, which is involved in positive regulation of A $\beta$ formation (Supplementary Table 7). This suggests that $A \beta$ processing may be altered in microglia with increasing age. We also observed hyperacetylation at 3 distal peaks annotated to the FKBP4 gene, which is involved in tau protein binding and influences neurofibrillary tangle formation. Although further investigation is required, these findings point towards a role for age-associated epigenomic changes in microglia influencing the onset and progression of LOAD.

\section{DISCUSSION:}

We report the first $\mathrm{H} 3 \mathrm{~K} 27 \mathrm{ac}$ maps for sorted neurons, microglia, and oligodendrocyte-enriched glia from both the hippocampus and dIPFC of postmortem human brain tissue. We find microglial H3K27ac peaks have a stronger preference for colocalization with common SNPs associated with LOAD risk relative to the other neural cell types profiled, supporting previous findings ${ }^{30,31,37,38}$. While this suggests a significant causal role for LOAD risk loci influencing AD predisposition and progression through microglial 
processes, perhaps unexpectedly, comparison of $\mathrm{H} 3 \mathrm{~K} 27$ ac peaks by AD diagnosis in microglia revealed few differences. Instead, we report $\mathrm{H} 3 \mathrm{~K} 27 \mathrm{ac}$ is altered significantly with age in microglia, leading us to conclude that amongst the individuals analyzed, microglial $\mathrm{H} 3 \mathrm{~K} 27 \mathrm{ac}$ is more responsive to advances in age than to $A \beta$ load. We note that heterogeneity within the microglial population in disease has been previously reported ${ }^{52,67}$ and therefore, the possibility of $\mathrm{AD}$ associated gene regulatory differences in microglia cannot be excluded based on our study which profiles the microglial population in bulk, and hence, represents average microglial signal. However, recent single cell transcriptome profiling of microglia in $\mathrm{AD}$ subjects revealed no differences in both the composition of microglial subpopulations as well as gene expression, supporting the findings from our study ${ }^{68}$.

Beyond microglia, we also find a subset of AD risk loci have significant $\mathrm{H} 3 \mathrm{~K} 27 \mathrm{ac}$ signal in oligodendrocyte-enriched glia relative to other cell types. These include risk loci associated with genes $C L U, B I N 1$, and PICALM. Additionally, the transcriptional start sites of EOAD genes APP and PSENI also show significant $\mathrm{H} 3 \mathrm{~K} 27$ ac enrichment in oligodendrocytes relative to other cell types. Previous multi-scale network analyses have found oligodendrocyte transcript and protein modules are enriched for genes associated with AD risk loci, particularly BIN1 and PICALM ${ }^{69,70}$. Indeed, BIN1 is highly expressed in oligodendrocytes, and is associated with white matter tracts in the human brain ${ }^{71}$. Combined, these data suggest epigenomic mechanisms in oligodendrocytes play a significant role in the functionality of certain $\mathrm{AD}$ risk loci and their associated risk genes ${ }^{72}$.

In parallel, we also find oligodendrocyte-enriched glia show by far the largest acetylation differences associated with $A \beta$ load. In the hippocampus, the promoters of genes associated with early and late-onset AD risk displayed hypoacetylation. This includes EOAD risk genes APP, PSEN1, and PSEN2, and several genes associated with LOAD risk, including BIN1, PICALM, ADAM10, ADAMTS4, FERMT2, and SORL $1^{25-27,73}$. Sorted hippocampal oligodendrocyte nuclei from an independent cohort of ROSMAP individuals were used to assess transcript levels of these genes, which revealed a corresponding downregulation of transcripts in individuals with high $A \beta$ load. This suggests that EOAD and LOAD may share common pathogenic mechanisms in oligodendrocytes. In addition to risk genes, H3K27ac peaks associated with core oligodendrocyte processes such as myelination were significantly hypoacetylated in the hippocampus of AD subjects. Myelin-associated genes STMN4 and MYRF were confirmed to have corresponding transcriptional downregulation in the same independent cohort of ROSMAP individuals.

The hippocampus is one of the earliest brain regions affected by AD pathology ${ }^{74}$. Here, we describe the first cell type-specific $\mathrm{H} 3 \mathrm{~K} 27 \mathrm{ac}$ dataset from the hippocampus of postmortem AD patients. This provides a resource by which we can understand the epigenomic signatures of distinct cell types at a crucial anatomical locus of neurodegeneration. Specifically, it is evident that the H3K27ac changes in oligodendrocyte-enriched glia provide insight as to how the epigenomic state of the hippocampus is altered in AD. White matter lesions are positively correlated with hippocampal atrophy, and white matter hyperintensities are thought to be a core feature of $\mathrm{AD}^{75,76}$. Thus, the marked hypoacetylation observed near genes associated with $A \beta$ processing and myelination in hippocampal oligodendrocytes suggest these biological processes are defective and may directly contribute to AD progression. Importantly, previous $\mathrm{AD}$ studies demonstrate similar pathways are deregulated at the transcriptomic and proteomic levels in oligodendrocyte-enriched modules, as does a recent single-cell gene expression study ${ }^{64,69,70}$. Combined with our current findings, this strongly suggests oligodendrocytes play an active role in AD progression and merit further attention. Although we identified these hypoacetylated peaks from female AD patients, the lack of sex-specificity observed in supporting publications and in our RT-qPCR validation lead us to conclude these DARs most strongly reflect epigenomic changes associated with A $\beta$ load. 
Interestingly, our dataset reveals dIPFC and hippocampus oligodendrocyte-enriched populations mount distinct epigenomic signatures in response to AD. Similar to our findings in the hippocampus, we observed an A $\beta$-correlated deregulation of myelin-associated promoters and enhancers in dlPFC oligodendrocytes. However, these dIPFC DARs become hyperacetylated in AD individuals, as do peaks annotated to PSEN2, CLU, ADAMTS4, BIN1, and SORL1. The DARs in the hippocampus are largely distinct from the DARs in the dIPFC, indicating brain region-specific epigenomic alterations. This disparity between brain regions may reflect oligodendrocyte heterogeneity in response to pathological insults, as well as region-specific differences in cell composition and pathologic severity. Alternatively, it may be associated with compensatory signaling in the prefrontal cortex that has been previously reported in neurodegenerative disorders ${ }^{77}$. However, in total, it is apparent oligodendrocyte H3K27ac represents a core feature of epigenomic dysregulation in both hippocampus and dlPFC.

Many lines of evidence have revealed roles for oligodendrocyte-driven myelination processes in both multiple sclerosis and major depression ${ }^{78-80}$, and studies are ongoing to advance the understanding of glial cells in neurological disorders ${ }^{81}$. The connection between $\mathrm{AD}$ and oligodendrocyte epigenomic dysregulation is not well-understood, and our data highlight this topic as a priority for future research. We propose further investigation into the role of myelination and demyelination is warranted in AD.

Our full set of DARs constitutes a larger list of novel genomic targets related to oligodendrocyte function, $\mathrm{A} \beta$ processing and oxidative phosphorylation that may be targeted using genome editing technologies such as CRISPR-Cas $9^{66}$. For example, the promoter of the disintegrin and metalloprotease ADAMTS18 displays the strongest hyperacetylation in the dlPFC of AD subjects, revealing it as a strong candidate for future therapeutics.

Lastly, we foresee that single nucleus level epigenomic assays for transposase accessible chromatin (snATAC-seq) can enable understanding of disease associated epigenomic deregulation and cell type heterogeneity in disease at a resolution that supersedes our study and previous studies. However, while active enhancers and promoters commonly lie in accessible chromatin regions, H3K27ac is a more robust indicator of active gene regulation, and therefore, our data resource can augment such future studies.

Taken together, our study shows the power of cell type-specific epigenomic profiling in identifying pathways and genomic loci that are differentially regulated in AD. We reveal new cell type-specific processes involved in $\mathrm{AD}$ which opens opportunities to ameliorate its harmful effects by targeting therapeutics to oligodendrocytes. 


\section{METHODS:}

\section{Source of Brain Tissue and Pathologic Data}

Biospecimens and data came from autopsied participants in one of two prospective clinical-pathologic cohort studies, the Religious Orders Study or Rush Memory and Aging Project (ROSMAP). Both studies were approved by an Institutional Review Board of Rush University Medical Center. All participants signed an informed consent, an Anatomical Gift Act, and a repository consent to all their data and biospecimens to be repurposed. Details of the studies have been previously reported ${ }^{43}$.

\section{Fluorescence-Activated Nuclei Sorting}

Frozen dorsolateral prefrontal cortex and hippocampus samples were retrieved from $-80^{\circ} \mathrm{C}$ storage and thawed on ice, then disrupted with a handheld homogenizer. Samples were fixed with $1 \%$ paraformaldehyde for 10 minutes at room temperature. Fixation was quenched with glycine for 5 minutes. Nuclei were isolated by dounce-homogenization followed by filtration through a 70uM cell strainer (cat no. 21008-952, VWR, Radnor PA). To immunotag cell type specific nuclei, anti-NeuN antibody conjugated to Alexa Fluor 488 (cat no. MAB377X, EMD Millipore, Burlington MA), and anti-PU.1 antibody conjugated to Alexa 647 (cat no. 2240S, Cell Signaling Technology, Danvers MA) were incubated with nuclei at $4^{\circ} \mathrm{C}$ for one hour and overnight, respectively. Samples were strained through a 40um filter (21008-949, VWR) and stained with DAPI (D9542, Sigma Aldrich, St. Louis MO) before flow cytometry. Fluorescence activated nuclei sorting was performed until at least 400,000 nuclei were collected for each cell type (NeuN+, Pu.1+, and NeuN-/Pu.1-) using the FACSAria (BD Biosciences, US).

\section{Chromatin Immunoprecipitation}

Following sorting, chromatin was fragmented into 200-600 bp fragments using the Diagenode bioruptor. Fragmented samples were split equally into two tubes such that each tube contained an equivalent of chromatin from 200,000 nuclei. All ChIPs were carried out in duplicate. Samples were pre-cleared with BSA-blocked Protein A sepharose beads (cat no. GE17-0780-01, Sigma Aldrich) for four hours at $4^{\circ} \mathrm{C}$. At this point, $1 \%$ input was collected and stored at $-20^{\circ} \mathrm{C}$. Chromatin was incubated with $2 \mathrm{ug}$ of Histone H3 (acetyl K27) antibody (cat no. ab4729, abcam, Cambridge UK) overnight at $4^{\circ} \mathrm{C}$. Chromatin fragments bound to the antibody were pulled down with BSA-blocked Protein A sepharose beads for four hours at $4^{\circ} \mathrm{C}$. To reduce non-specific binding, the bead-chromatin complex was washed four times with ice-cold RIPA buffer. Immunotagged chromatin was eluted from beads through shaking at $65^{\circ} \mathrm{C}$ for 15 minutes. Both $1 \%$ input and ChIP were de-crosslinked overnight in $\mathrm{T}_{50} \mathrm{E}_{10} \mathrm{~S}_{1}$ buffer at $65^{\circ} \mathrm{C}$. Reverse crosslinked chromatin was treated with RNase A and Proteinase K. DNA was purified using phenolchloroform extraction. Following ethanol precipitation, samples were resuspended in $10 \mathrm{mM}$ Tris-HCl buffer and stored at $-20^{\circ} \mathrm{C}$.

\section{ChIP-seq high-throughput sequencing}

A portion of the sample was used to assess enrichment for cell-type specific H3K27ac peaks via qPCR. If the sample passed qPCR quality control, libraries were generated from the remaining sample. Library generation was performed using the KAPA Hyper Prep Kit (KK8504, Kapa Biosystems). After amplification and quantification, a portion of the library was used for a second qPCR to ensure enrichment of cell-type specific H3K27ac peaks. If the sample passed the second qPCR quality control, it was submitted to the MIT BioMicro Center for fragment analysis, followed by sequencing. The 40-bp 
single-end sequencing was performed using the Illumina HiSeq2000 platform according to standard operating procedures.

\section{Peak Calling, Quality Control and Read Counting}

For peak calling, the AQUAS ChIP-Seq workflow (https://github.com/kundajelab/chipseq_pipeline) was used. To perform quality control, the two technical replicates for each sample were individually input to the AQUAS workflow to compute standard ENCODE quality metrics ${ }^{44}$ such as NSC, RSC, NRF, PBC1, PBC2, FRiP, replicate consistency etc. All samples that did not meet quality standards of (NSC $>1.01$, $\mathrm{RSC}>0.4, \mathrm{PBC} 1>0.4$ ) were discarded at this point. The workflow uses Burrows-Wheeler alignment ${ }^{82}$, Samtools ${ }^{83}$ for processing alignments, MACS $2^{84}$ for peak calling, and PICARD (http://broadinstitute.github.io/picard/) for removing PCR duplicates. Peak reproducibility is assessed by overlapping peaks across groups of sample replicates and pseudoreplicates using a method similar to irreproducible discovery rate (IDR) ${ }^{85}$ analysis. All analysis was performed on the hg19 reference genome.

Reproducible peaks were called on samples pooled by each separate group of samples defined by brain region, cell type population and presence or absence of $A \beta$ load. The mergeBed ${ }^{86}$ utility was then used to merge the set of peaks across these pools. At this step, peaks that were less than $200 \mathrm{bp}$ apart were merged together to account for local depletions in chromatin intensity profiles ("dips") ${ }^{87}$. We propose this merged peak set as a reference set for peaks active in different brain cell types in the dIPFC of AD and non-AD subjects and use it in downstream analyses. The featureCounts ${ }^{88}$ package was used to count the read signal at these peaks for every ChIP-Seq experiment. This read count matrix was then used in downstream analysis for validation of sorting and for identifying differentially acetylated regions using DESeq $2^{45}$. ROSMAP subject metadata were used as post-hoc covariates in the analysis.

\section{Cell type peak sets}

We also generated reproducible peak sets for each cell type by assessing reproducibility across the two brain regions for subjects without $A \beta$ load. This peak set was used to generate the browser visualization tracks at the loci containing the INPP5D, BIN1 and PICALM genes (Figure 2d, e and f). Browser tracks for INPP5D, BINI and PICALM were generated using the integrative genomics viewer (IGV) ${ }^{89}$ and pygenometracks ${ }^{90}$, and edited later.

Further, for each of the three cell type populations, we used the negative binomial model of DESeq $2^{45}$ to identify subsets of differentially hyperacetylated peaks in the focal cell type population against the two non-focal populations from the full set of brain peaks (see Peak Calling, QC and Read Counting). Peaks were defined as differentially hyperacetylated if they displayed a positive log fold change and passed an adjusted p-value threshold of 0.05 . A cell type background peak set was then created from these three sets of peaks using the mergeBed utility. This set of peaks was used in heritability enrichment analyses using permutation testing ${ }^{35,50}$ and stratified LD-score regression ${ }^{47-49}$.

\section{Sorting validation and identification of cell types by comparison to single nucleus RNA-seq clusters}

The full set of merged peaks were annotated to their nearest genes using the annotatePeaks tool in HOMER $^{91}$. Marker gene sets for 15 single nucleus RNA-Seq cell type clusters were downloaded from Habib et $\mathrm{al}^{46}$. For each single nucleus RNA-seq cluster, the set of H3K27ac peaks for which the closest gene was present in the marker gene set was obtained. Then, DESeq $2^{45}$ was used to compute $\log 2 \mathrm{FC}$ at these peaks between ChIP-Seq samples corresponding to a focal foreground cell type population against ChIP-seq samples corresponding to the other two background cell type populations. A one-sided t-test was used to test whether the distribution of $\log 2 \mathrm{FC}$ was significantly greater than 0.5 ( 1.4 fold change). 
A significant result from this test indicated the enrichment of a cell type in the focal ChIP-Seq population. The test was conducted for every pair of focal ChIP-Seq population and single nucleus RNA-Seq cluster. p-values were adjusted for multiple hypothesis testing using Bonferroni's correction.

In addition, a similar approach was used to verify these results at the individual sample level as well as groups of samples defined by $\mathrm{A} \beta$ load, sex and brain region. Variance stabilized counts were used and mean $\log 2 \mathrm{FC}$ for each focal population was computed against the other two non-focal populations. For each of the 15 cell type clusters, the mean $\log 2 \mathrm{FC}$ was then computed for peaks annotated to that cluster and the resulting values were plotted in a heatmap.

To test whether distant peaks confound these results, the above analyses were also conducted on peaks that are near promoters of the marker genes by only considering the peaks that are less than 5 kilobases away from transcription start sites of the 15 gene sets.

\section{Enrichment test for colocalization of AD-associated variants with cell type-specific peaks}

GWAS summary statistics from two studies, Kunkle et $\mathrm{al}^{26}$ and Jansen et $\mathrm{al}^{27}$ were downloaded. and stratified LD-score regression (S-LDSC) ${ }^{47-49}$ was used to compute AD SNP heritability in enrichment in differential peaks for each cell type against the merged background set. The standard workflow described by the authors was used and LD scores were computed based on custom annotations derived from hyperacetylated peaks called on each cell type and compared against custom annotations derived from the merged background set constructed from the three cell type hyperacetylated peak sets. The regression coefficients for each population were extracted and plotted. A significant result from this test indicates an enrichment of genetic risk for $\mathrm{LOAD}$ in regions that are actively regulating gene expression in the cell type, suggesting a role for that cell type in influencing predisposition towards LOAD.

To test whether choice of computational method may confound these results, we used another approach that utilizes a permutation test ${ }^{35,50}$. LD-pruning was applied (LD > 0.5) on both GWAS datasets based on the 1000 genomes reference ${ }^{92}$. SNPs overlapping protein coding sequence ${ }^{93}$ were filtered out along with SNPs in tight linkage disequilibrium (LD > 0.5). SNPs with p-values less than 1e-3 were selected and overlap annotations were created for each set of differential cell type-specific peaks (see Cell type peak sets). A permutation test was used to compute heritability enrichment of AD-associated SNPs in a focal foreground set of differential peaks for a cell type against the merged background set. SNPs were permuted 1,000,000 times preserving distance to gene, minor allele frequency and the number of variants that are in LD.

\section{DARs associated with A $\beta$ load}

Differentially acetylated regions were identified using the negative binomial model of DESeq2 on the previously generated count matrix (see Peak Calling, QC and Read Counting) selecting peaks associated with a binary $A \beta$ load indicator. An adjusted p-value cutoff of 0.05 was used for selecting differentially acetylated peaks. For each differential acetylation model setting (Supplementary Table 3), a reduced count matrix was generated that includes only the subset of samples corresponding to the variables described. Variance stabilized (vst) read counts ${ }^{45}$ across all peaks were used for heatmap visualization and principal components analysis (PCA). Box plots of read counts against covariate variables such as $A \beta$, age, years of education and sample quality metrics were produced using peak-wise Z-scores of vst normalized counts. DAR sets were annotated to their nearest genes using the annotatePeaks tool in HOMER ${ }^{91}$ and the distribution of distance to TSS was plotted for the two biggest DAR sets as well as the remaining DARs. 
Genome browser visualizations were created for the two biggest DAR sets at known EOAD and LOAD risk loci, as well as highly differentially acetylated loci using pygenometracks ${ }^{90}$. Custom UNIX commands and the UCSC bigWigMerge ${ }^{94}$ tool were used to create average H3K27ac signal tracks in oligodendrocyte enriched glia samples of subjects with and without A $\beta$ load. Tracks for DESeq2 $\log 2 \mathrm{FC}$ and UCSC known gene annotations ${ }^{95}$ were included. Generated visualizations were edited later.

The Genomic Region Enrichment and Annotation Tool (GREAT) ${ }^{59}$ web tool was used for computing enrichments for ontological annotations associated with genes proximal to DAR sets. GREAT analysis was performed separately on the two biggest DAR sets as well as the remaining DARs not in those sets. In addition, we used GREAT to annotate neuron, microglia and oligodendrocyte enriched glial hyperacetylated peaks for enriched functions. The merged brain peak set (see Peak Calling, QC and Read Counting) was used as the background for each GREAT analysis. A heatmap of the fold enrichment returned by GREAT was plotted for any GO Biological Process that passed a q-value cutoff of 0.05 and was associated with a minimum of 5 genes in any of the 6 GREAT analyses. In addition, fold enrichment for the KEGG Alzheimer's Disease Pathway was plotted in the heatmap.

S-LDSC was used to test for AD SNP heritability enrichment from both AD GWAS studies in the two biggest DAR sets. The full brain peak set was used as background.

\section{Correlation based clustering of DARs}

Variance stabilized (vst) counts for all $\mathrm{A} \beta$ associated DARs were obtained. All samples passing quality control were included. Clustering was performed separately for TSS proximal $(<5 \mathrm{~kb})$ DARs and TSS distal ( $>5 \mathrm{~kb}$ ) DARs. A distance matrix based on Pearson correlation was computed between every pair of peaks. More specifically, the distance between peak $x$ and peak $y$ was calculated as $1-a b s(\operatorname{cor}(x, y))$, where $a b s$ represents absolute value and cor represents Pearson correlation. The absolute value was used because it gives equal weightage to negatively correlated peaks and positively correlated peaks. Then, average linkage hierarchical clustering using the hclust $\mathrm{R}$ function was performed using this distance matrix to construct a dendrogram. To identify stable clusters from the resulting dendrogram, the a dynamic tree cutting approach ${ }^{96}$ was used. The resulting cluster identities were plotted alongside a heatmap of variance stabilized counts.

\section{RNA extraction, reverse transcription and quantitative PCR in postmortem hippocampus (qPCR)}

An independent set of hippocampal samples from the ROSMAP cohort were used for rt-qPCR validation. Samples were prepped for FANS as described previously. To isolate oligodendrocyte, microglia, astrocyte, and neuronal nuclei, samples were stained overnight at $4{ }^{\circ} \mathrm{C}$ with anti-Olig2 antibody conjugated to Alexa Fluor 488 (cat no. MABN50A4, EMD Millipore, Burlington MA), anti-PU.1 antibody conjugated to Alexa Fluor 647 (cat no. 2240S, Cell Signaling Technology, Danvers MA), anti-GFAP conjugated to Alexa Fluor 555 (cat no. 3656, Cell Signaling Technology, Danvers MA), and stained for one hour with anti-NeuN conjugated to biotin (cat no. MAB377B, EMD Millipore, Burlington MA), and for one hour with Brilliant Violet 711 Streptavadin (cat no. 405241, BioLegend, San Diego, CA). Fluorescence activated nuclei was performed until at least 100,000 Olig2-positive nuclei, NeuN-positive nuclei, GFAP-positive nuclei, and PU.1-positive nuclei were collected for each sample.

Following sorting, nuclei were treated for 15 minutes with Proteinase $\mathrm{K}$ at $50^{\circ} \mathrm{C}$ and then for 13 minutes at $80^{\circ} \mathrm{C}$. RNA was extracted using Direct-zol RNA MicroPrep kit (Zymo Research) according to manufacturer's instructions. Reverse transcription of RNA was carried out using Invitrogen SuperScript IV First Strand Synthesis System (Oligo dT) according to manufacturer's protocol. qPCR was performed using a Bio-Rad CFX-96 quantitative thermocycler and SsoFast EvaGreen Supermix (Bio-Rad). Relative changes in gene expression were determined using the $2^{-\Delta \Delta \mathrm{Ct}}$ method. The geometric mean of cycle numbers from 
RPL13, CYC1, and GADPH were used for housekeeping Ct values. Primer sequences used for qPCR can be found in Supplementary Table 6.

\section{Comparison with snRNA-seq from postmortem dIPFC}

Hyperacetylated DARs in oligodendrocyte enriched glia of the dlPFC were assessed for nearby transcriptional differences identified in the snRNA-seq study from Mathys, Valderrain et $\mathrm{al}^{64}$. The nearest genes of the hyperacetylated DARs were obtained using annotatePeaks in HOMER. The oligodendrocyte cluster specific $\log 2 \mathrm{FC}$ of these genes was obtained from the snRNA-seq study. Then, a one-sample onesided t-test was used to test whether there is an average increase in transcription at these genes (null hypothesis $\log 2 \mathrm{FC}=0$, alternative hypothesis $\log 2 \mathrm{FC}>0$ ). Transcriptional fold-change of specific AD risk genes and genes near highly hyperacetylated peaks was also plotted.

\section{DARs associated with Age}

Age associated changes in H3K27ac levels were identified using DESeq2. To control for potential confounds, sex, the binary A $\beta$ load status, and brain region were added as covariates in the linear model along with age. An adjusted p-value cutoff of 0.05 was then used to screen for peaks differentially acetylated with every unit increase in age. The peaks were grouped as having increased or decreased acetylation with age, and ontological annotation enrichments were computed using GREAT (see DARs associated with A $\boldsymbol{\beta}$ load) using a full brain background peak set. For heatmap visualization, variance stabilizing transformation (vst) was applied on the full matrix and differential peaks were extracted. 


\section{DATA AVAILABILITY:}

The ChIP-seq data will be made available on The Rush Alzheimer's Disease Center (RADC) Research Resource Sharing Hub at https://www.radc.rush.edu/docs/omics.htm or at Synapse (link to be provided) under a doi. The ROSMAP metadata will be accessible at (link to be provided). The data will be available under controlled use conditions set by human privacy regulations. To access the data, a data use agreement will be needed. This registration is in place solely to ensure anonymity of the ROSMAP study participants. A data use agreement will be agreed with either Rush University Medical Center (RUMC) or with SAGE, who maintains Synapse, and will be downloadable from their websites. Bed, narrowPeak and bigwig files that do not contain private information will be made available at the appropriate resource in accordance with privacy considerations.

\section{CODE AVAILABILITY:}

Code for processing and analyzing the data will be made available at:

https://github.com/pfenninglab/ad h3k27ac 3ct

\section{AUTHOR INFORMATION:}

These authors contributed equally: Easwaran Ramamurthy and Gwyneth Welch

These authors jointly supervised the work: Li-Huei Tsai and Andreas Pfenning

\section{CONTRIBUTIONS:}

J.C., G.W., E.R., D.A.B., A.P. and L.-H.T., all contributed to study design. The study was coordinated and directed by A.P. and L.-H.T. J.C. performed FANS and ChIP-seq for dorsolateral prefrontal cortex samples. G.W. performed FANS, ChIP-seq and RT-qPCR for hippocampus samples. E.R. performed ChIP-seq processing and led the computational analyses. Y.Y. did the aging analysis supervised by A.P. and E.R. L.G. performed initial genetics integration analysis supervised by A.P.. E.R., G.W., J.C., Y.Y., D.A.B, L-H.T., and A.P wrote and edited the manuscript.

\section{ACKNOWLEDGEMENTS:}

We thank the study participants and staff of the Rush Alzheimer's Disease Center. This work was supported in part by the Cure Alzheimer's Fund (CAF) and The Okawa Foundation for Information and Telecommunications (A.P.); NIH grants RO1NS102730, RO1AG054012, and The Glenn Foundation for Aging Research (L.-H.T.). E.R was supported by a Presidential fellowship from Carnegie Mellon Brainhub. G.W. was supported by the Barbara Weedon Champions of the Brain Fellowship from MIT Brain and Cognitive Sciences department.

\section{ETHICS DECLARATION:}

A.P served as a paid consultant for Cognition Therapeutics, Inc during preparation of the manuscript. 


\section{FIGURES:}

a
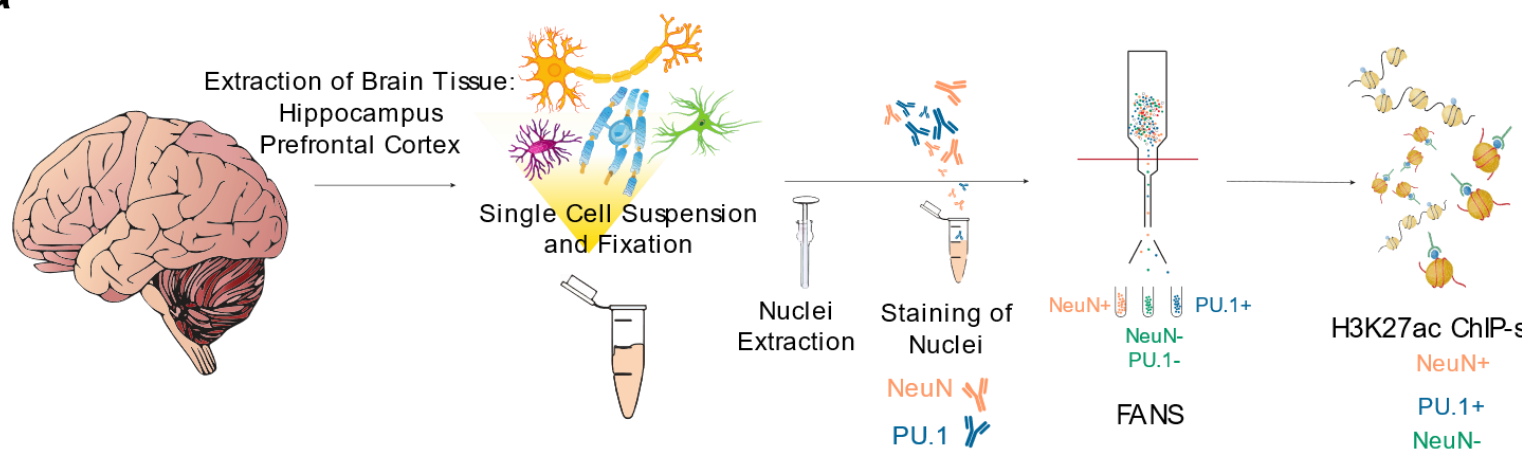

H3K27ac ChIP-seq

Neun+

PU.1+ Neun-

b

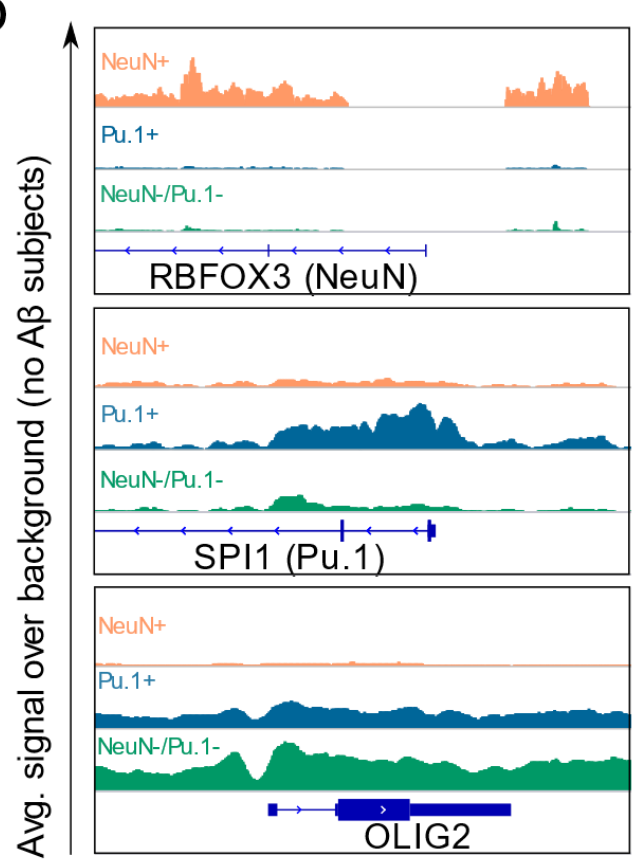

C

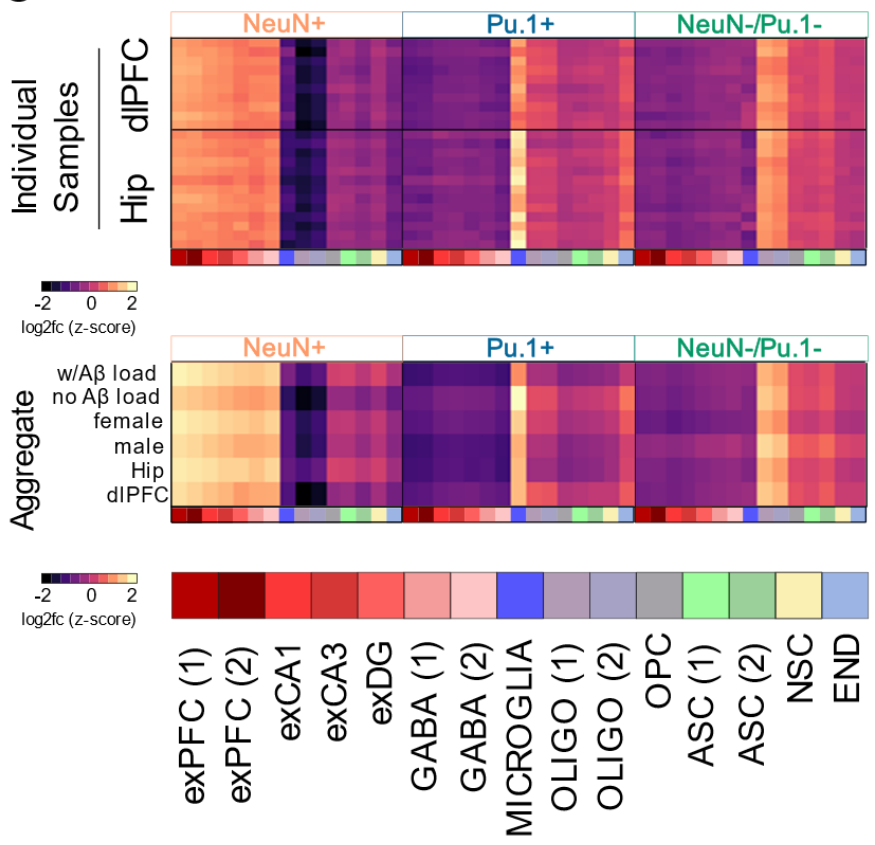

Figure 1: FANS sorting captures neurons, microglia and oligodendrocyte enriched populations from postmortem brain tissue a. Workflow for sorting nuclei and performing H3K27ac ChIP-seq from postmortem human brain tissue b. H3K27ac signal over background (Input) averaged across subjects without A $\beta$ load for each of the three populations near RBFOX3 (NeuN), SPI1 (Pu.1) and OLIG2 (an oligodendrocyte marker) c. Top. for every individual hippocampus and dlPFC tissue sample, fold-change ( $\log 2$ transformed) of H3K27ac signal in each focal cell population over the other two non-focal cell populations, averaged across peaks near the promoters ( $<5 \mathrm{~kb}$ from transcription start site) of genes defined to be markers for 15 different cell types in Habib et $\mathrm{al}^{46}$. bottom. collapsed versions of top heatmap representing averages across subjects defined by different stratifications of $A \beta$ load, sex and brain region. Abbreviated labels for the single nucleus analysis clusters are presented. exPFC, glutamatergic neurons from the PFC; GABA, GABAergic interneurons; exCA1/3, pyramidal neurons from the hippocampus $\mathrm{CA}$ region; exDG, granule neurons from the hippocampus dentate gyrus region; ASC, astrocytes; MICROGLIA, microglia; OLIGO, oligodendrocytes; OPC, oligodendrocyte precursor cells; NSC, neuronal stem cells; END, endothelial cells. 
a

b
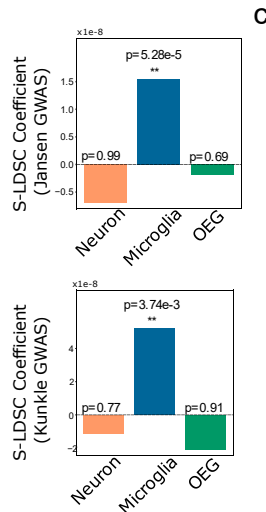

d

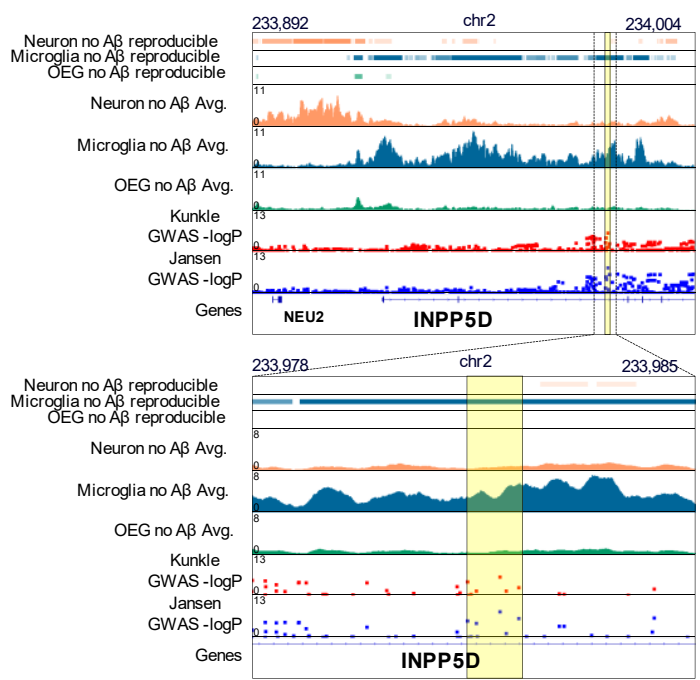

Kunkle GWAS Sentinel SNPs
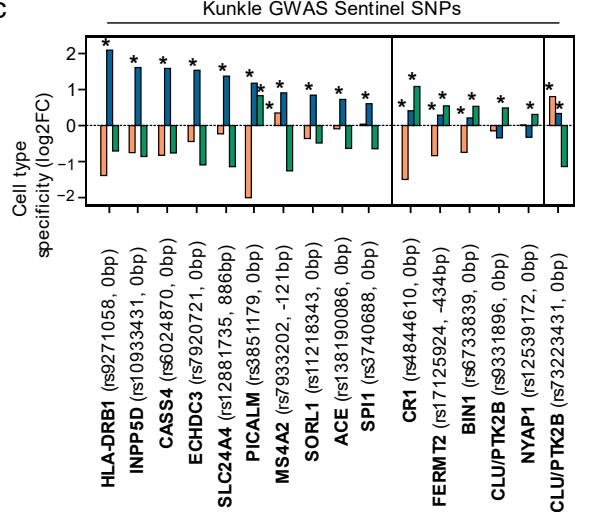

e
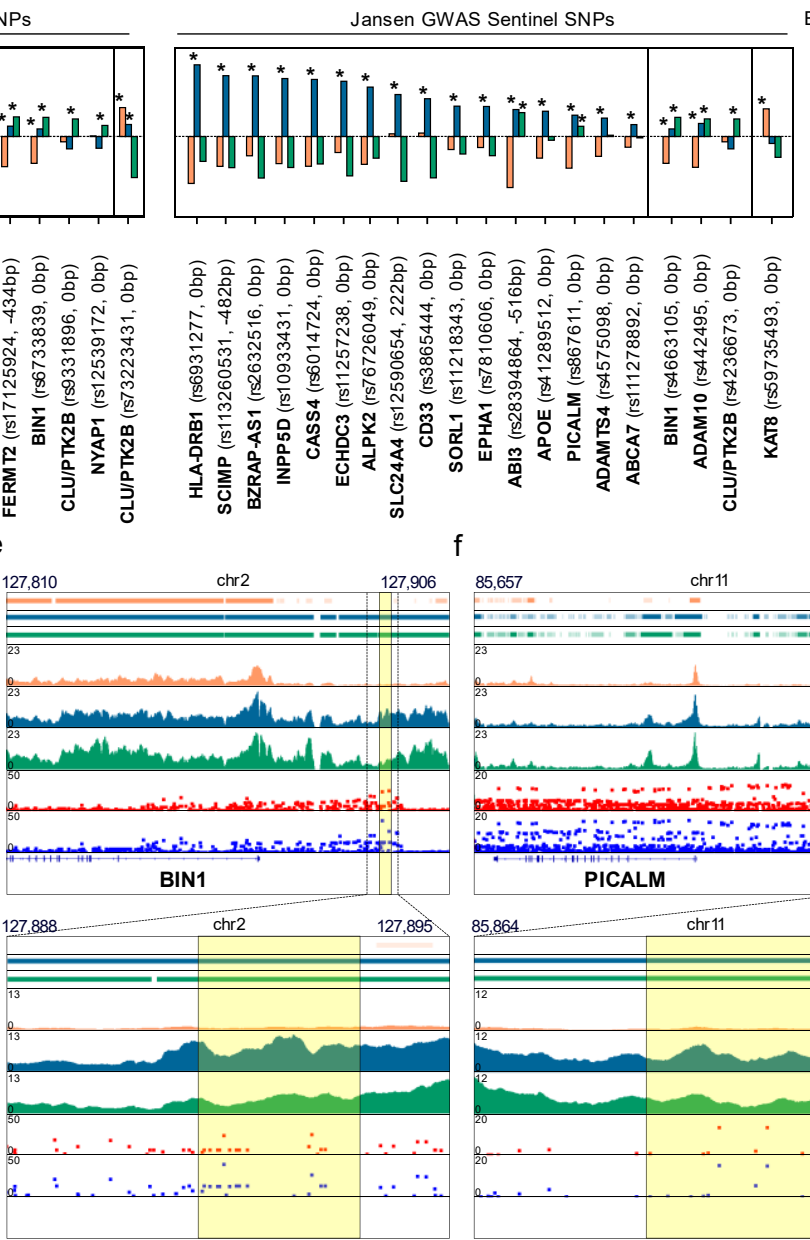

EOAD Genes

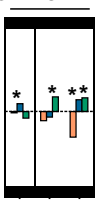

商高高

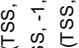

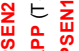

Figure 2: AD associated SNPs derived from GWAS prefer to colocalize with peaks enriched in the microglial population relative to peaks enriched in the OEG and neuronal population a and $b$. Results of stratified LD score regression on two large AD GWAS studies (Jansen et al and Kunkle et al) ${ }^{26,27}$ on hyperacetylated peaks in each population. Plot shows the estimated LD score regression coefficient for the three peak sets. p-values are indicated above each bar. c. Cell type-specificity of peaks annotated to known sentinel non-synonymous SNPs at AD risk loci identified by Jansen and Kunkle et al. Plot shows fold change (log2-transformed) in H3K27ac signal for each population against the other two populations for (i) in black: peaks closest to the sentinel SNP at each locus associated with AD from GWAS, and (ii) in red: promoter peaks of early onset AD risk genes (APP, PSEN1, PSEN2). *indicates DeSeq2 FDR $q<0.05$. Sentinel SNPs where the closest SNP is $>1 \mathrm{~kb}$ away are not included $\mathbf{d , e}$ and $\mathbf{f}$. Genome browser tracks of (i) reproducible peaks in each cell type, (ii) average H3K27ac signal in subjects without $A \beta$ load for each cell type, and (iii) Manhattan plots of Jansen et al and Kunkle et al. GWAS studies at loci where sentinel non-coding SNPs overlap peaks enriched in non-neuronal cell types; at the INPP5D containing locus, the sentinel SNP rs10933431 overlaps a peak that is active only in microglial population but not OEG and neuronal populations; at the locus containing BINI, the top two AD-associated SNPs based on GWAS p-value, rs4663105 and rs6733839 overlap peaks active in the microglial and OEG populations but not in the neuronal population; at the locus containing PICALM, the top two SNPs, rs10792832 and rs3851179 also overlap non-neuronal enhancers. Region of overlap highlighted by yellow box. 
a
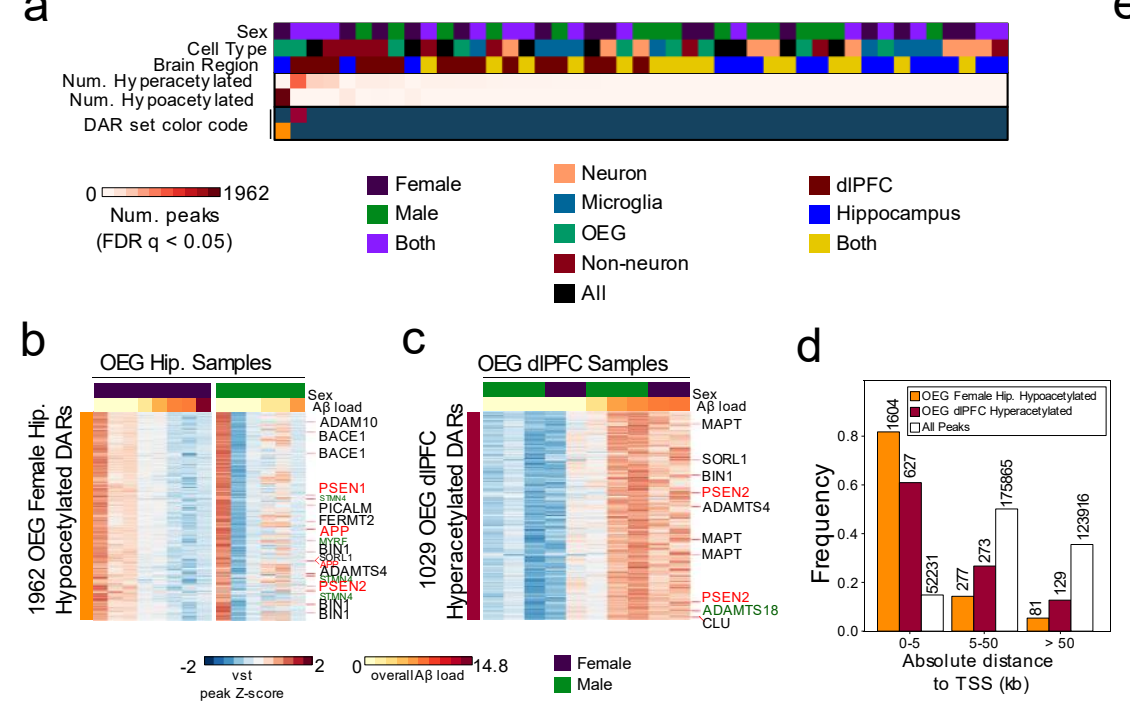

e

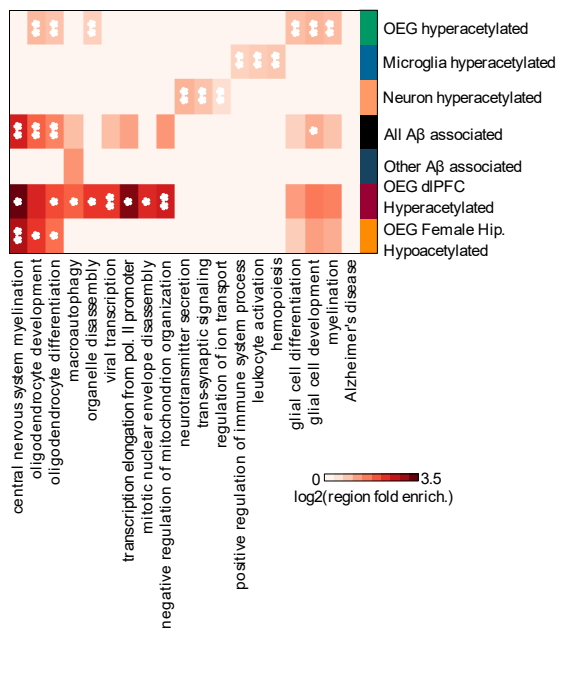

Figure 3: OEG display the strongest acetylation differences associated with A $\beta$ load which includes peaks annotated to known genes associated with EOAD and LOAD risk a. heatmap displaying number of significantly hyperacetylated $(\log 2 \mathrm{fc}>0$, FDR $\mathrm{q}<0.05)$ and significantly hypoacetyled peaks $(\log 2 \mathrm{fc}<0$, FDR $\mathrm{q}<0.05)$ in each stratification of brain region, sex and cell type that was tested $\mathbf{b}$. Heatmap showing normalized acetylation levels at 1962 AD hypoacetylated DARs discovered in female hippocampus OEG samples, rows represent the 1962 DARs and columns represent OEG samples from the hippocampus. Measured A $\beta$ load for each sample is indicated at the bottom of the heatmap, a heatmap for male hippocampal glia samples is also included for comparison, peaks annotated to known EOAD and LOAD risk are labeled in red, peaks near STMN4 (highest hypoacetylation) and MYRF are annotated in black c. heatmap of 1029 DARs displaying hyperacetylation in OEG in dlPFC samples. Peaks annotated to known EOAD and LOAD risk genes are labeled in red, the promoter peak at ADAMTS18 (highest hyperacetylation) is annotated in black d. distance to TSS distribution of i) 1962 OEG female hippocampus hypoacetylated, ii) 1029 OEG dlPFC hyperacetylated DARs and iii) the full set of peaks e. heatmap showing enrichment of top gene ontology terms for 6 peak sets 1) 1962 OEG female hippocampus hypoacetylated 2) 1029 OEG dlPFC hyperacetylated 3) all other A $\beta$ associated DARs 4),5), and 6) neuron, microglia and OEG cell type-specific hyperacetylated peaks. Color intensity represents hypergeometric fold enrichment in number of peaks, * indicates FDR q<0.05, ** indicates FDR q<0.01 


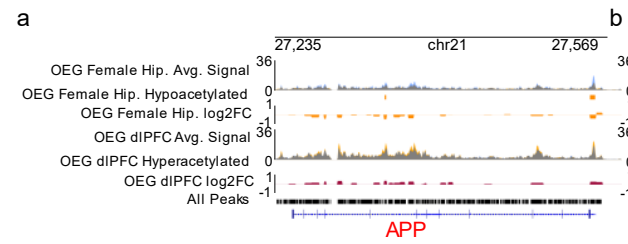

b
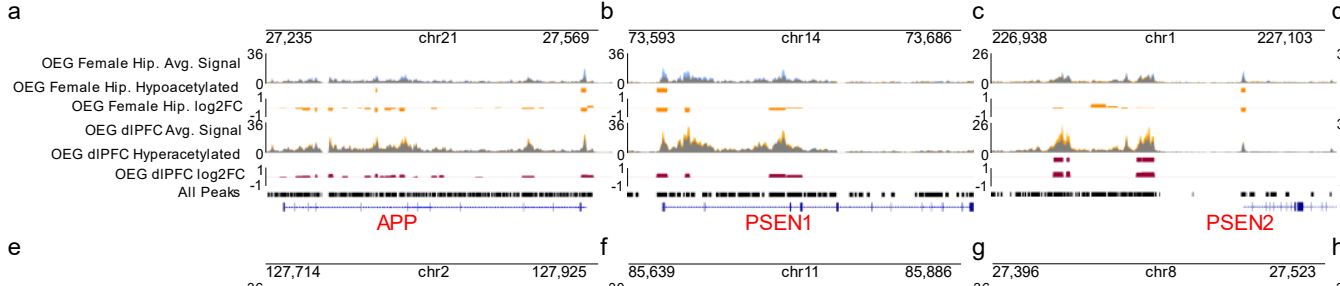

OEG Female Hip. Avg. Signal

OEG Female Hip. $\log 2 \mathrm{FC}$

OEG dIPFC Avg. Signal 36

OEG dIPFC Hyperacetylated 0

OEG dIPFC log2FC
All Peaks

h
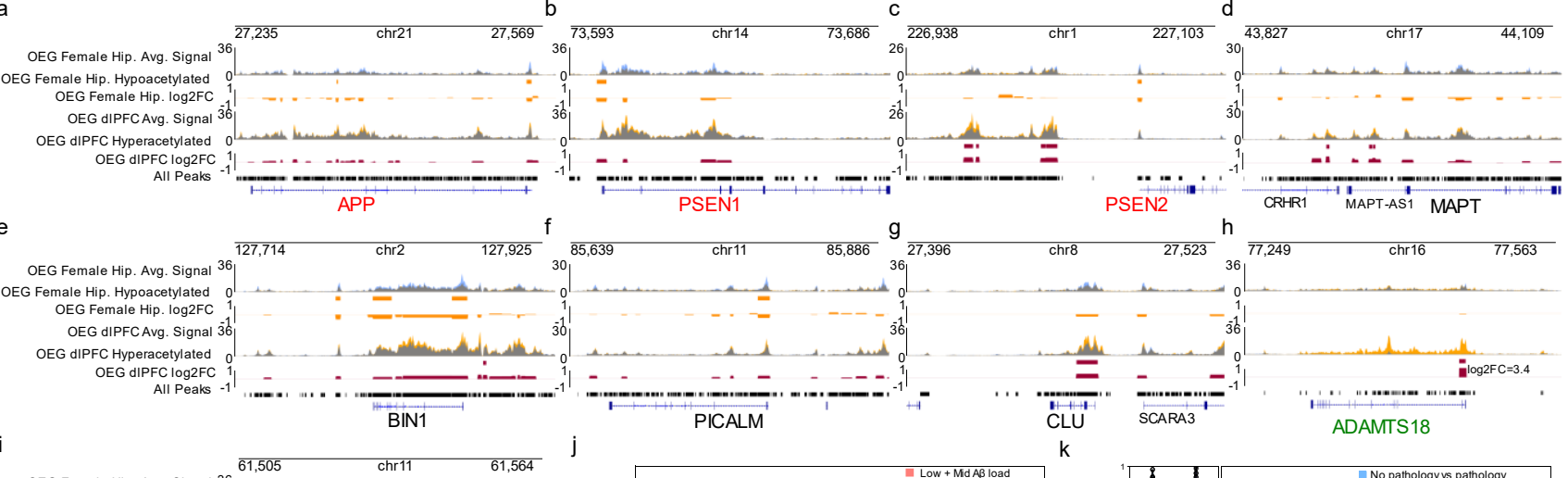

OEG Female Hip. Avg. Signal 36

OEG Female Hip. Hypoacetylated
OEG Female Hip. log2FC

OEG dIPFC Avg. Signal

OEG dIPFC Hyperacetylated

OEG dIPFC log2FC
All Peaks -
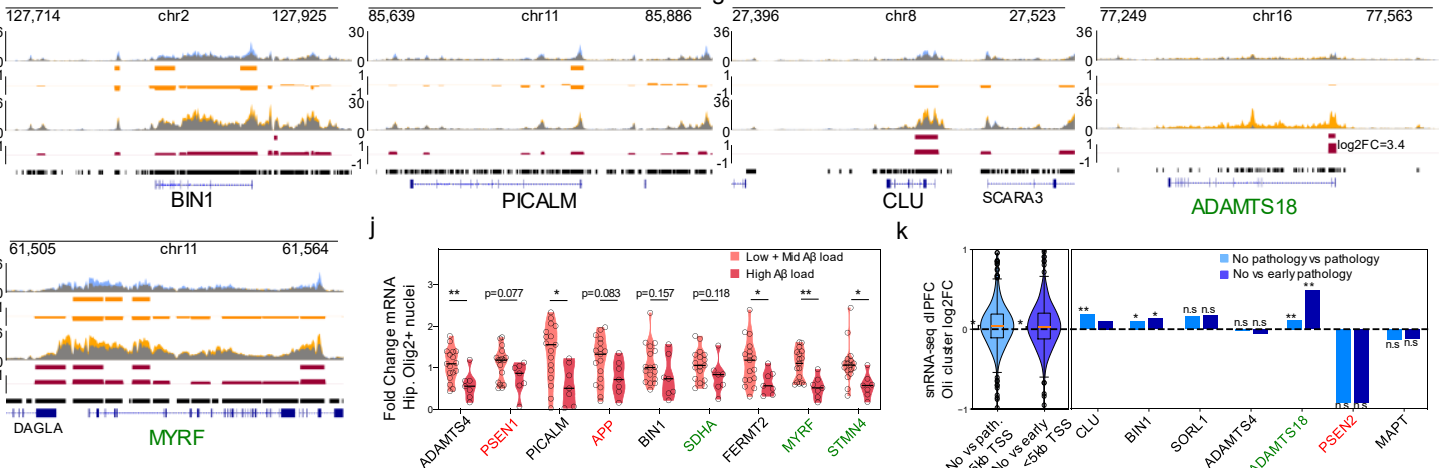

k

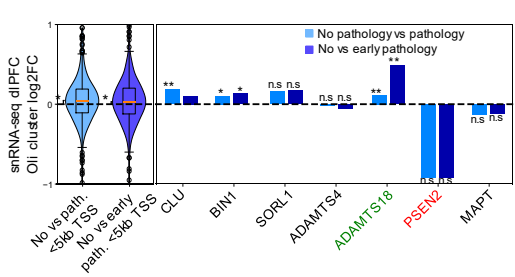

Figure 4: EOAD and LOAD may share common epigenomic perturbations and pathogenic

mechanisms in oligodendrocytes a-i. genome browser tracks displaying average signal in OEG samples of subjects with (yellow) and without $A \beta$ load (blue) corresponding to two biggest DAR sets at known EOAD and LOAD risk loci, as well as strongly differentially acetylated regions near ADAMTS18 and $M Y R F$ j. qRT-PCR in hippocampal Olig2+ nuclei from a larger cohort of subjects reveals an associated decrease in transcription near hypoacetylated regions included in the female hippocampal OEG DAR set k. comparison with existing snRNA-seq from dIPFC of subjects from the same cohort reveals an average increase in gene expression near hyperacetylated regions in OEG dlPFC. Further, specific genes associated with AD risk and displaying high hyperacetylation nearby (ADAMTS18) display an increase in transcription in AD. 
bioRxiv preprint doi: $\mathrm{https} / /$ doi.org/10.1101/2020.03 26.010330; this version posted March 26, 2020. The copyright holder for this preprint (which was not certified by peer review) is the author/funder, who has granted bioRxiv a license to display the preprint in perpetuity. It is made available under aCC-BY 4.0 International license.

\section{SUPPLEMENTARY FIGURES:}
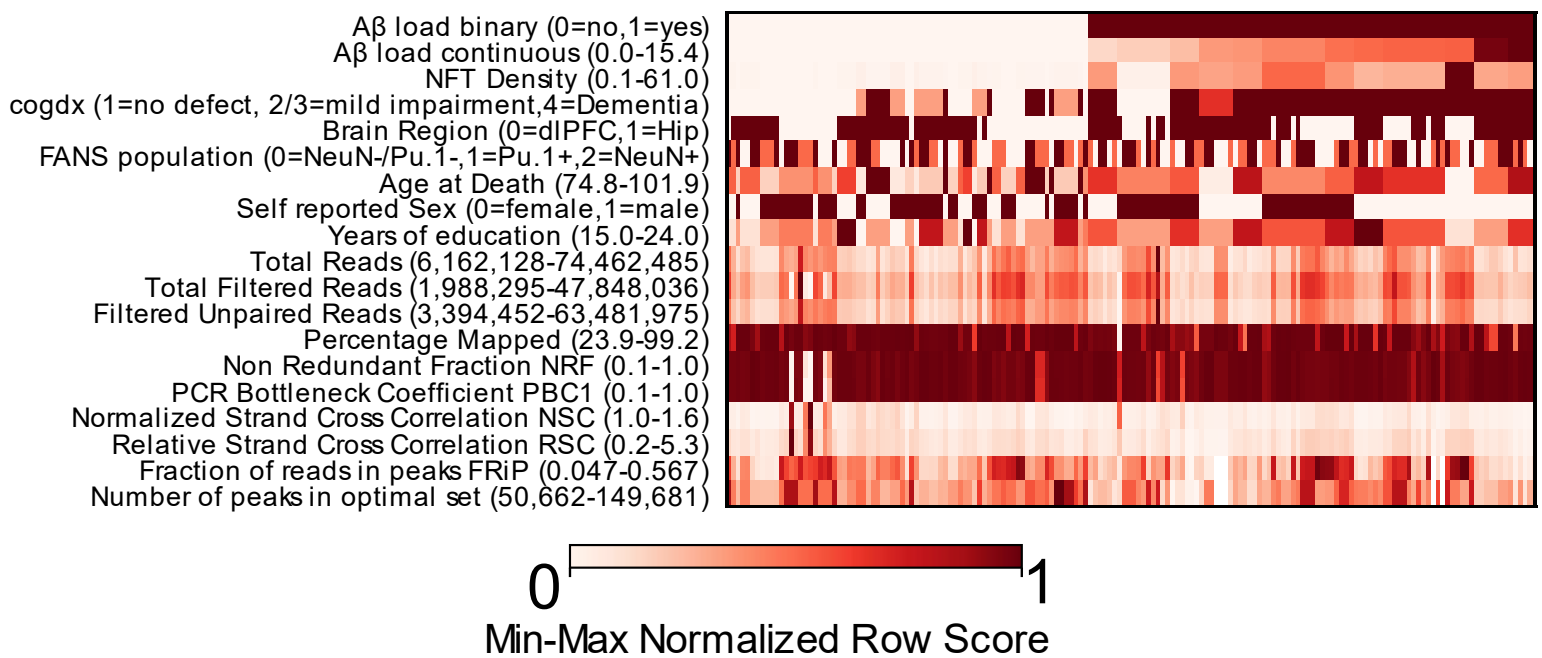

Supplementary Figure 1: Heatmap of collected subject information and standard ENCODE quality metrics for all ChIP-Seq samples
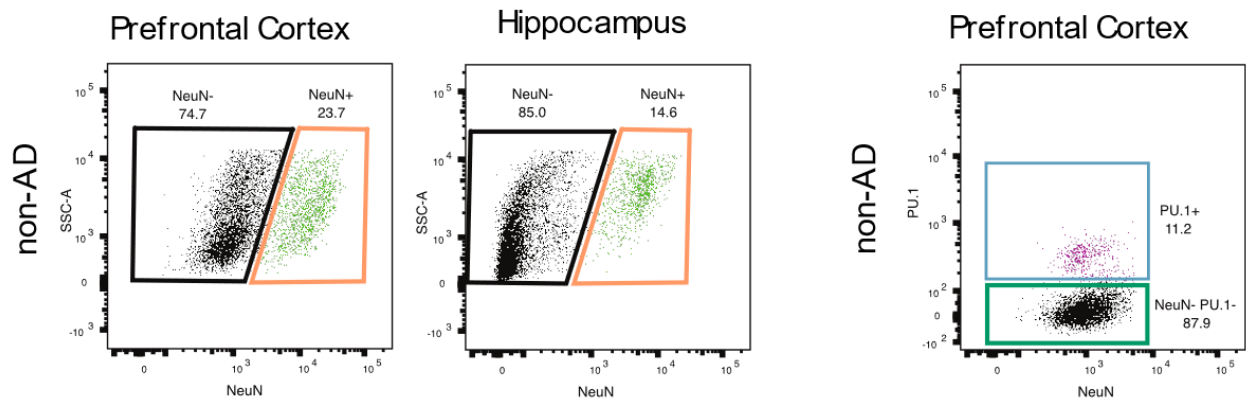

Hippocampus
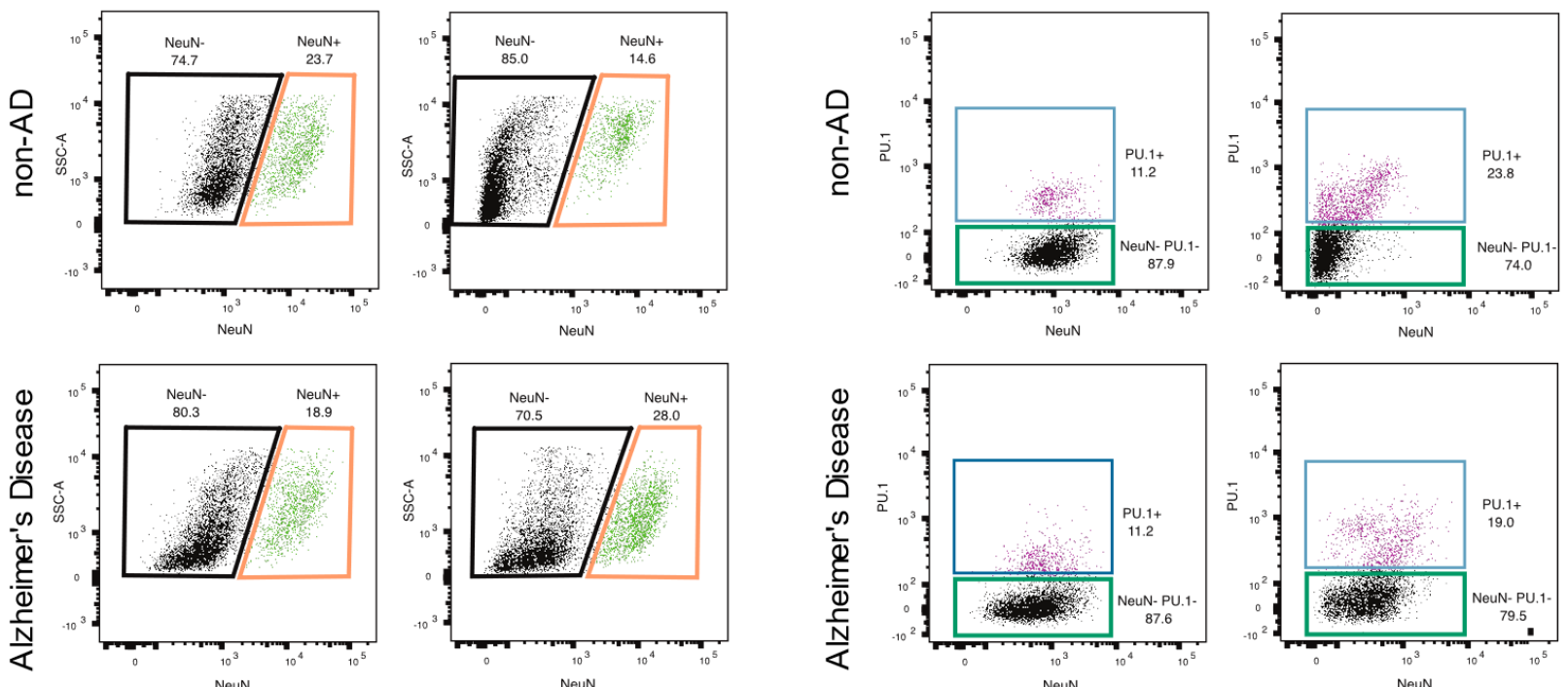

$\mathrm{NeuN}+$ Neurons

PU.1+ Microglia

NeuN- PU.1- Glia

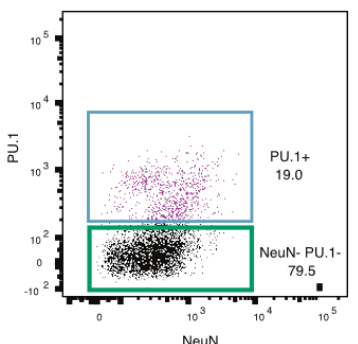

Neun

\section{Supplementary Figure 2: Sorting of neuronal and microglial nuclei from prefrontal cortex and} hippocampus of subjects with and without $\mathrm{AD}$ 

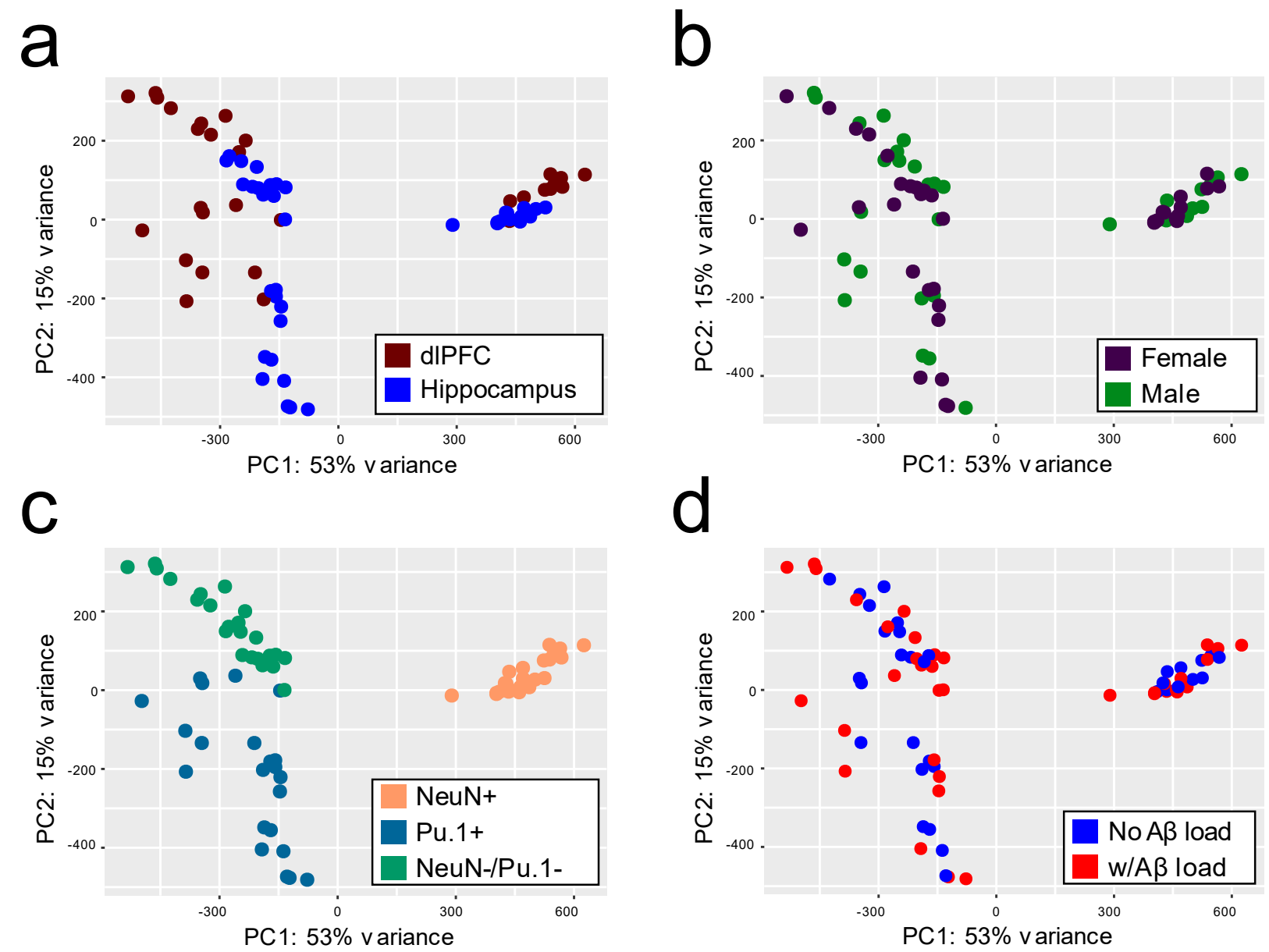

d

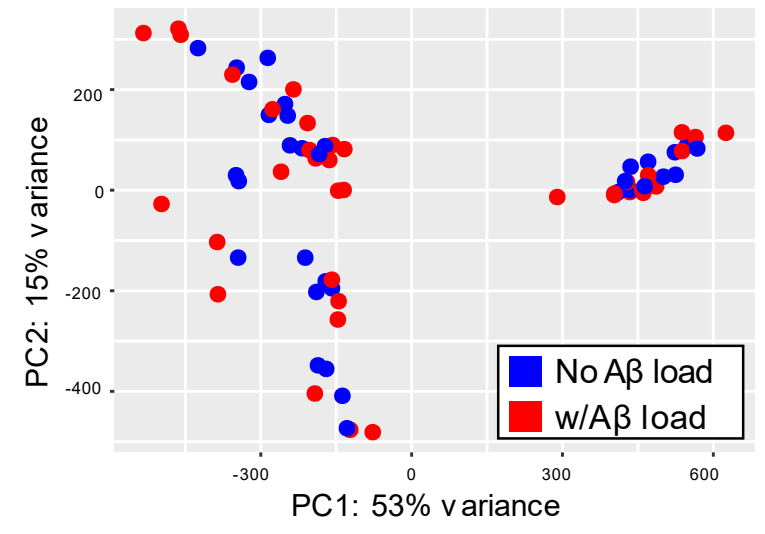

Supplementary Figure 3: Plots showing all sequencing samples projected into principal components space using PCA colored by a. brain region b. sex c. cell type population d. A $\beta$ load PC1 separates $\mathrm{NeuN}+$ samples from Pu.1+ and NeuN-/Pu.1- samples and PC2 separates Pu.1+ samples from NeuN/Pu.1- samples 
a TSS Proximal Peaks $(<5 \mathrm{~kb})$

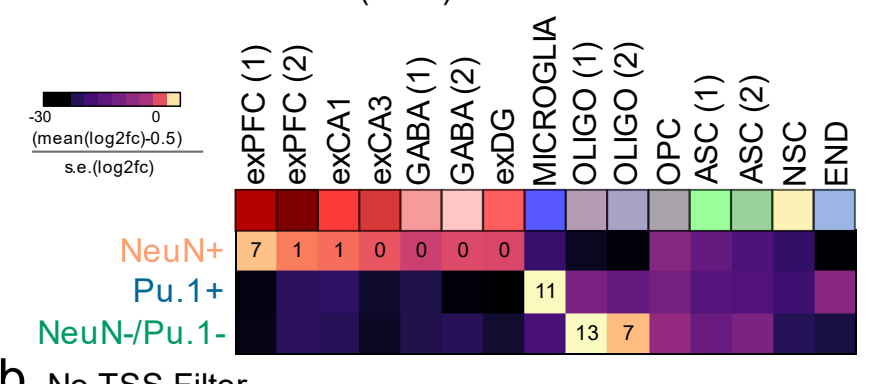

b No TSS Filter

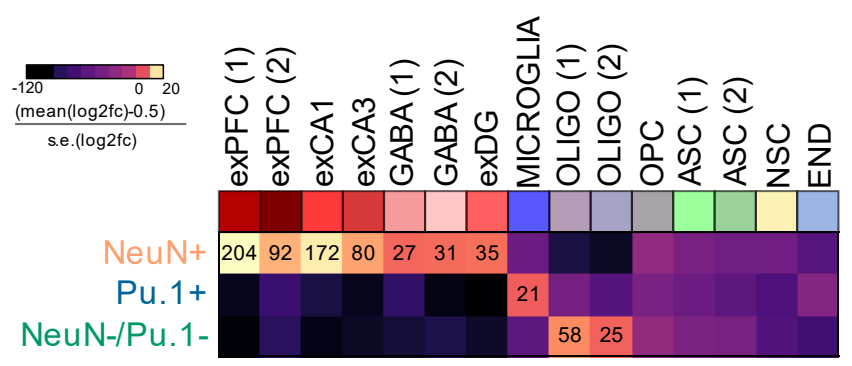

C No TSS Filter

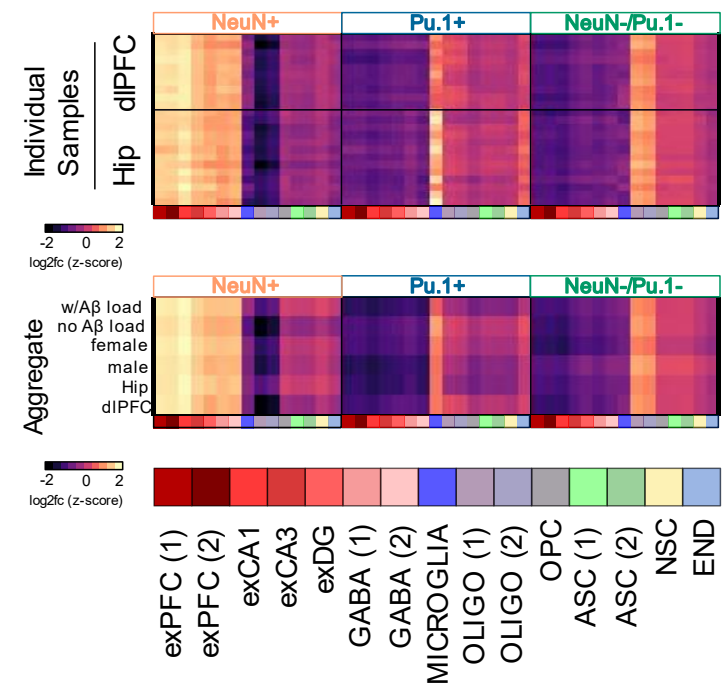

Supplementary Figure 4: Analysis of cell type compositions of FANS populations using H3K27ac signal at peaks near 15 cell type-specific gene lists annotated in Habib et al $^{46}$ a. $t$-statistic showing enrichment of a given cell type cluster in a FANS population over the other two non-focal FANS populations. Mean $\log 2 \mathrm{FC}$ of signal was computed at $\mathrm{H} 3 \mathrm{~K} 27 \mathrm{ac}$ peaks near the promoters $(<5 \mathrm{~kb}$ distance to transcription start site) of the 15 cell type-specific marker gene sets and a t-test was used to compute whether the mean $\log 2 \mathrm{FC}$ is greater than 0.5 ( 1.4-fold change). Value inside box represent p-value ($\log 10$-transformed) for the t-test $\mathbf{b}$. same as panel a but using no distance to tss filter for defining the cell type-specific peaks c. mean $\log 2 \mathrm{fc}$ (focal population/non-focal populations) for peaks near 15 cluster markers at the individual sample level and for sample aggregates using no distance to tss filter for defining the cell type-specific peaks. Abbreviated labels for the single nucleus analysis clusters are presented. exPFC, glutamatergic neurons from the PFC; GABA, GABAergic interneurons; exCA1/3, pyramidal neurons from the hippocampus $\mathrm{CA}$ region; exDG, granule neurons from the hippocampus dentate gyrus region; ASC, astrocytes; MICROGLIA, microglia; OLIGO, oligodendrocytes; OPC, oligodendrocyte precursor cells; NSC, neuronal stem cells; END, endothelial cells. 
a

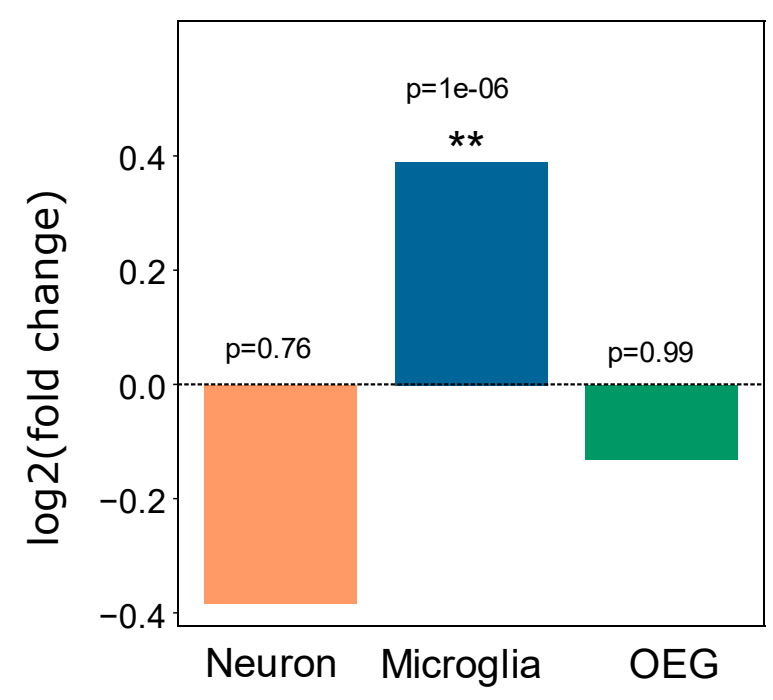

b

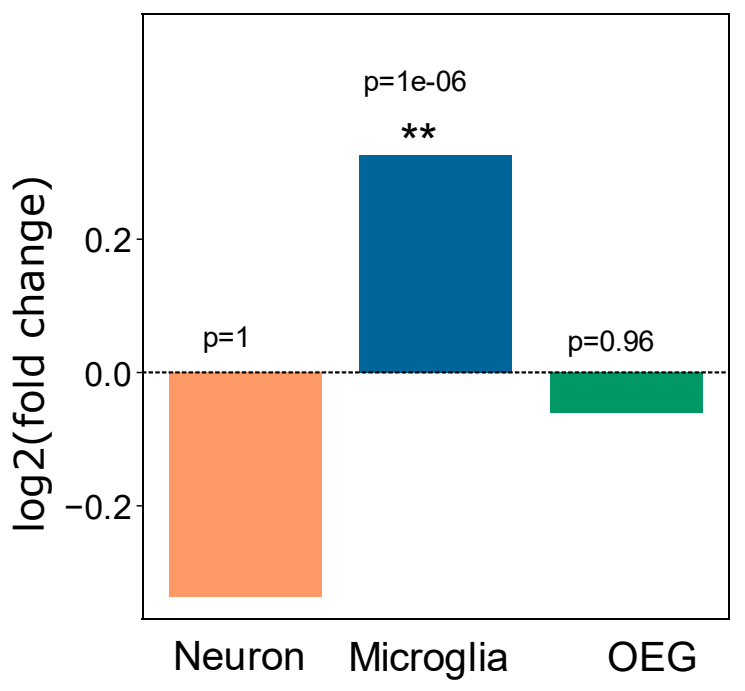

Supplementary Figure 5: Independent permutation test method confirms that AD associated variants from the two large GWAS studies ${ }^{26,27}$ colocalize with peaks enriched in the microglial population relative to peaks enriched in the oligodendrocyte and neuronal population suggesting that microglial gene regulation may influence predisposition towards LOAD a. plot of fold change $(\log 2$ transformed) in the number of AD associated SNPs (Kunkle et al. GWAS p-value < 1e-3) overlapping focal foreground cell type specific peaks versus the number of AD associated SNPs overlapping background set of peaks in all three cell types; permutation test controls for LD and minor allele frequency, p-value for colocalization test is indicated above each bar b. same as a. but using the Jansen et al GWAS. 


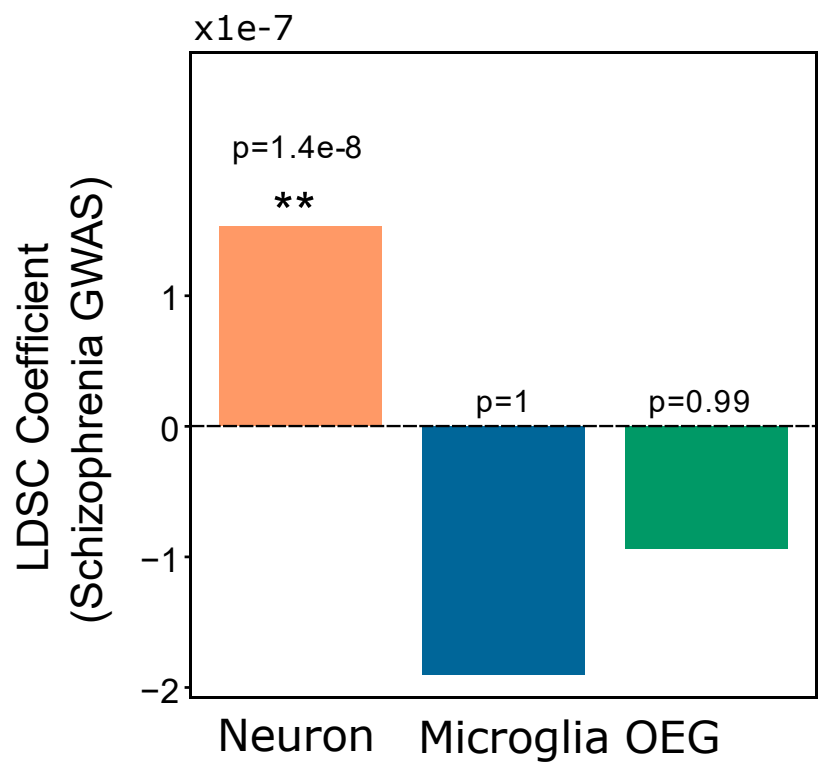

Supplementary Figure 6: SNPs associated with Schizophrenia from a large GWAS $^{53}$ colocalize with peaks enriched in the neuronal population relative to peaks enriched in the OEG and microglial population suggesting that neuronal gene regulation may influence predisposition towards

Schizophrenia Plot shows the estimated stratified LD score regression coefficient for the three peak sets. p-values are indicated above each bar. 
a

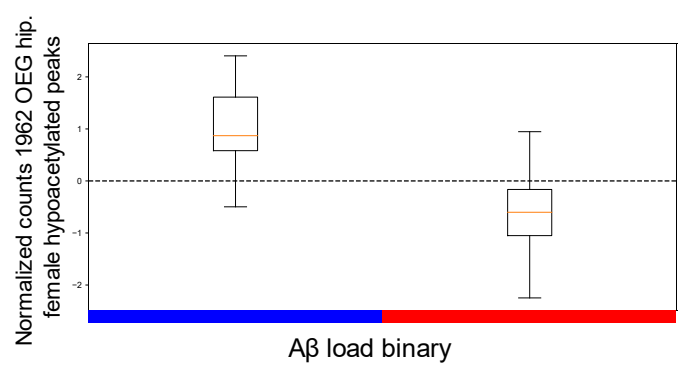

C

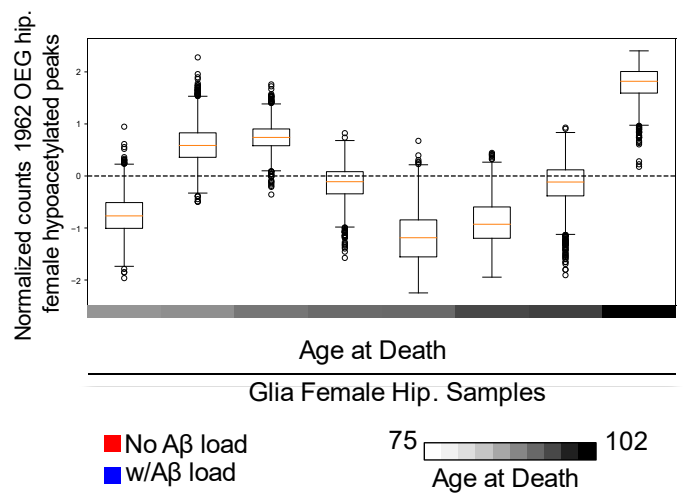

e

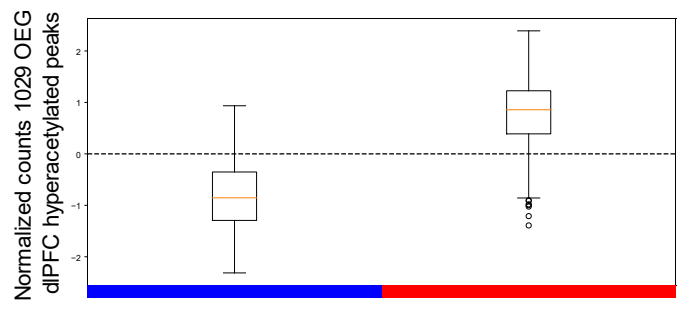

g

$A \beta$ load binary

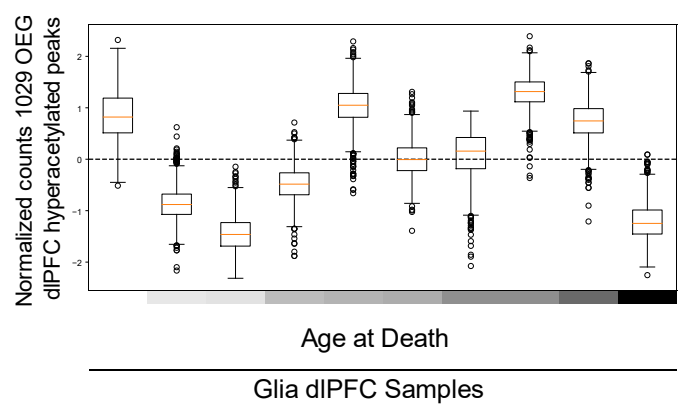

b

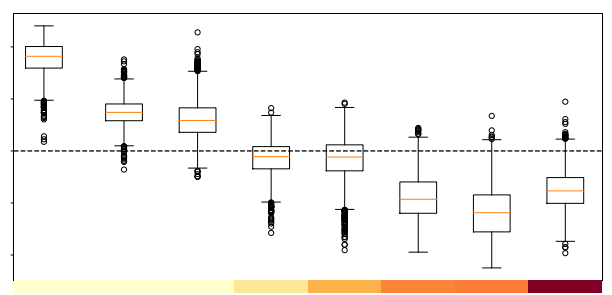

$A \beta$ load continuous

d

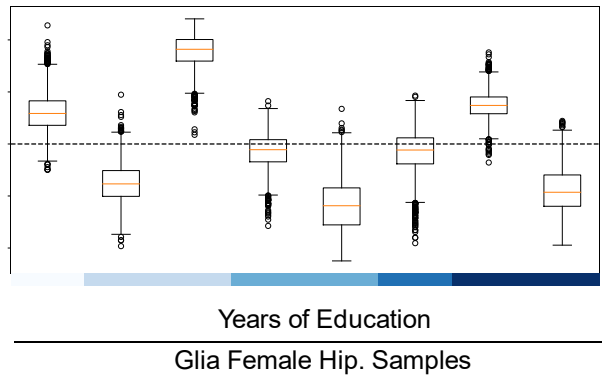

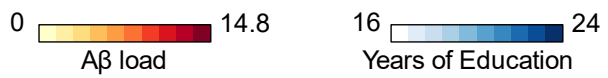

$f$

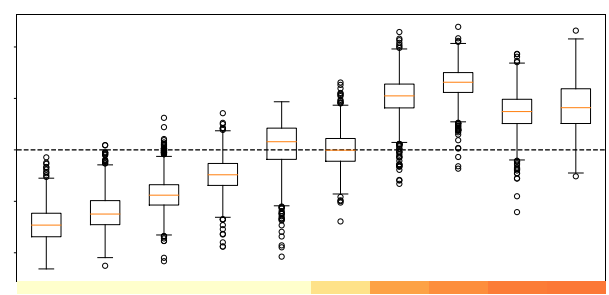

$\mathrm{h}$

$A \beta$ load continuous

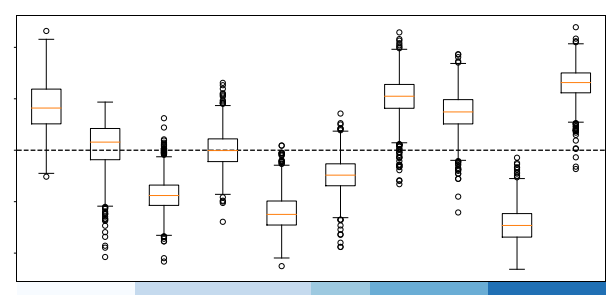

Years of Education

Glia dIPFC Samples

Supplementary Figure 7: Two largest DAR sets of 1962 hypoacetylated peaks in female hippocampus OEG and 1029 hyperacetylated peaks in dIPFC OEG do not show correlations with other covariates such as age at death and years of education. a and b. Read counts at 1962 hypoacetylated DARs show consistent decrease with an increase in overall $A \beta \mathbf{c}$ and d. No relation can be observed between mean read count and variables such as age at death and years of education d,e,f and g. same as a,b,c and d but for 1029 hyperacetylated DARs in dIPFC OEG 


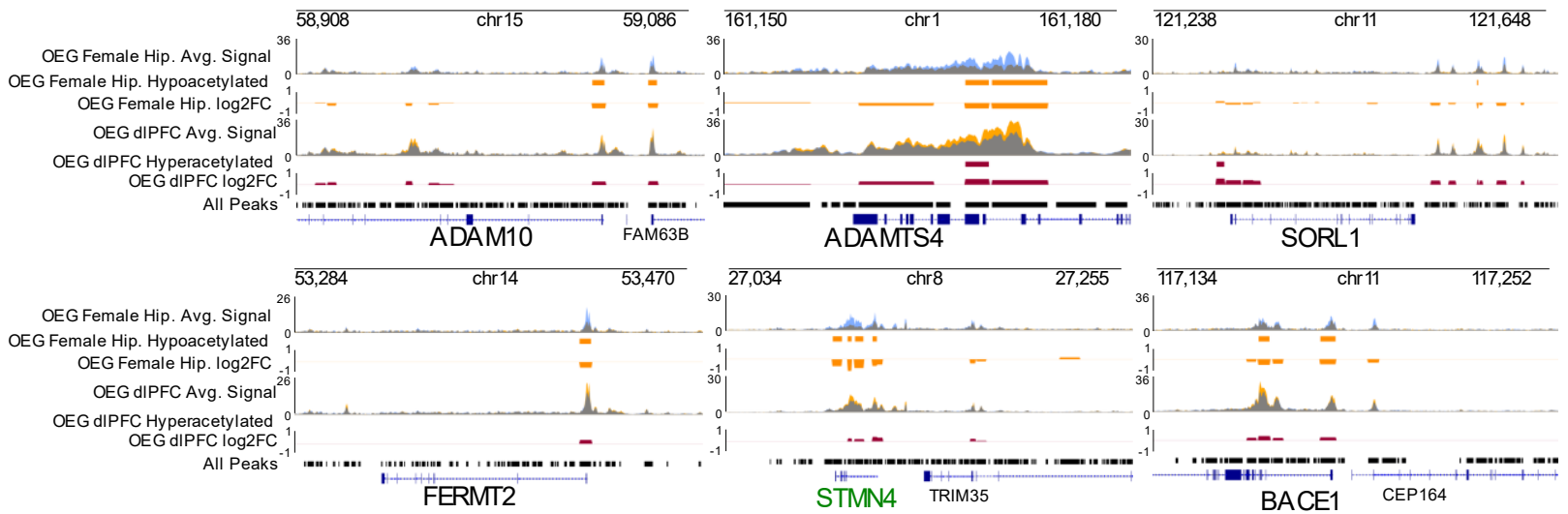

Supplementary Figure 8: Genome browser tracks displaying average H3K27ac signal in OEG samples of subjects with (yellow) and without $A \beta$ load (blue) corresponding to the two biggest DAR sets at known LOAD risk loci, as well as strongly differentially acetylated regions near STMN4 


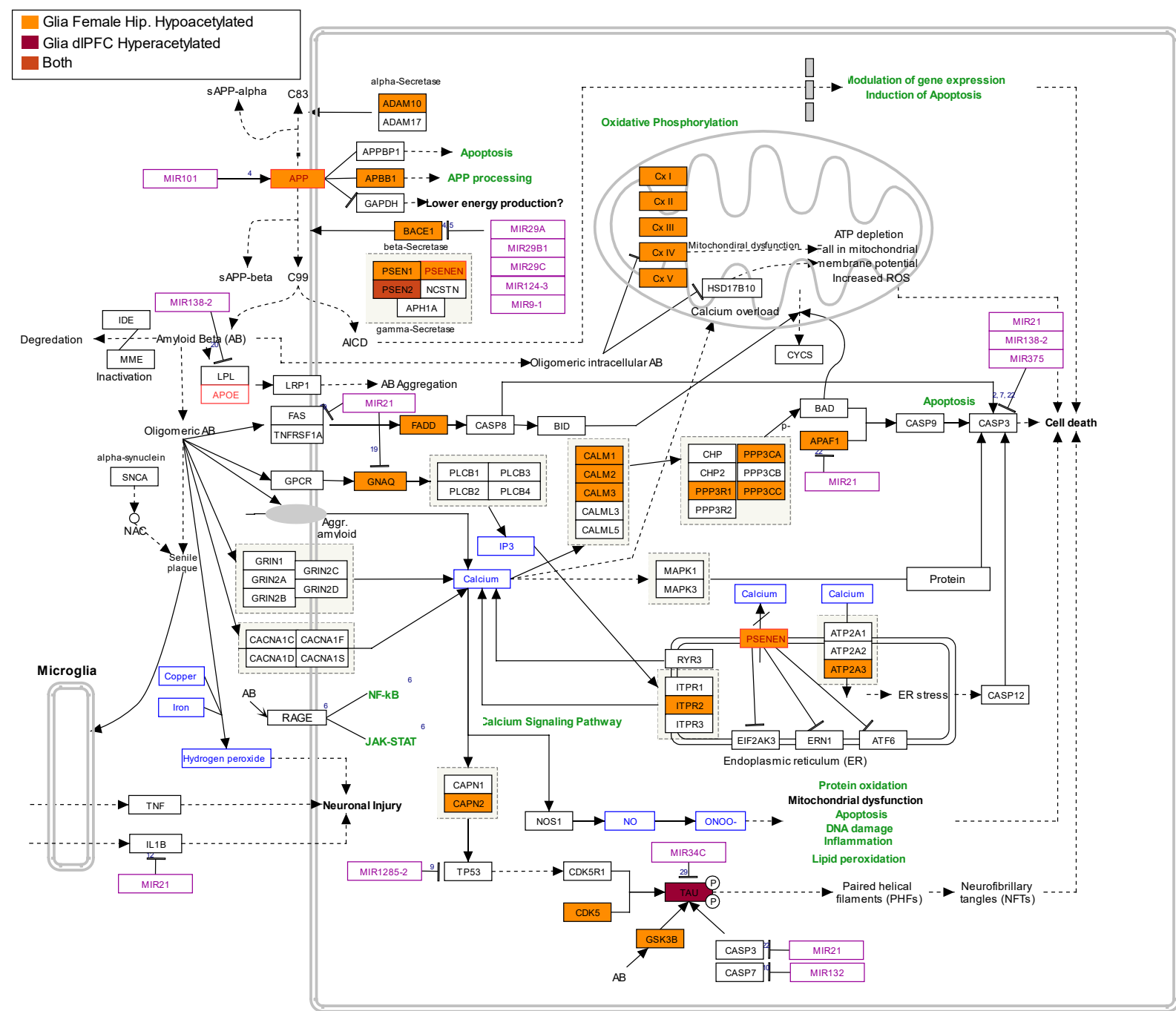

Supplementary Figure 9: Peaks associated with genes in multiple modules in the KEGG Alzheimer's Disease Pathway are part of the two largest OEG specific DAR sets and include peaks near all three of the secretase complexes, MAPT and all 5 modules involved in oxidative phosphorylation Boxes colored in orange represent genes which contain an annotation to the 1962 hypoacetylated peaks discovered in female hippocampus OEG, boxes colored in maroon represent genes which contain an annotation to the 1029 hyperacetylated peaks in dIPFC OEG, an average of orange and maroon is used to color boxes which represent genes present in both sets 

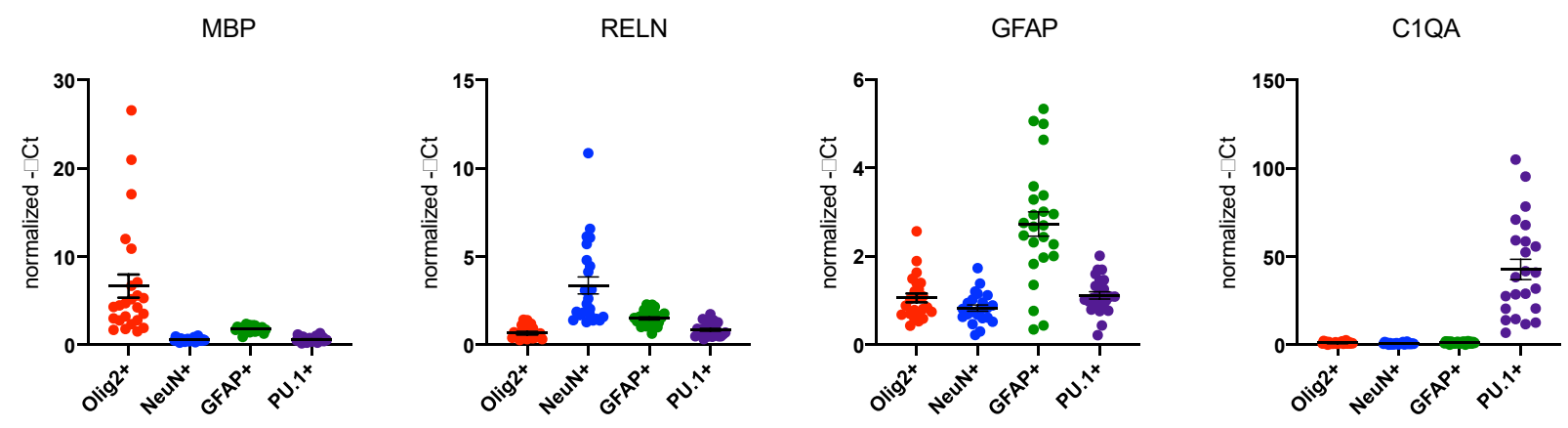

Supplementary Figure 10: mRNA from cell type specific nuclei are enriched for cell type specific markers. Olig2+ nuclei are enriched for oligodendrocyte-specific mRNA MBP, NeuN+ nuclei are enriched for neuron-specific mRNA Reln, GFAP+ nuclei are enriched for astrocyte-specific mRNA GFAP, and Pu.1+ nuclei are enriched for microglia-specific mRNA C1qa. S.E.M. displayed.

a

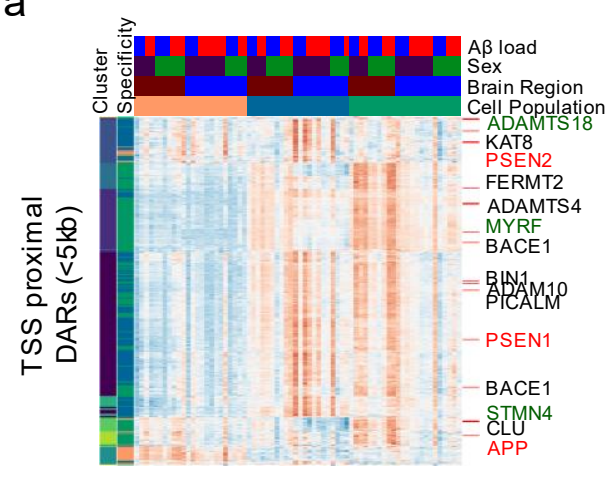

b

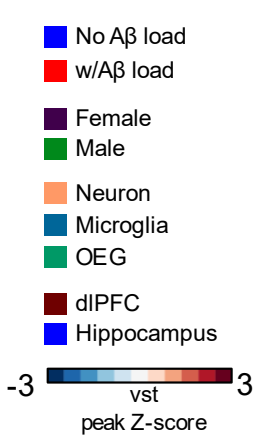

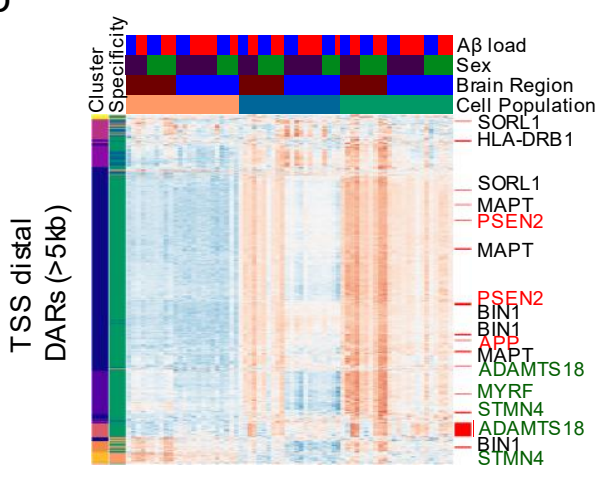

Supplementary Figure 11: a. correlation-based clustering of all TSS proximal DARs identified across different sex, brain region and cell type populations. Heatmap displays normalized acetylation levels at each of the DARs across all samples for every cell type and brain region. Cell type-specificity and cluster membership are indicated for each peak on the left of the heatmap b. same as a but for TSS distal DARs. 
a

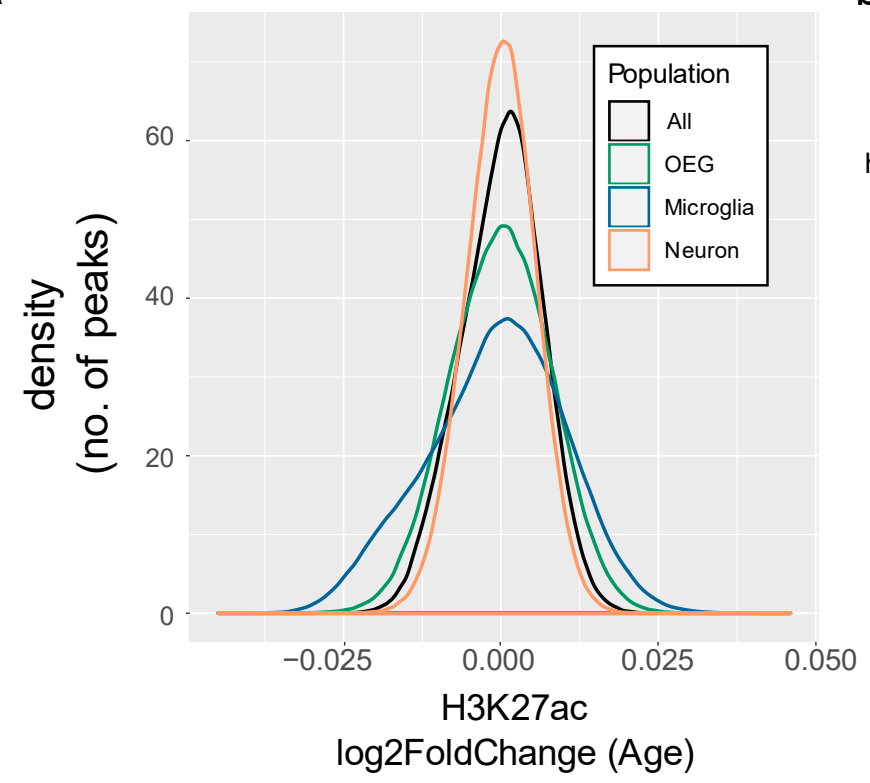

b

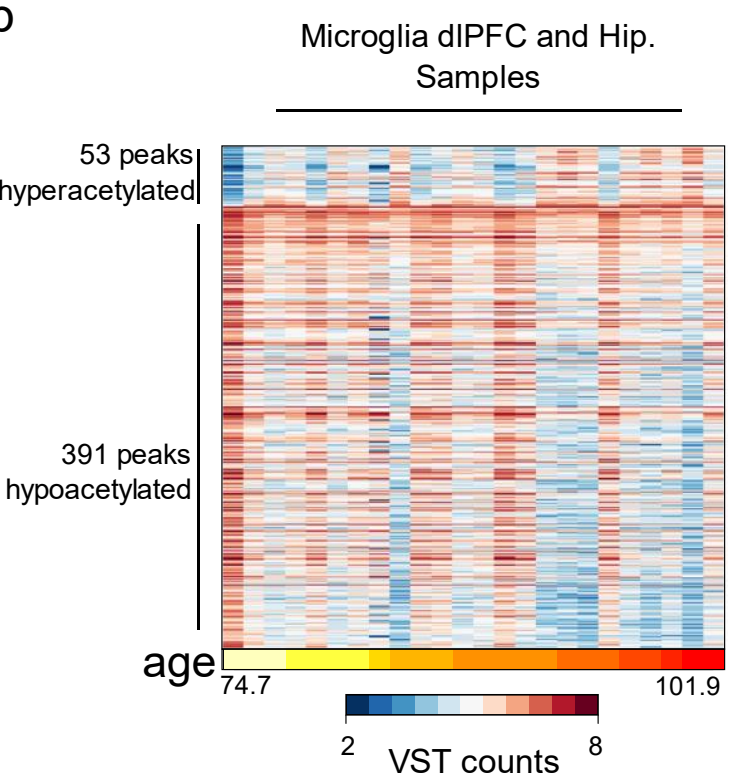

C
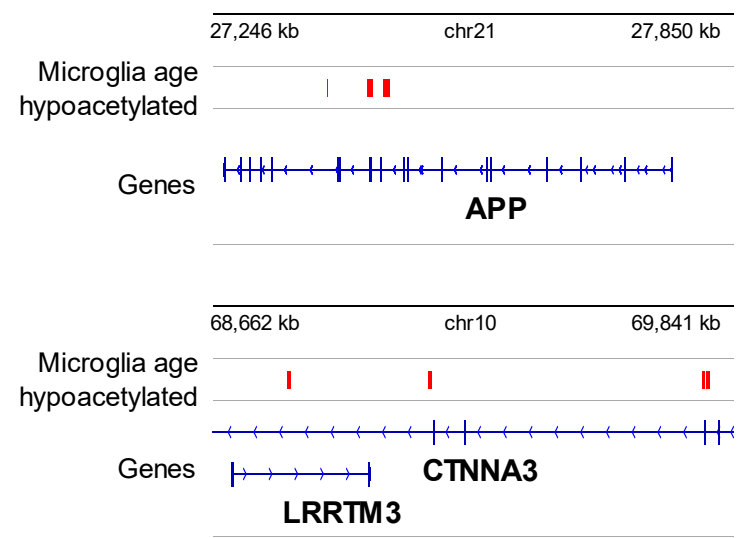

d

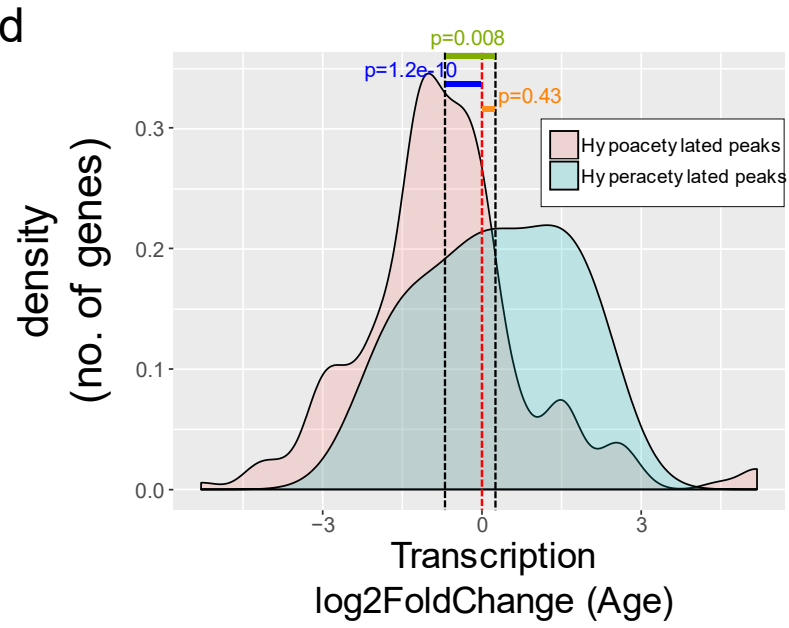

Supplementary Figure 12: Summary of peaks displaying differential H3K27ac with age in microglia a. distribution of age associated $\log 2$ fold changes for the entire peak set shows that age associated changes in $\mathrm{H} 3 \mathrm{~K} 27 \mathrm{ac}$ levels are enriched in the microglial population - as indicated by the wider distribution of $\log 2$ fold changes relative to total, neuronal, and OEG populations, $\mathbf{b}$. heatmap of variance stabilized read counts representing acetylation levels for the 444 peaks that display age associated differential H3K27ac levels in the microglial population, c. Genome browser tracks displaying age hyperacetylated peaks near APP and LRRTM 3 which are both involved in A $\beta$ processing $\mathbf{d}$. distribution of fold change (log2 transformed) in transcription identified in Olah et al ${ }^{97}$ for genes annotated to 391 age hypoacetylated peaks and 53 age hyperacetylated peaks. Two dashed black vertical lines represent mean transcription $\log 2 \mathrm{FC}$ for genes near age hypoacetylated and age hyperacetylated peaks, respectively. Red vertical dashed line represents $\log 2 \mathrm{FC}=0$, representing no difference in transcription. T-test p-values are indicated for three comparisons, from left to right (i) hypoacetylated mean vs. 0, (ii) hyperacetylated vs hypoacetylated means, and (iii) hyperacetylated mean vs. 0 


\section{REFERENCES:}

1. Gaugler, J., James, B., Johnson, T., Scholz, K. \& Weuve, J. 2016 Alzheimer's disease facts and figures. Alzheimer's Dement. 12, 459-509 (2016).

2. Hardy, J. \& Selkoe, D. J. the Amyloid Hypothesis of Alzheimer' S Disease : Progress and Problems on the. Science (80-. ). 297, 353-6 (2002).

3. Goldgaber, D., Lerman, M. I., McBride, W. O., Saffiotti, U. \& Gajdusek, D. C. Isolation, characterization, and chromosomal localization of human brain cDNA clones coding for the precursor of the amyloid of brain in Alzheimer's disease, Down's syndrome and aging. J. Neural Transm. Suppl. 24, 23-8 (1987).

4. Robakis, N. K. et al. Chromosome 21q21 sublocalisation of gene encoding beta-amyloid peptide in cerebral vessels and neuritic (senile) plaques of people with Alzheimer disease and Down syndrome. Lancet (London, England) 1, 384-5 (1987).

5. Kang, J. et al. The precursor of Alzheimer's disease amyloid A4 protein resembles a cell-surface receptor. Nature 325, 733-736 (1987).

6. Tanzi, R. et al. Amyloid beta protein gene: cDNA, mRNA distribution, and genetic linkage near the Alzheimer locus. Science (80-. ). 235, 880-884 (1987).

7. De Strooper, B. Proteases and Proteolysis in Alzheimer Disease: A Multifactorial View on the Disease Process. Physiol. Rev. 90, 465-494 (2010).

8. Levy-Lahad, E. et al. A familial Alzheimer's disease locus on chromosome 1. Science (80-. ). 269, 970-973 (1995).

9. Rogaev, E. I. et al. Familial Alzheimer's disease in kindreds with missense mutations in a gene on chromosome 1 related to the Alzheimer's disease type 3 gene. Nature 376, 775-778 (1995).

10. Schellenberg, G. D. et al. Chromosome 14 and late-onset familial Alzheimer disease (FAD). Am. J. Hum. Genet. 53, 619-28 (1993).

11. Goate, A. Segregation of a missense mutation in the amyloid beta-protein precursor gene with familial Alzheimer's disease. J. Alzheimers. Dis. 9, 341-7 (2006).

12. Goate, A. et al. Segregation of a missense mutation in the amyloid precursor protein gene with familial Alzheimer's disease. Nature 349, 704-706 (1991).

13. St George-Hyslop, P. et al. The genetic defect causing familial Alzheimer's disease maps on chromosome 21. Science (80-. ). 235, 885-890 (1987).

14. Gatz, M. et al. Role of genes and environments for explaining Alzheimer disease. Arch. Gen. Psychiatry 63, 168-174 (2006).

15. Corder, E. et al. Gene dose of apolipoprotein E type 4 allele and the risk of Alzheimer's disease in late onset families. Science (80-. ). 261, 921-923 (1993).

16. Saunders, A. M. et al. Association of apolipoprotein E allele epsilon 4 with late-onset familial and sporadic Alzheimer's disease. Neurology 43, 1467-72 (1993).

17. Saunders, A. M. Apolipoprotein E and Alzheimer Disease: An Update on Genetic and Functional Analyses. J. Neuropathol. Exp. Neurol. 59, 751-758 (2000). 
18. Roses, M.D, A. D. APOLIPOPROTEIN E ALLELES AS RISK FACTORS IN ALZHEIMER'S DISEASE. Annu. Rev. Med. 47, 387-400 (1996).

19. Poirier, J. et al. Apolipoprotein E polymorphism and Alzheimer's disease. Lancet (London, England) 342, 697-9 (1993).

20. Naj, A. C. et al. Common variants at MS4A4/MS4A6E, CD2AP, CD33 and EPHA1 are associated with late-onset Alzheimer's disease. Nat. Genet. 43, 436-441 (2011).

21. Hollingworth, P. et al. Common variants at ABCA7, MS4A6A/MS4A4E, EPHA1, CD33 and CD2AP are associated with Alzheimer's disease. Nat. Genet. 43, 429-435 (2011).

22. Seshadri, S. et al. Genome-wide Analysis of Genetic Loci Associated With Alzheimer Disease. JAMA 303, 1832 (2010).

23. Lambert, J.-C. et al. Genome-wide association study identifies variants at CLU and CR1 associated with Alzheimer's disease. Nat. Genet. 41, 1094-1099 (2009).

24. Harold, D. et al. Genome-wide association study identifies variants at CLU and PICALM associated with Alzheimer's disease. Nat. Genet. 41, 1088-1093 (2009).

25. Lambert, J.-C. et al. Meta-analysis of 74,046 individuals identifies 11 new susceptibility loci for Alzheimer's disease. Nat. Genet. 45, 1452-1458 (2013).

26. Kunkle, B. W. et al. Genetic meta-analysis of diagnosed Alzheimer's disease identifies new risk loci and implicates A $\beta$, tau, immunity and lipid processing. Nat. Genet. 51, 414-430 (2019).

27. Jansen, I. E. et al. Genome-wide meta-analysis identifies new loci and functional pathways influencing Alzheimer's disease risk. Nat. Genet. 1 (2019). doi:10.1038/s41588-018-0311-9

28. Bertram, L. \& Tanzi, R. E. Alzheimer disease risk genes: 29 and counting. Nat. Rev. Neurol. 15, 191-192 (2019).

29. Ward, L. D. \& Kellis, M. HaploReg: a resource for exploring chromatin states, conservation, and regulatory motif alterations within sets of genetically linked variants. Nucleic Acids Res. 40, D930-D934 (2012).

30. Gjoneska, E. et al. Conserved epigenomic signals in mice and humans reveal immune basis of Alzheimer's disease. Nature 518, 365-369 (2015).

31. Nott, A. et al. Brain cell type-specific enhancer-promoter interactome maps and disease-risk association. Science (80-. ). 366, 1134-1139 (2019).

32. Marzi, S. J. et al. A histone acetylome-wide association study of Alzheimer's disease identifies disease-associated H3K27ac differences in the entorhinal cortex. Nat. Neurosci. 21, 1618-1627 (2018).

33. Nativio, R. et al. Dysregulation of the epigenetic landscape of normal aging in Alzheimer's disease. Nat. Neurosci. 21, 497-505 (2018).

34. Klein, H.-U. et al. Epigenome-wide study uncovers large-scale changes in histone acetylation driven by tau pathology in aging and Alzheimer's human brains. Nat. Neurosci. 22, 37-46 (2019).

35. Kundaje, A. et al. Integrative analysis of 111 reference human epigenomes. Nature 518, 317-330 (2015).

36. Huang, K. et al. A common haplotype lowers PU.1 expression in myeloid cells and delays onset of 
Alzheimer's disease. Nat. Neurosci. 20, 1052-1061 (2017).

37. Gosselin, D. et al. An environment-dependent transcriptional network specifies human microglia identity. Science (80-. ). 356, eaal3222 (2017).

38. Lake, B. B. et al. Integrative single-cell analysis of transcriptional and epigenetic states in the human adult brain. Nat. Biotechnol. 36, 70-80 (2017).

39. Marion-Poll, L., Montalban, E., Munier, A., Hervé, D. \& Girault, J.-A. Fluorescence-activated sorting of fixed nuclei: a general method for studying nuclei from specific cell populations that preserves post-translational modifications. Eur. J. Neurosci. 39, 1234-1244 (2014).

40. Creyghton, M. P. et al. Histone H3K27ac separates active from poised enhancers and predicts developmental state. Proc. Natl. Acad. Sci. U. S. A. 107, 21931-6 (2010).

41. Bennett, D. A. et al. Selected Findings from the Religious Orders Study and Rush Memory and Aging Project. J. Alzheimer's Dis. 33, S397-S403 (2012).

42. Bennett, D. A. et al. Overview and findings from the rush Memory and Aging Project. Curr. Alzheimer Res. 9, 646-63 (2012).

43. Bennett, D. A. et al. Religious Orders Study and Rush Memory and Aging Project. Journal of Alzheimer's Disease 64, S161-S189 (2018).

44. Landt, S. G. et al. ChIP-seq guidelines and practices of the ENCODE and modENCODE consortia. Genome Res. 22, 1813-1831 (2012).

45. Love, M. I., Huber, W. \& Anders, S. Moderated estimation of fold change and dispersion for RNA-seq data with DESeq2. Genome Biol. 15, 550 (2014).

46. Habib, N. et al. Massively parallel single-nucleus RNA-seq with DroNc-seq. Nat. Methods 14, 955-958 (2017).

47. Bulik-Sullivan, B. K. et al. LD Score regression distinguishes confounding from polygenicity in genome-wide association studies. Nat. Genet. 47, 291-295 (2015).

48. Finucane, H. K. et al. Partitioning heritability by functional annotation using genome-wide association summary statistics. Nat. Genet. 47, 1228-1235 (2015).

49. Finucane, H. K. et al. Heritability enrichment of specifically expressed genes identifies diseaserelevant tissues and cell types. Nat. Genet. 50, 621-629 (2018).

50. Gjoneska, E. et al. Conserved epigenomic signals in mice and humans reveal immune basis of Alzheimer's disease. Nature 518, 365-369 (2015).

51. Huang, K. et al. A common haplotype lowers PU.1 expression in myeloid cells and delays onset of Alzheimer's disease. Nat. Neurosci. 20, 1052-1061 (2017).

52. Keren-Shaul, H. et al. A Unique Microglia Type Associated with Restricting Development of Alzheimer's Disease. Cell 169, 1276-1290.e17 (2017).

53. Biological insights from 108 schizophrenia-associated genetic loci. Nature 511, 421-427 (2014).

54. Fullard, J. F. et al. Open chromatin profiling of human postmortem brain infers functional roles for non-coding schizophrenia loci. Hum. Mol. Genet. 26, 1942-1951 (2017).

55. Fullard, J. F. et al. An atlas of chromatin accessibility in the adult human brain. Genome Res. 28, 1243-1252 (2018). 
56. Kheradpour, P. \& Kellis, M. Systematic discovery and characterization of regulatory motifs in ENCODE TF binding experiments. Nucleic Acids Res. 42, 2976-2987 (2014).

57. Ardlie, K. G. et al. The Genotype-Tissue Expression (GTEx) pilot analysis: Multitissue gene regulation in humans. Science (80-. ). 348, 648-660 (2015).

58. Lappalainen, T. et al. Transcriptome and genome sequencing uncovers functional variation in humans. Nature 501, 506-511 (2013).

59. McLean, C. Y. et al. GREAT improves functional interpretation of cis-regulatory regions. Nat. Biotechnol. 28, 495-501 (2010).

60. Fagerberg, L. et al. Analysis of the Human Tissue-specific Expression by Genome-wide Integration of Transcriptomics and Antibody-based Proteomics. Mol. Cell. Proteomics 13, 397406 (2014).

61. Gaudet, P., Livstone, M. S., Lewis, S. E. \& Thomas, P. D. Phylogenetic-based propagation of functional annotations within the Gene Ontology consortium. Brief. Bioinform. 12, 449-62 (2011).

62. Bujalka, H. et al. MYRF Is a Membrane-Associated Transcription Factor That Autoproteolytically Cleaves to Directly Activate Myelin Genes. PLoS Biol. 11, e1001625 (2013).

63. Vardarajan, B. N. et al. Whole genome sequencing of Caribbean Hispanic families with late-onset Alzheimer's disease. Ann. Clin. Transl. Neurol. 5, 406-417 (2018).

64. Mathys, H. et al. Single-cell transcriptomic analysis of Alzheimer's disease. Nature 570, 332-337 (2019).

65. Gurses, M. S., Ural, M. N., Gulec, M. A., Akyol, O. \& Akyol, S. Pathophysiological Function of ADAMTS Enzymes on Molecular Mechanism of Alzheimer's Disease. Aging Dis. 7, 479 (2016).

66. Ran, F. A. et al. Genome engineering using the CRISPR-Cas9 system. Nat. Protoc. 8, 2281-2308 (2013).

67. Mathys, H. et al. Temporal Tracking of Microglia Activation in Neurodegeneration at Single-Cell Resolution. Cell Rep. 21, 366-380 (2017).

68. Alsema, A. M. et al. Profiling microglia from AD donors and non-demented elderly in acute human post-mortem cortical tissue. bioRxiv 2020.03.18.995332 (2020). doi:10.1101/2020.03.18.995332

69. Seyfried, N. T. et al. A Multi-network Approach Identifies Protein-Specific Co-expression in Asymptomatic and Symptomatic Alzheimer's Disease. Cell Syst. 4, 60-72.e4 (2017).

70. McKenzie, A. T. et al. Multiscale network modeling of oligodendrocytes reveals molecular components of myelin dysregulation in Alzheimer's disease. Mol. Neurodegener. 12, 82 (2017).

71. De Rossi, P. et al. Predominant expression of Alzheimer's disease-associated BIN1 in mature oligodendrocytes and localization to white matter tracts. Mol. Neurodegener. 11, (2016).

72. Bartzokis, G. Alzheimer's disease as homeostatic responses to age-related myelin breakdown. Neurobiology of Aging 32, 1341-1371 (2011).

73. Marioni, R. E. et al. GWAS on family history of Alzheimer's disease. Transl. Psychiatry 8, 99 (2018).

74. Braak, H., Braak, E. \& Bohl, J. Staging of Alzheimer-Related Cortical Destruction. Eur. Neurol. 
33, 403-408 (1993).

75. de Leeuw, F.-E., Barkhof, F. \& Scheltens, P. White matter lesions and hippocampal atrophy in Alzheimer's disease. Neurology 62, 310-2 (2004).

76. Lee, S. et al. White matter hyperintensities are a core feature of Alzheimer's disease: Evidence from the dominantly inherited Alzheimer network. Ann. Neurol. 79, 929-939 (2016).

77. Grady, C. L. et al. Evidence from functional neuroimaging of a compensatory prefrontal network in Alzheimer's disease. J. Neurosci. 23, 986-993 (2003).

78. Barres, B. A. The Mystery and Magic of Glia: A Perspective on Their Roles in Health and Disease. Neuron 60, 430-440 (2008).

79. Nave, K.-A. \& Trapp, B. D. Axon-Glial Signaling and the Glial Support of Axon Function. Annu. Rev. Neurosci. 31, 535-561 (2008).

80. Freeman, M. R. \& Doherty, J. Glial cell biology in Drosophila and vertebrates. Trends Neurosci. 29, 82-90 (2006).

81. Allen, N. J. \& Barres, B. A. Signaling between glia and neurons: focus on synaptic plasticity. Curr. Opin. Neurobiol. 15, 542-548 (2005).

82. Li, H. \& Durbin, R. Fast and accurate short read alignment with Burrows-Wheeler transform. Bioinformatics 25, 1754-1760 (2009).

83. Li, H. et al. The Sequence Alignment/Map format and SAMtools. Bioinformatics 25, 2078-2079 (2009).

84. Zhang, Y. et al. Model-based Analysis of ChIP-Seq (MACS). Genome Biol. 9, R137 (2008).

85. Li, Q., Brown, J. B., Huang, H. \& Bickel, P. J. Measuring reproducibility of high-throughput experiments. Ann. Appl. Stat. 5, 1752-1779 (2011).

86. Quinlan, A. R. \& Hall, I. M. BEDTools: a flexible suite of utilities for comparing genomic features. Bioinformatics 26, 841-842 (2010).

87. Ernst, J. et al. Mapping and analysis of chromatin state dynamics in nine human cell types. Nature 473, 43-49 (2011).

88. Liao, Y., Smyth, G. K. \& Shi, W. featureCounts: an efficient general purpose program for assigning sequence reads to genomic features. Bioinformatics 30, 923-930 (2014).

89. Robinson, J. T. et al. Integrative genomics viewer. Nat. Biotechnol. 29, 24-26 (2011).

90. Ramírez, F. et al. High-resolution TADs reveal DNA sequences underlying genome organization in flies. Nat. Commun. 9, 189 (2018).

91. Heinz, S. et al. Simple Combinations of Lineage-Determining Transcription Factors Prime cisRegulatory Elements Required for Macrophage and B Cell Identities. Mol. Cell 38, 576-589 (2010).

92. Gibbs, R. A. et al. A global reference for human genetic variation. Nature 526, 68-74 (2015).

93. Zerbino, D. R. et al. Ensembl 2018. Nucleic Acids Res. 46, D754-D761 (2018).

94. Kent, W. J., Zweig, A. S., Barber, G., Hinrichs, A. S. \& Karolchik, D. BigWig and BigBed: enabling browsing of large distributed datasets. Bioinformatics 26, 2204-2207 (2010). 
95. Karolchik, D. et al. The UCSC Table Browser data retrieval tool. Nucleic Acids Res. 32, 493D 496 (2004).

96. Langfelder, P., Zhang, B. \& Horvath, S. Defining clusters from a hierarchical cluster tree: the Dynamic Tree Cut package for R. Bioinformatics 24, 719-720 (2008).

97. Olah, M. et al. A transcriptomic atlas of aged human microglia. Nat. Commun. 9, 539 (2018). 
Extraction of Brain Tissue: Hippocampus

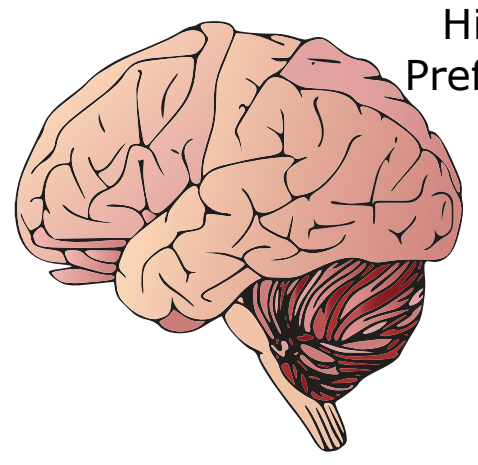
Prefrontal Cortex

b

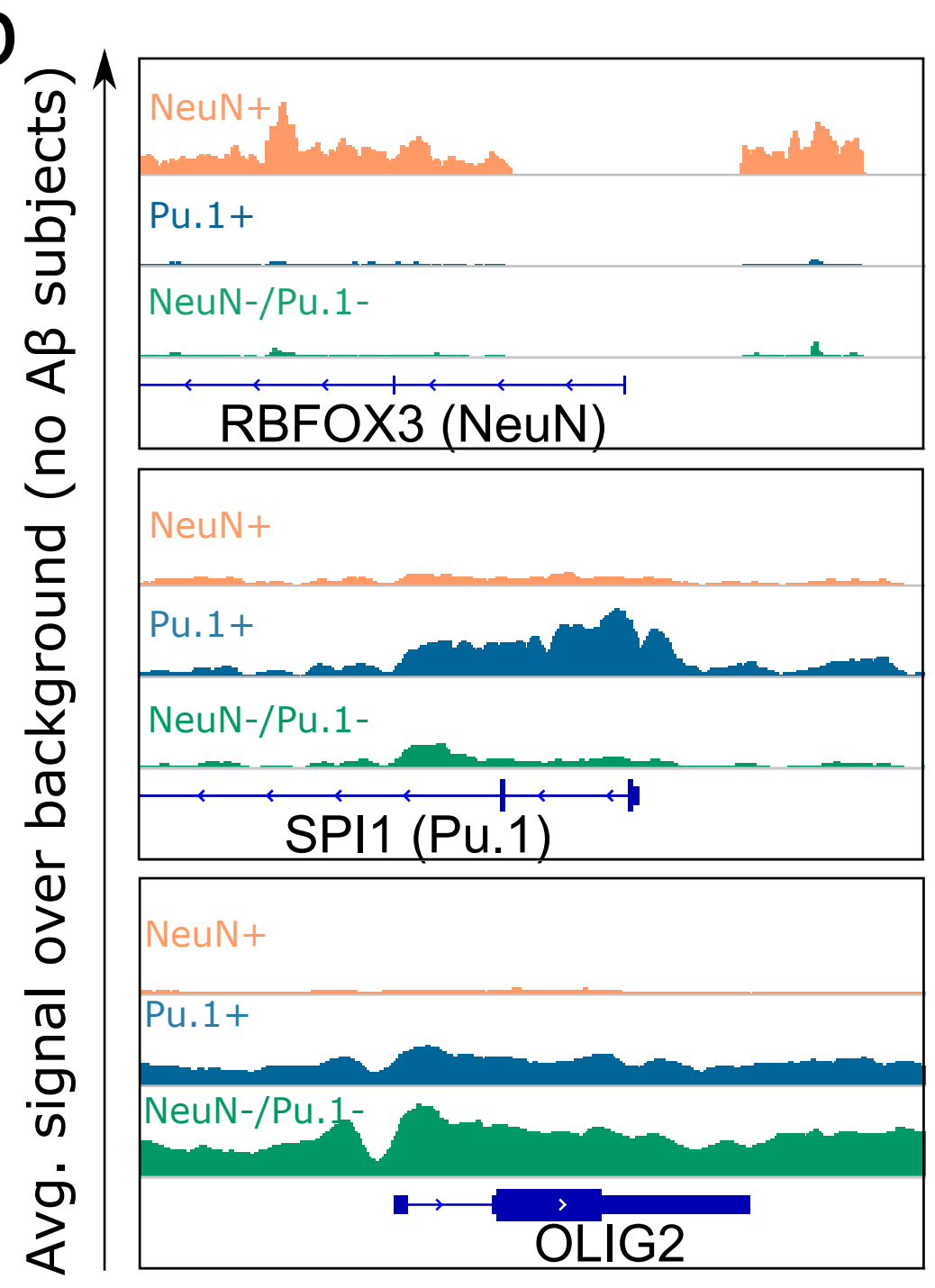

Single Cell Suspension and Fixation

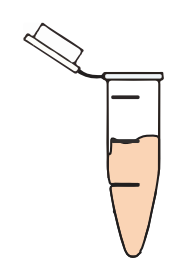

C

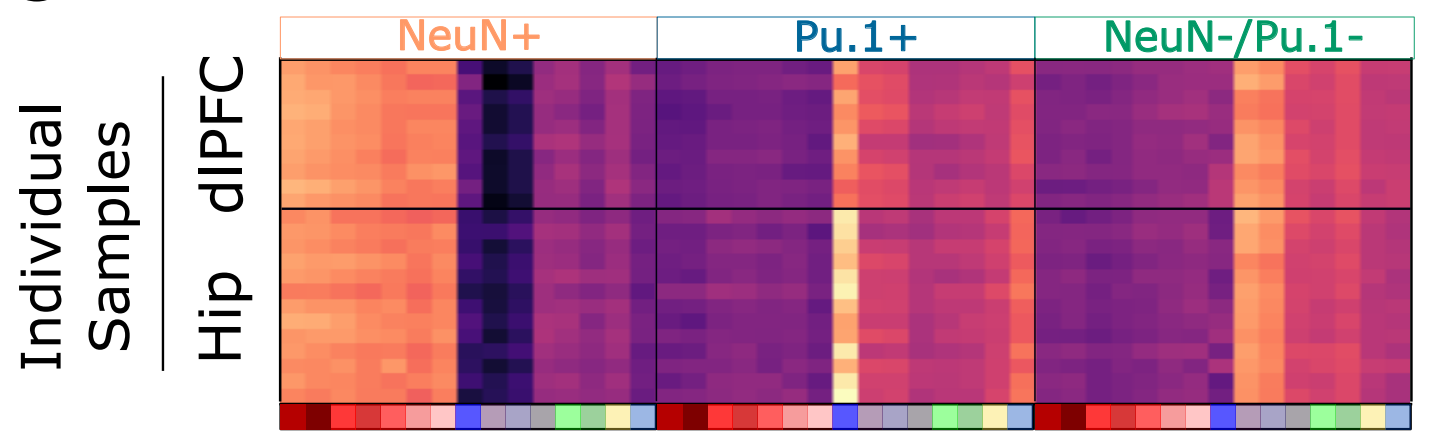

$$
\begin{array}{lll}
-2 & 0 & 2
\end{array}
$$

$\log 2 \mathrm{fc}$ (z-score)
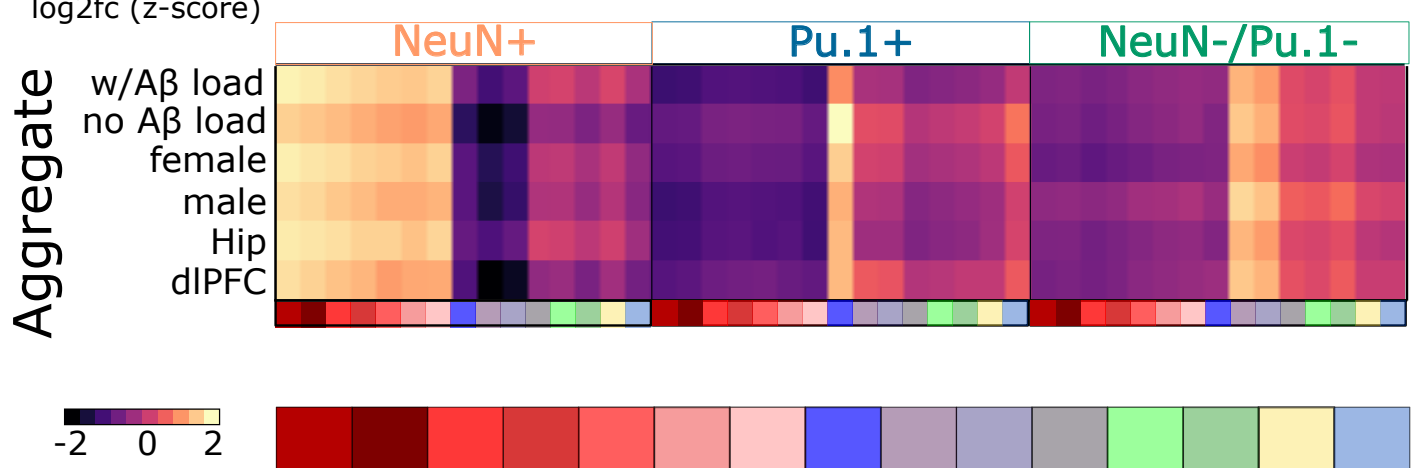

$\log 2 \mathrm{fc}$ (z-score)

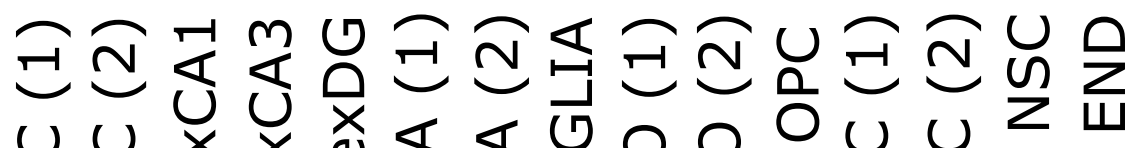

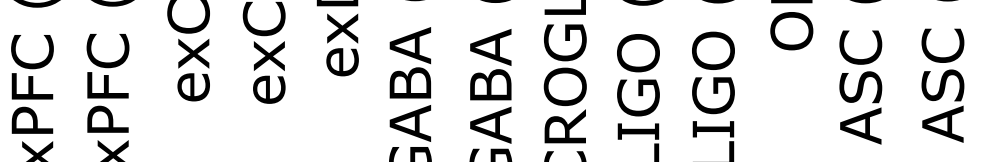

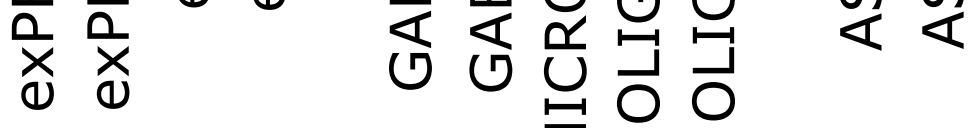

NeuN-

Neun+

PU.1+ PU.1 ע/

FANS

PU.1-
PU.1-

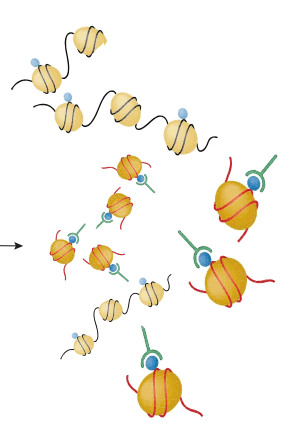

H3K27ac ChIP-seq

NeuN- 
$a$

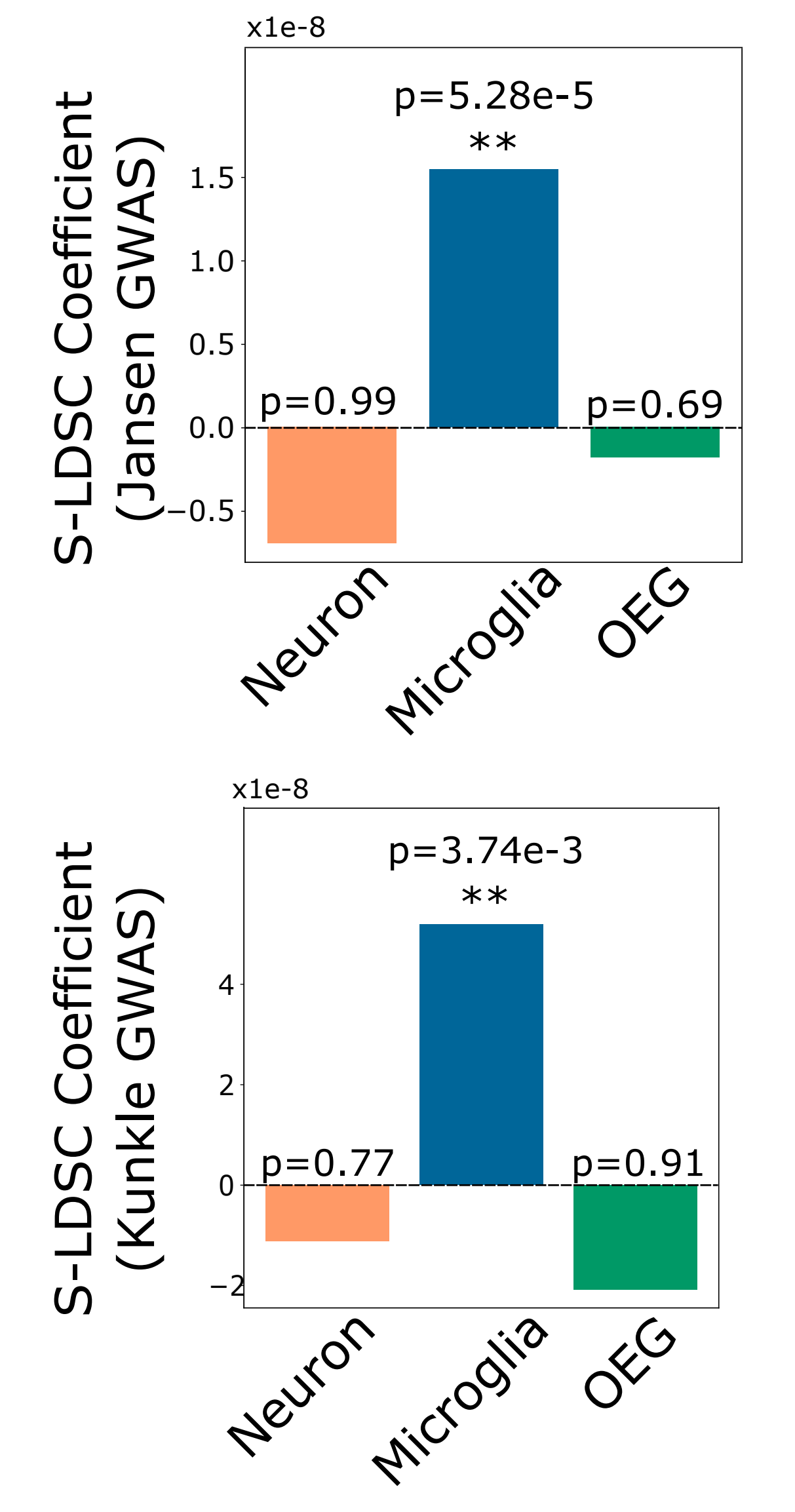

d

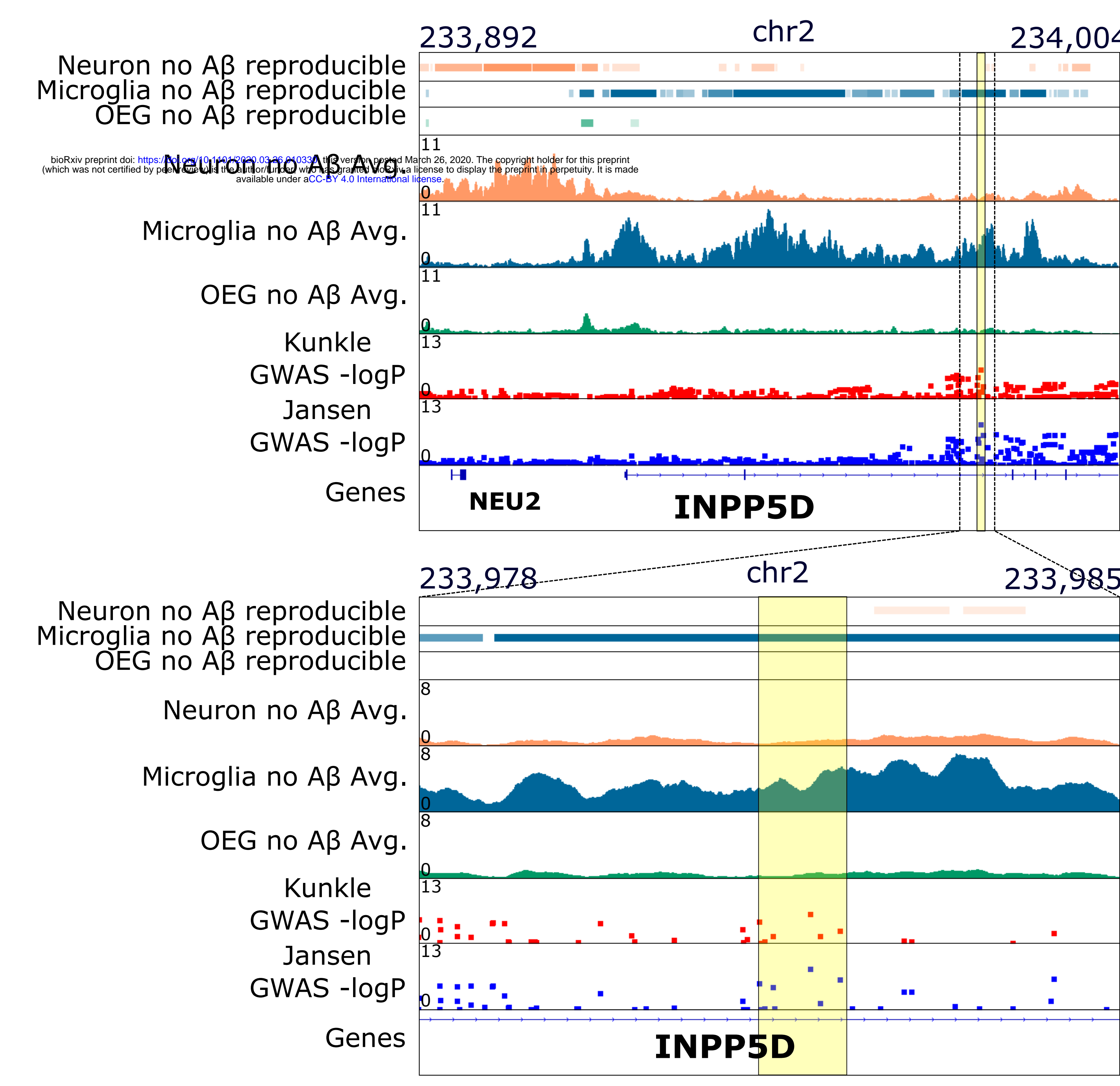

C $\quad$ Kunkle GWAS Sentinel SNPs

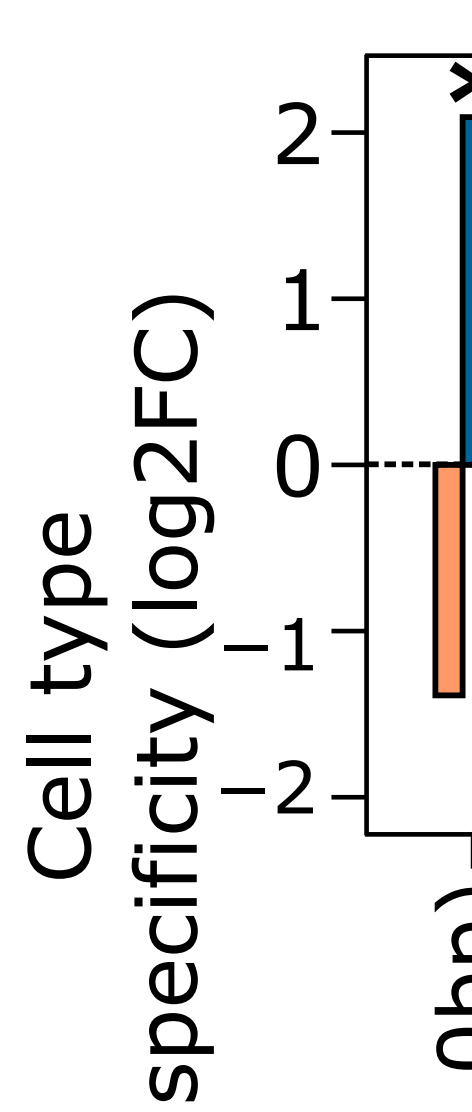

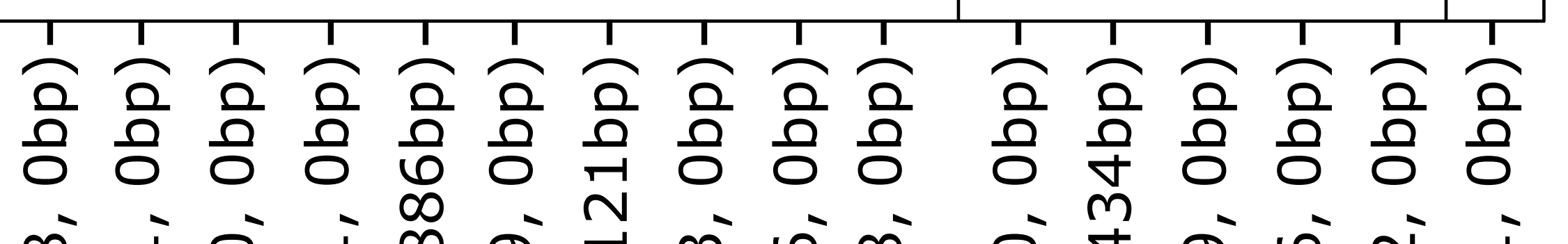

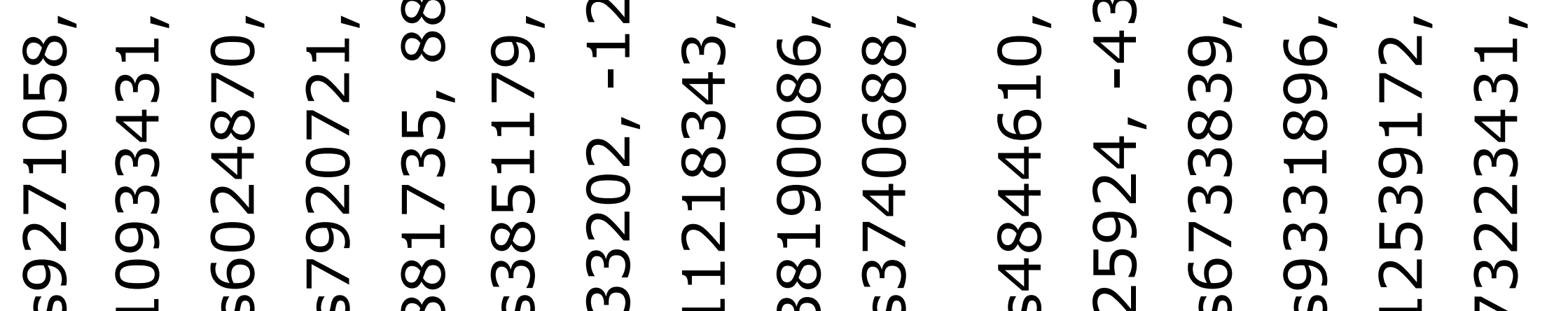

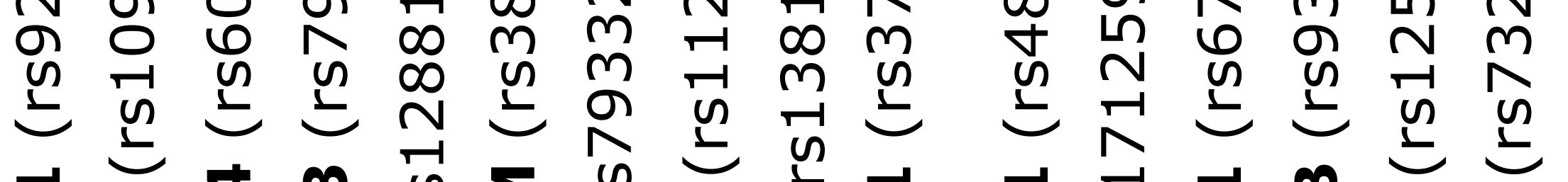

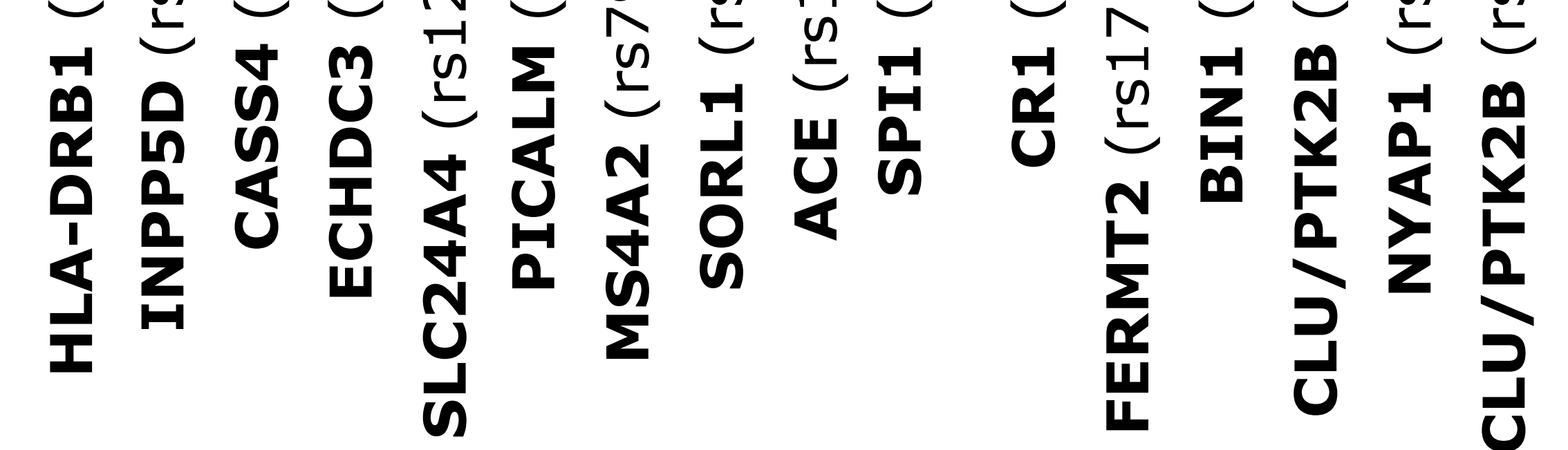

e
Jansen GWAS Sentinel SNPs

EOAD Genes

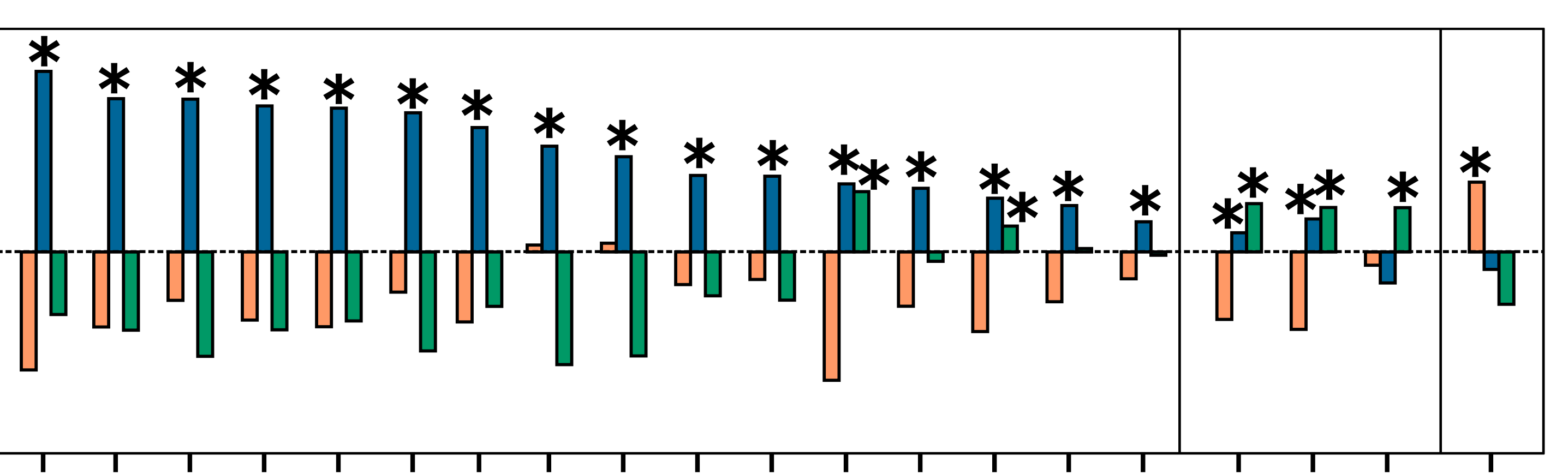

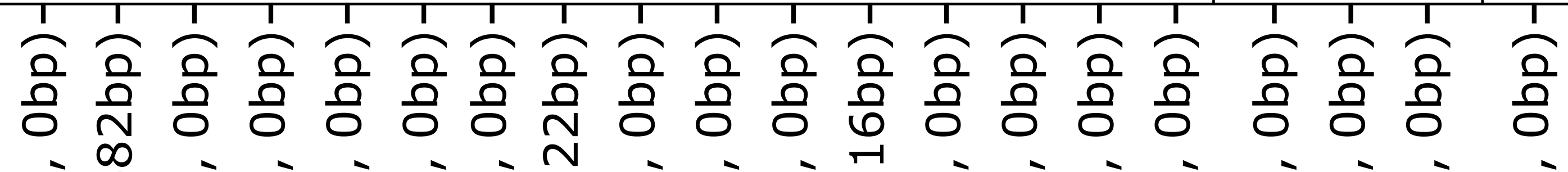

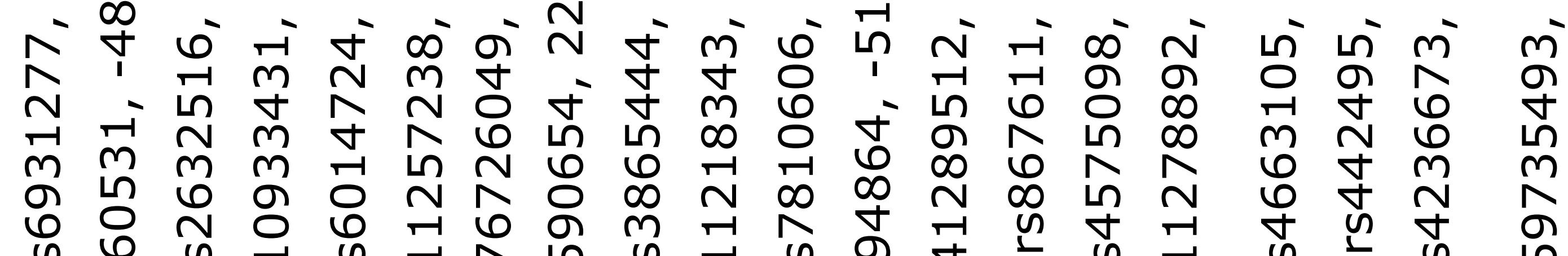

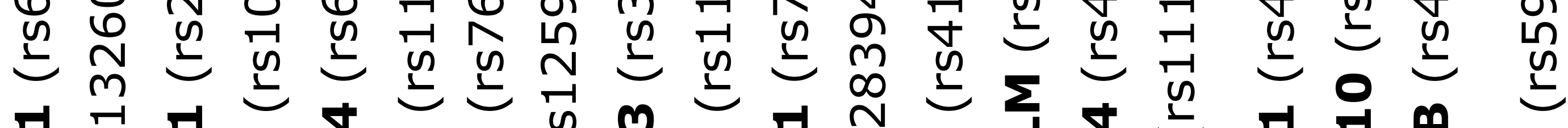

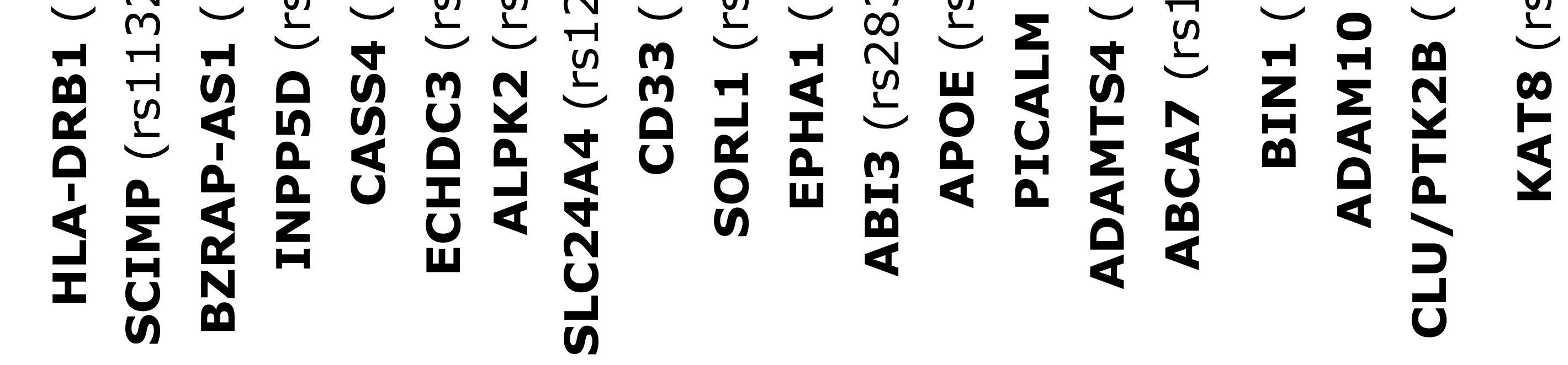

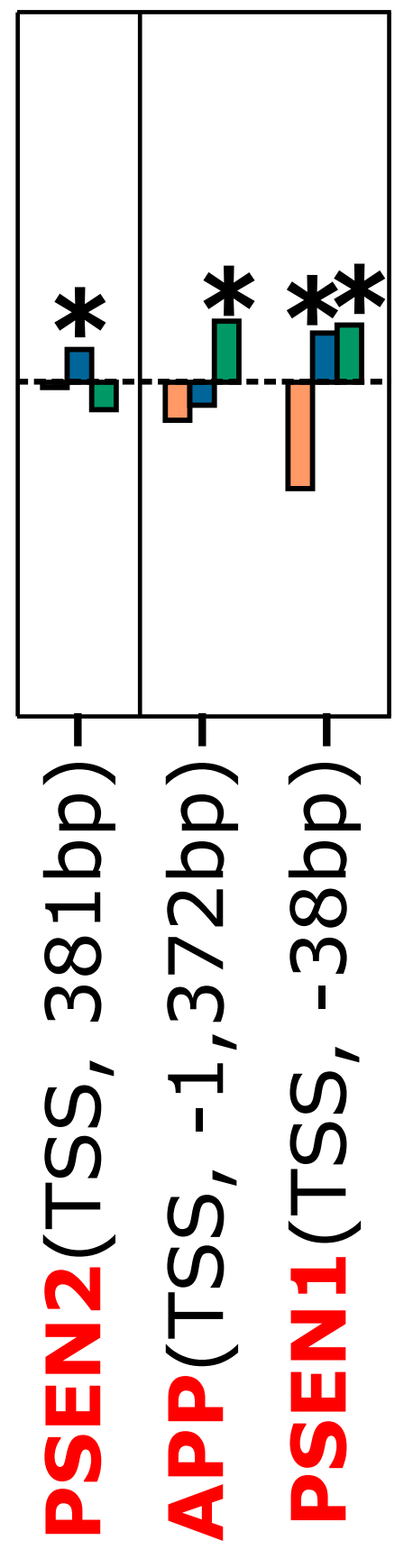

f

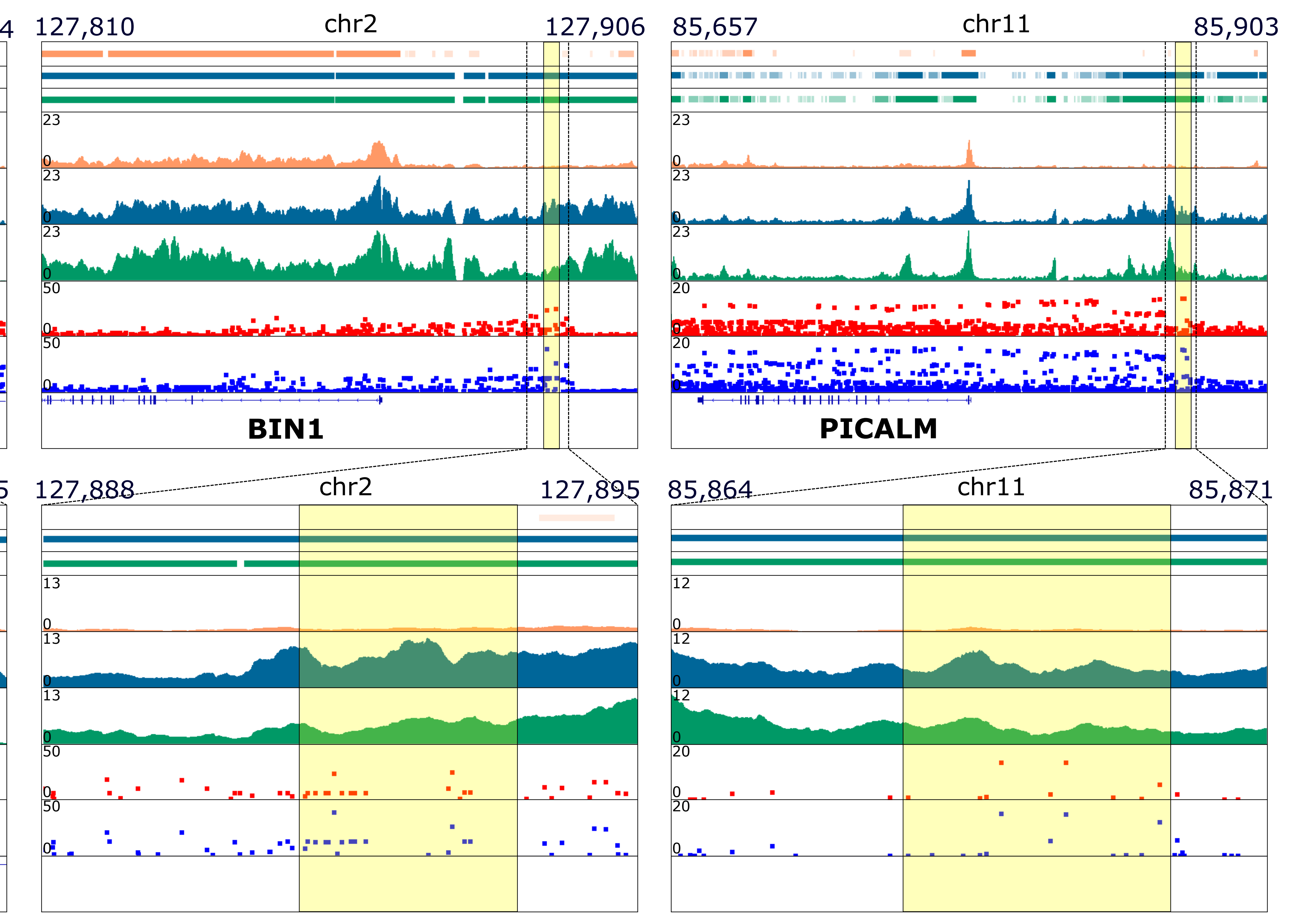



Num. Hyperacetylated

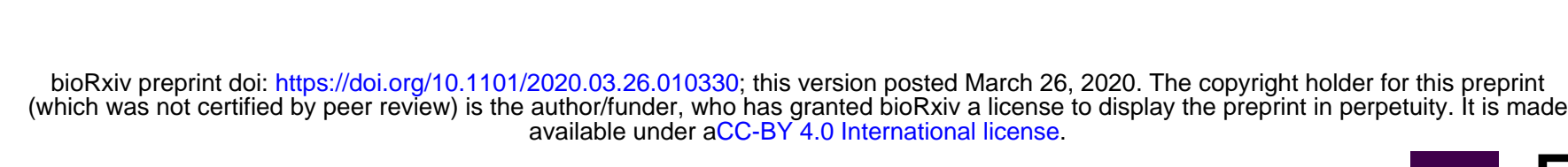

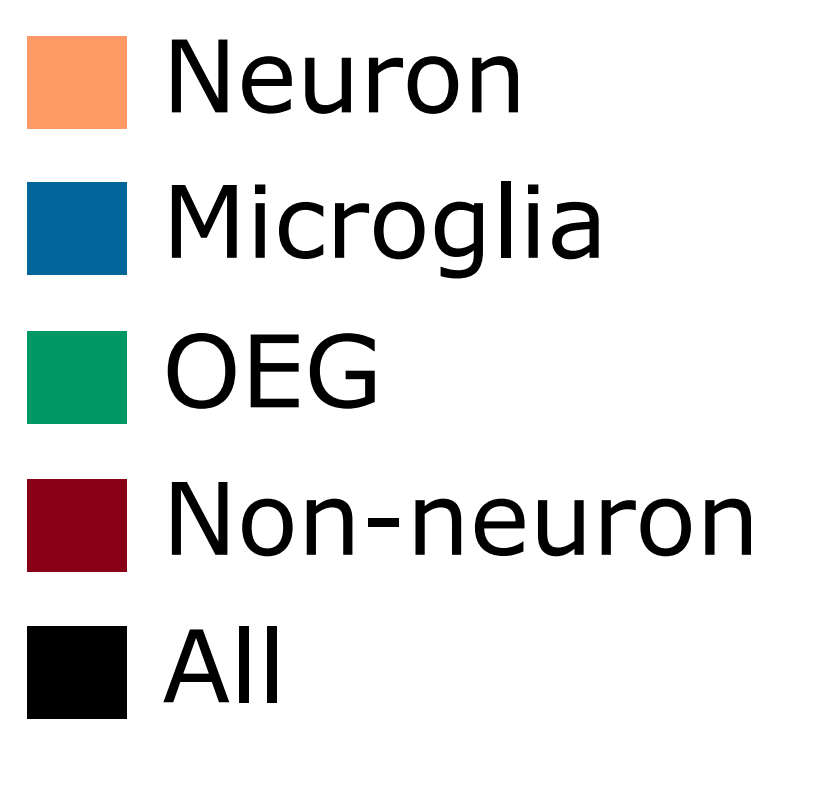

C

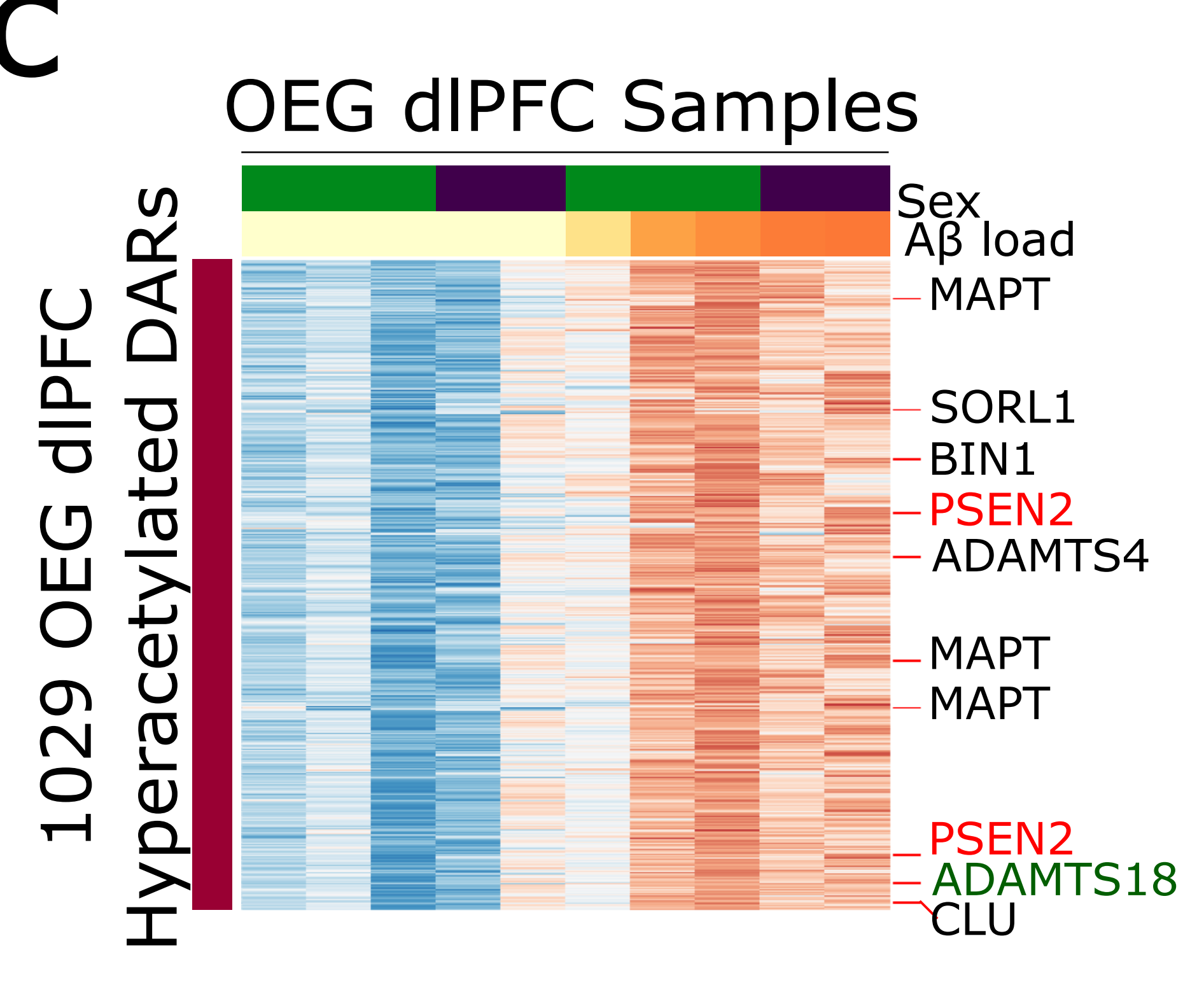

\section{dIPFC \\ Hippocampus \\ Both}

d

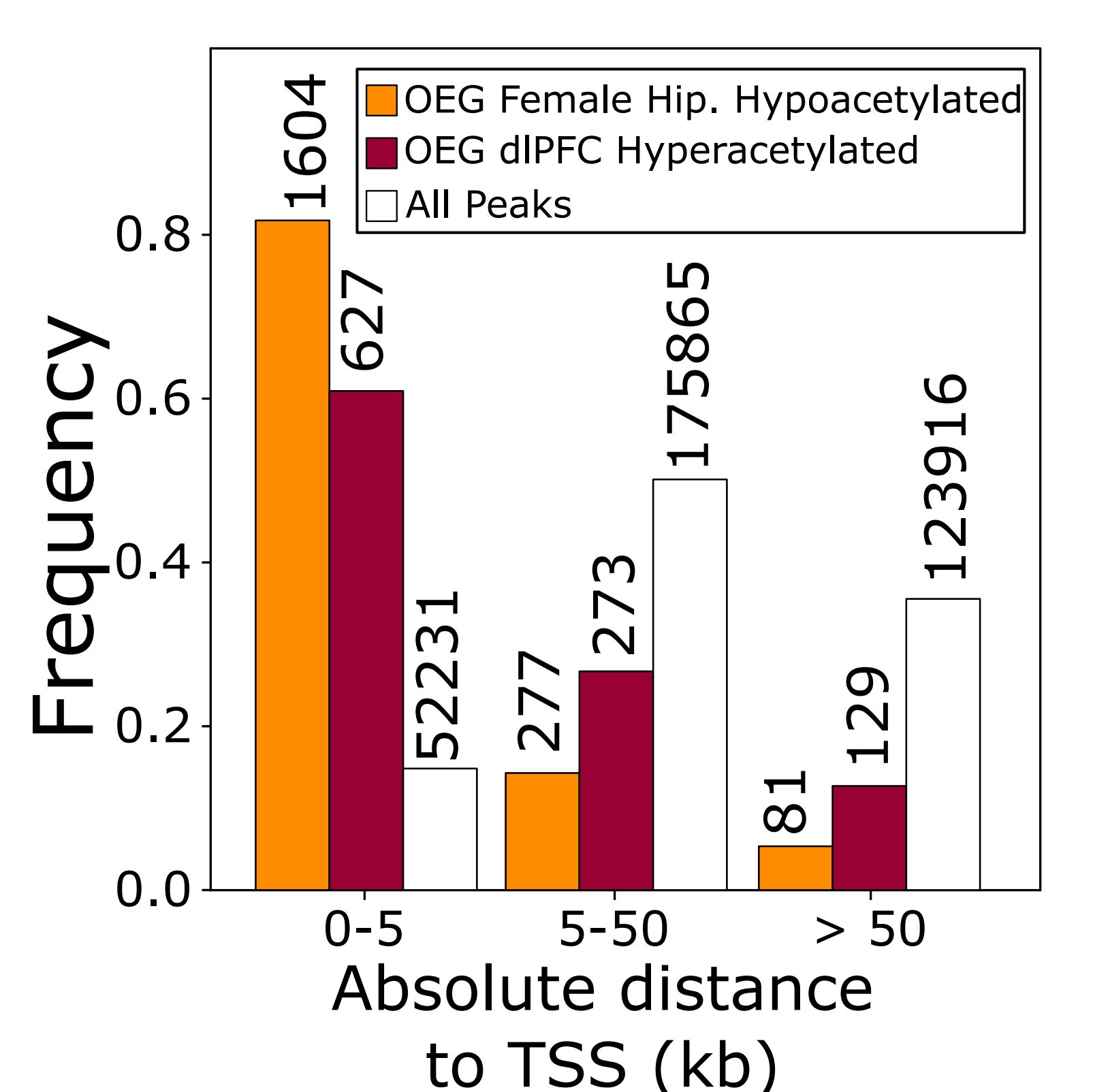

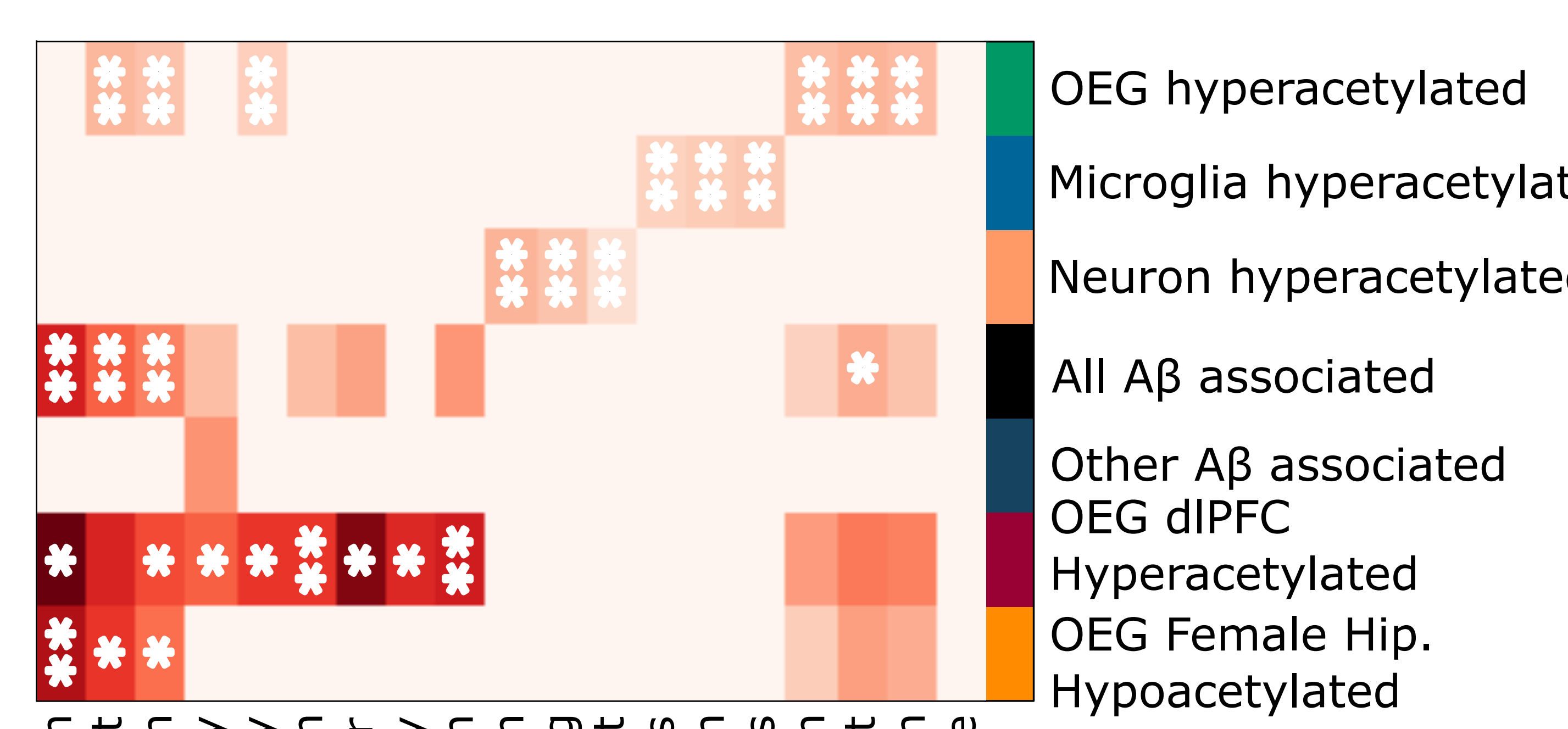

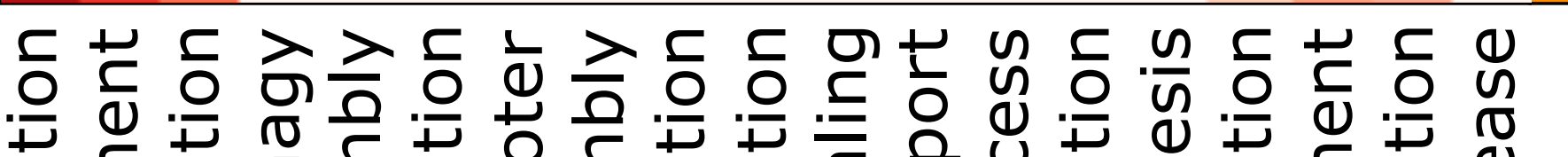

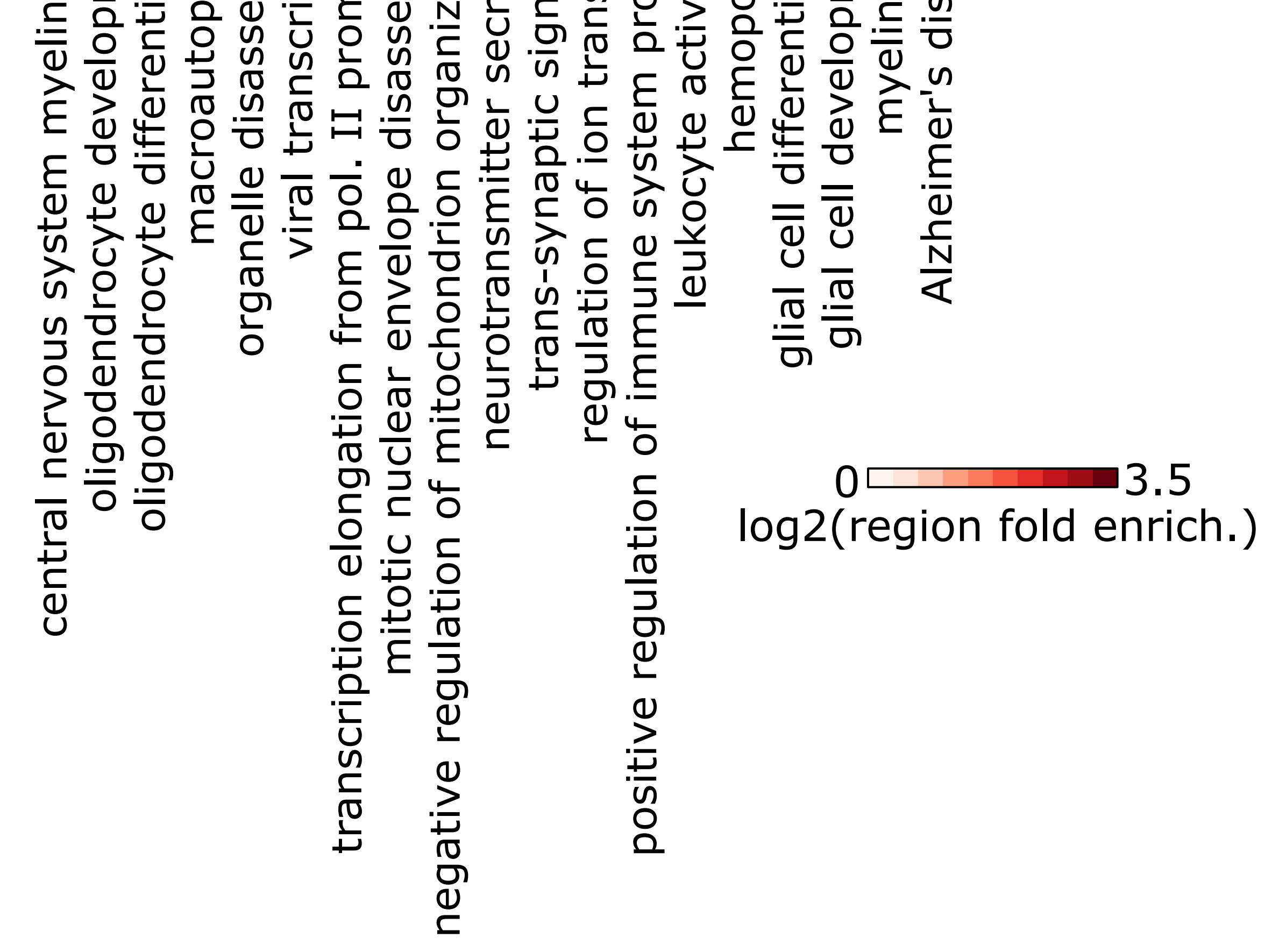

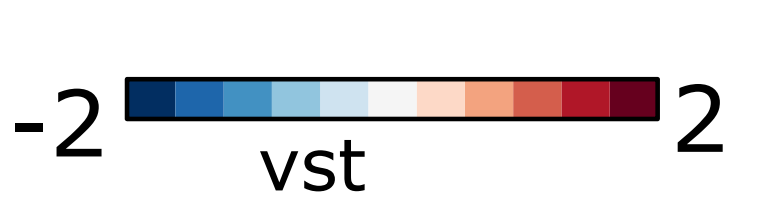

peak Z-score

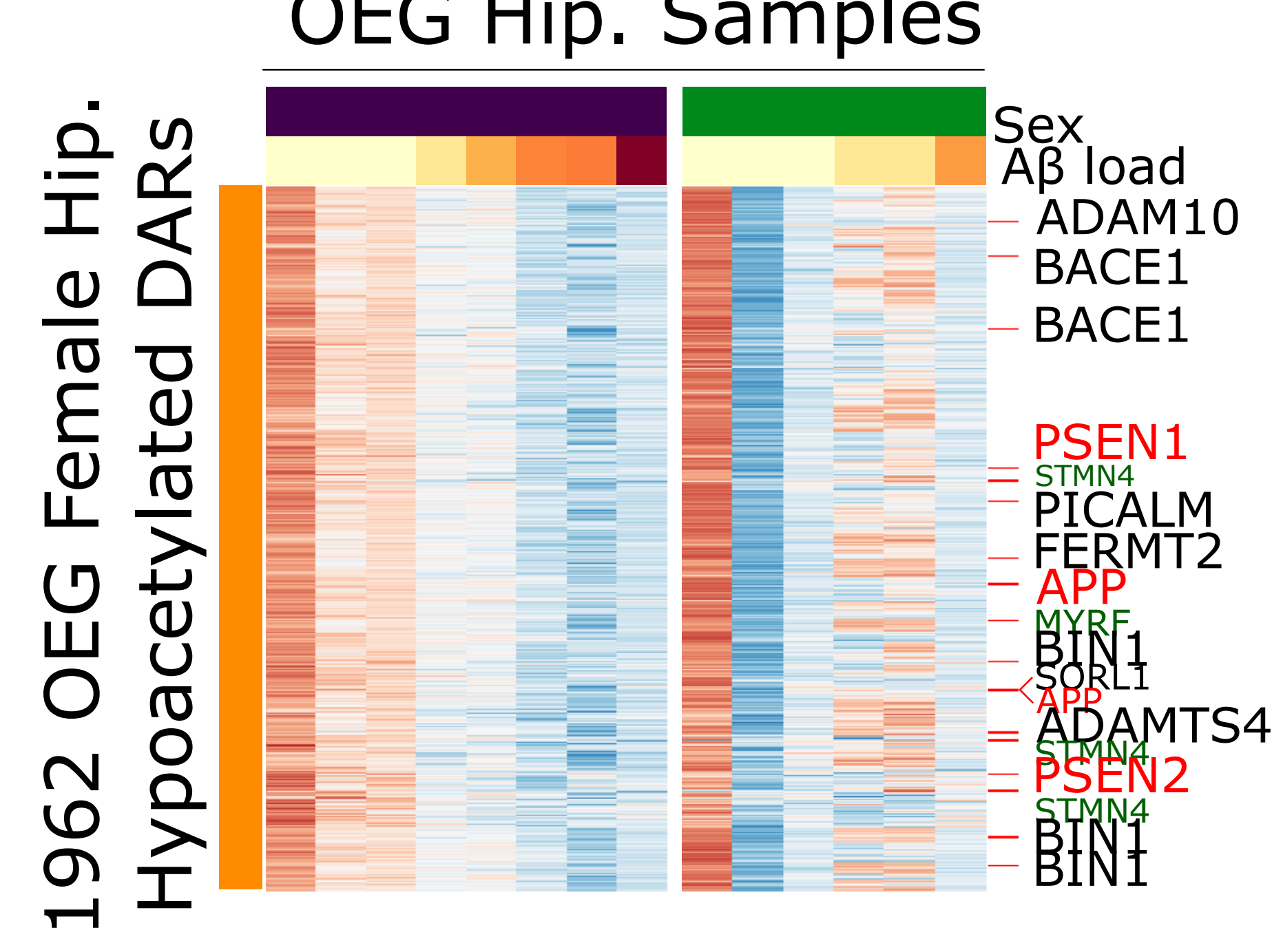

Male overall 14.8

\section{$a$} Sex
Cell Type
Brain Region

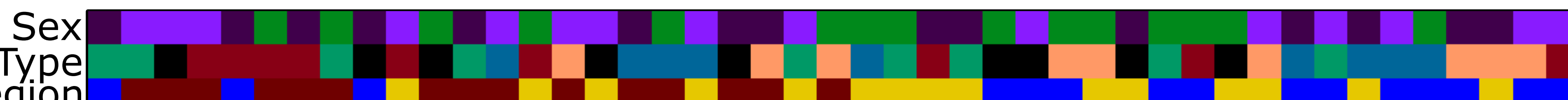
DAR set color code

$\begin{array}{ll}0 \underset{\text { Num. peaks }}{0} 1962 & \text { Female } \\ (\text { FDR q }<0.05) & \text { Male } \\ & \text { Both }\end{array}$

e b

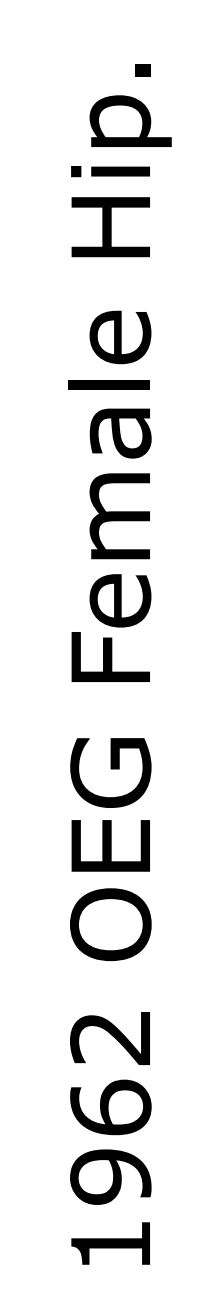




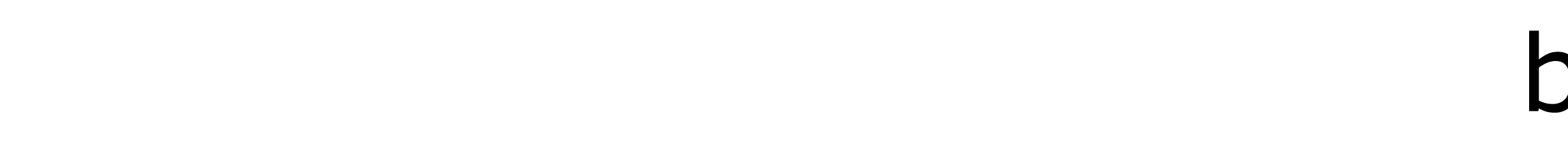

c $\quad d$ OEG Female Hip. Hypoacetylated OEG Female Hip. log2FC OEG dIPFC Avg. Signal ${ }^{36}$

OEG dIPFC Hyperacetylated OEG dIPFC log2FC All Peaks
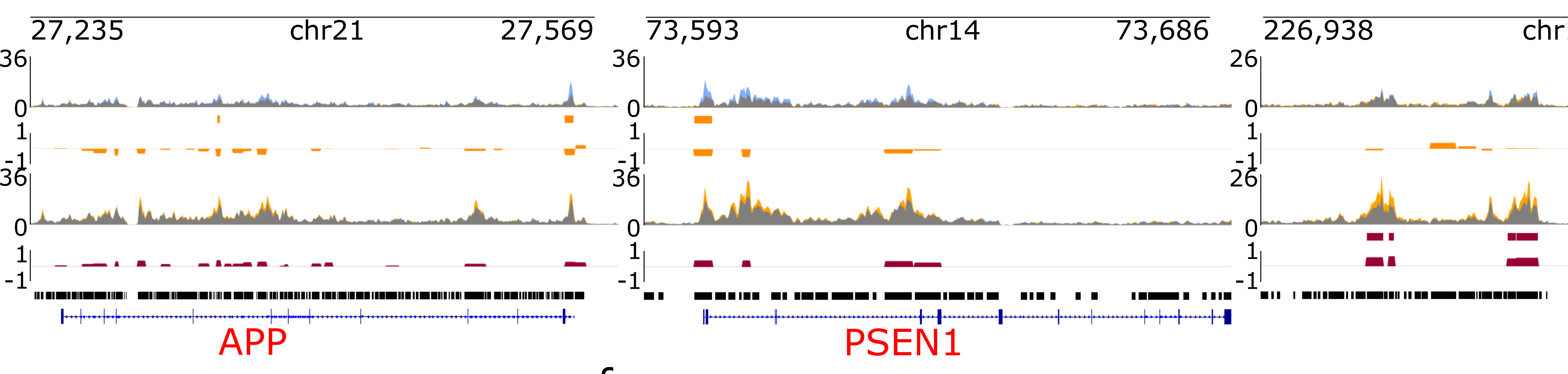

e

OEG Female Hip. Avg. Signal
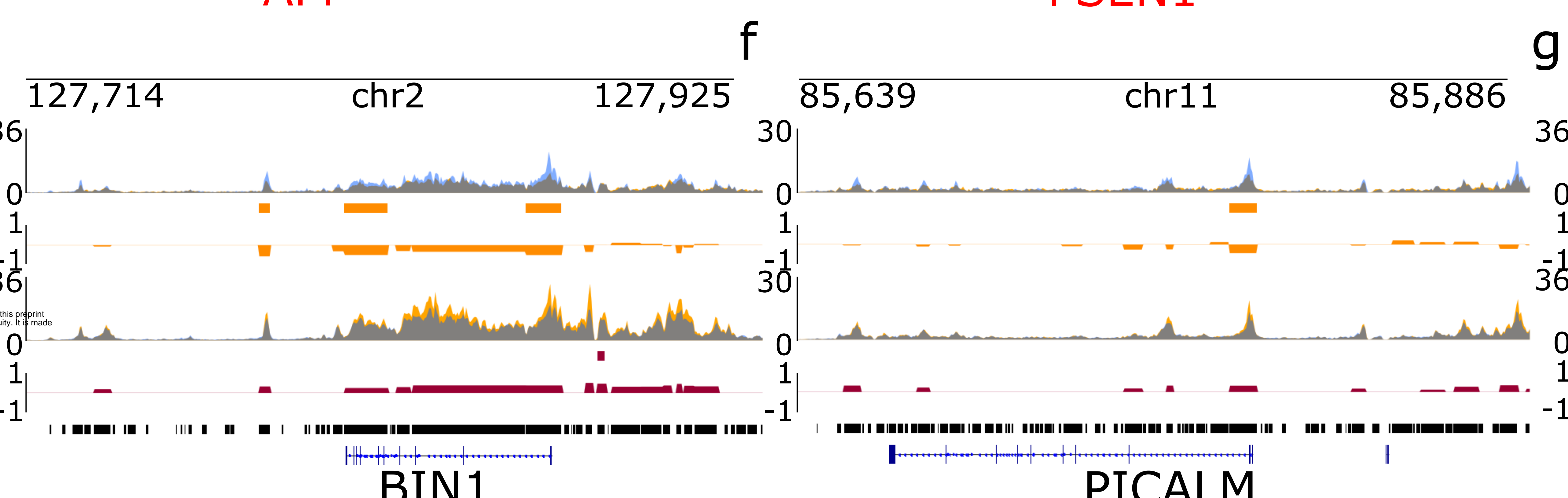

OEG Female

OEG

OEG dIPFC Avg. Signal 36

OEG dIPFC log2FC

$$
\text { All Peaks }
$$

\section{BIN1}

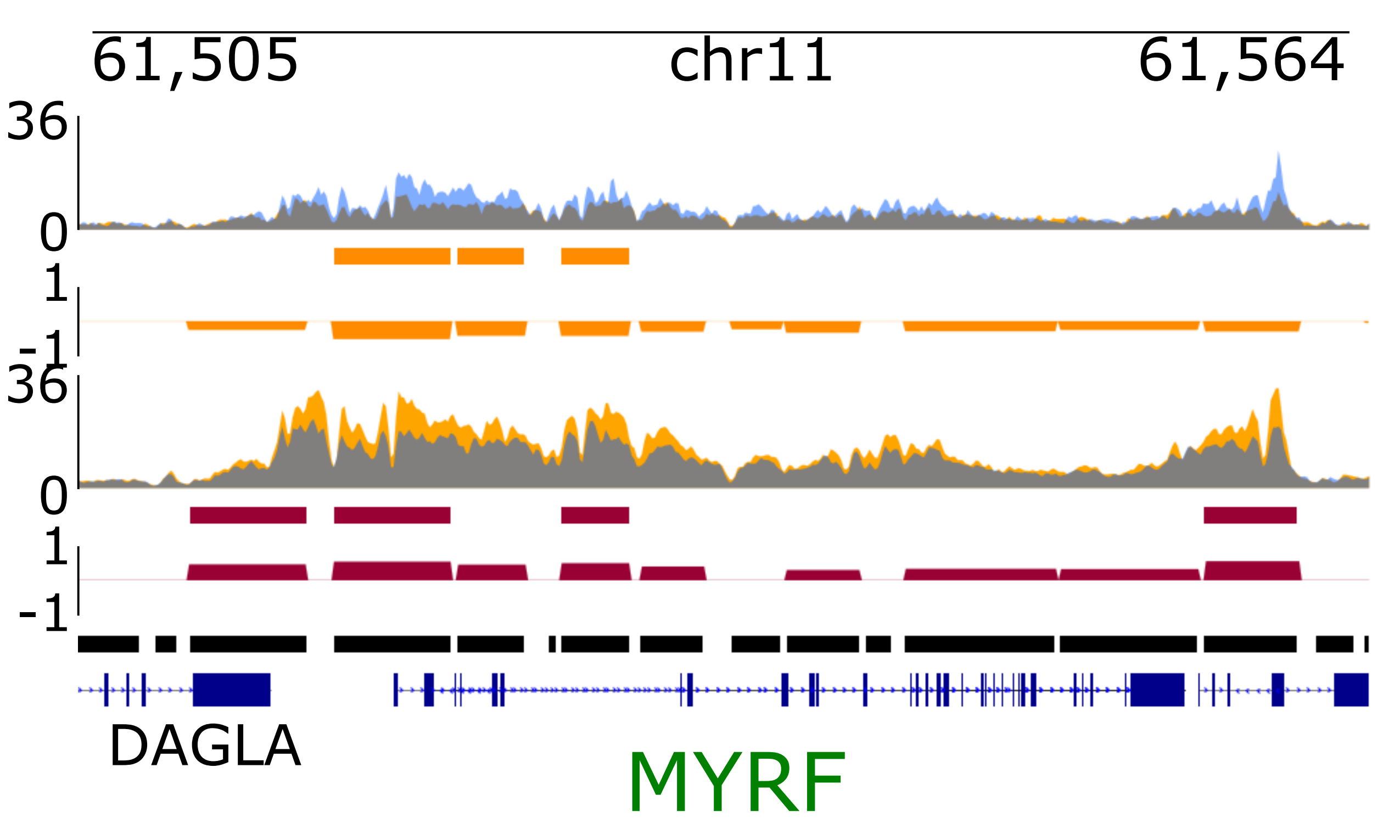

OEG Female Hip. Avg. Signal 36 OEG Female Hip. Hypoacetylated OEG Female Hip. log2FC OEG dIPFC Avg. Signal

OEG dIPFC Hyperacetylated OEG dIPFC log2FC All Peaks

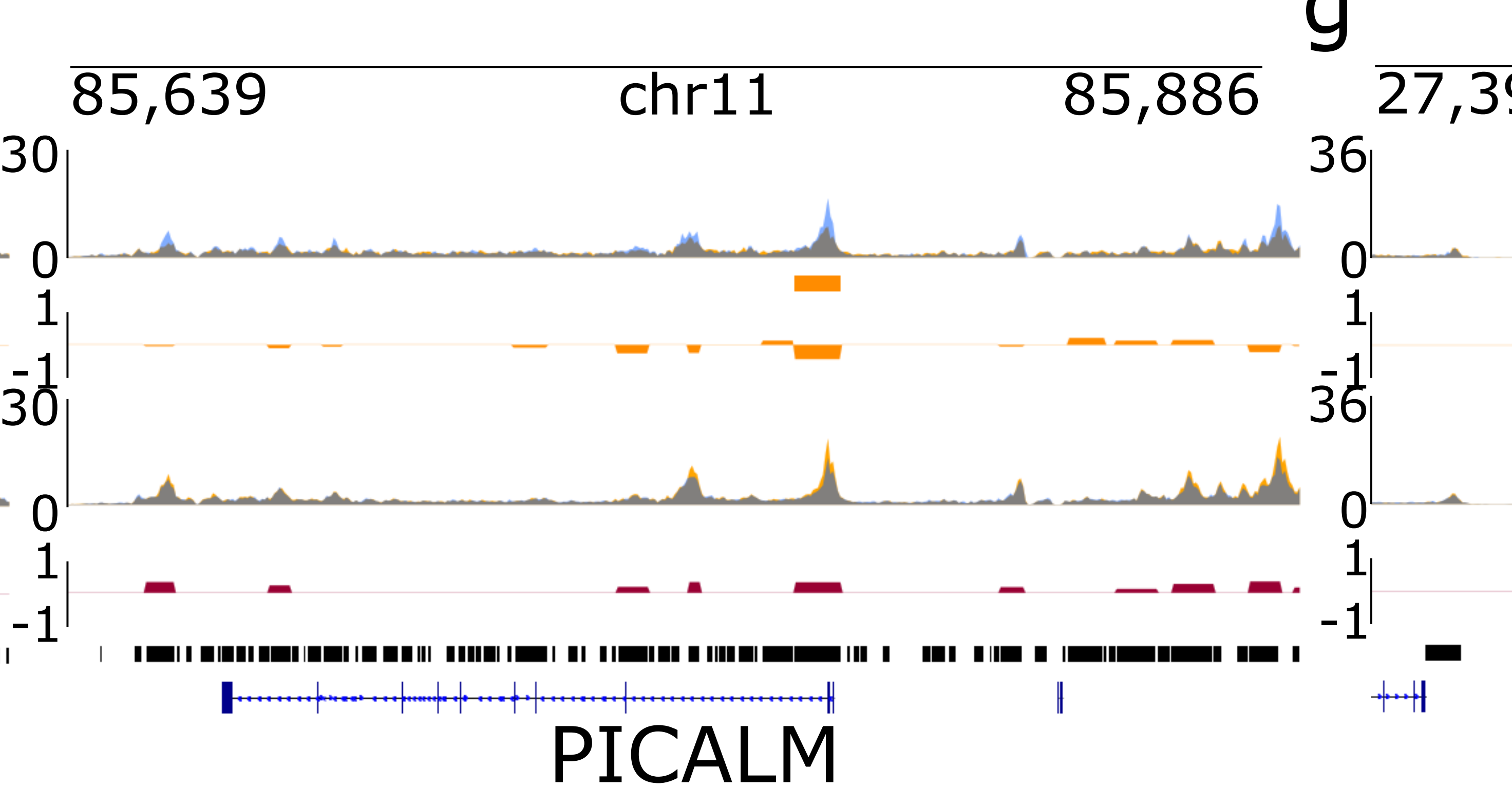

j

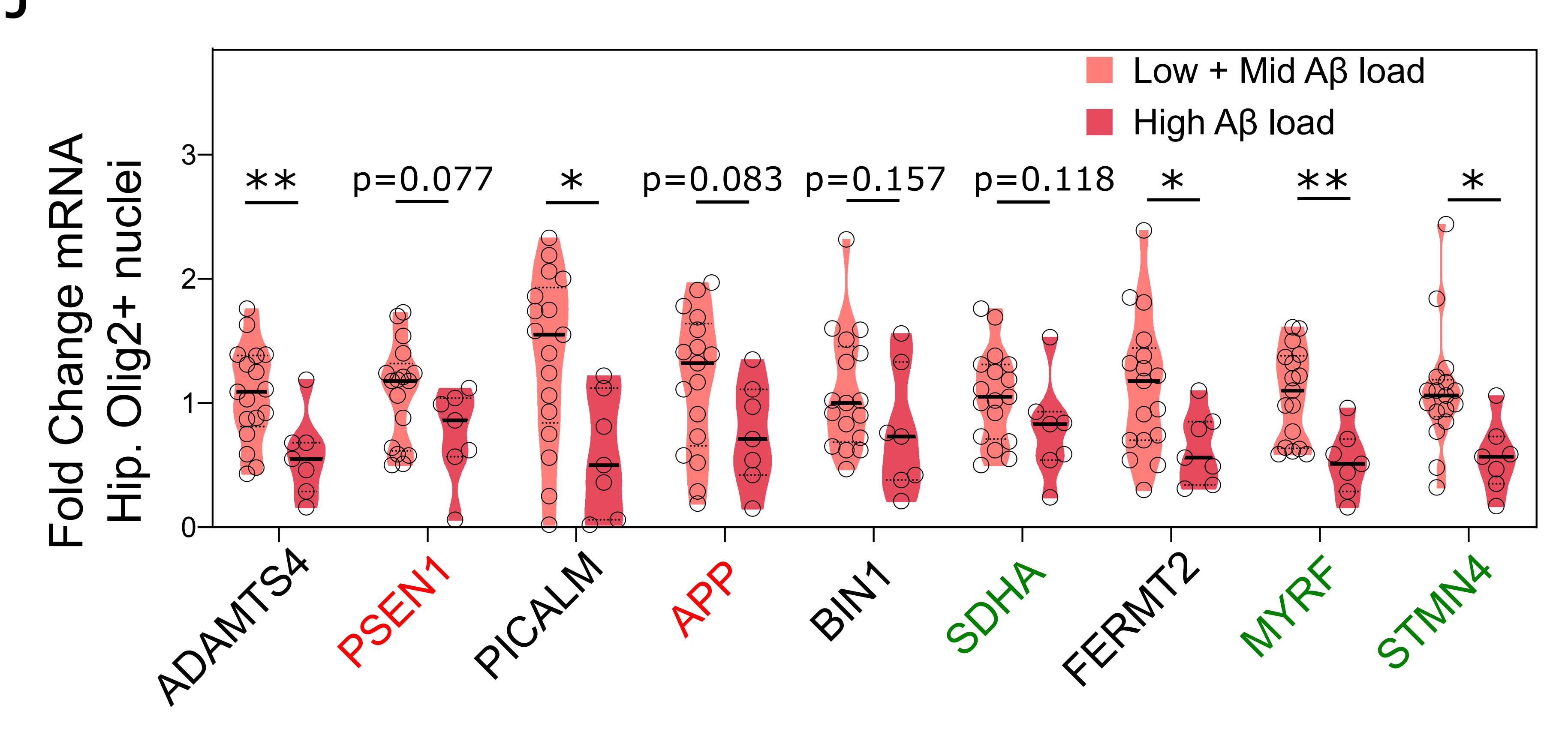

43,827<smiles>OCCOCC1CCCCC1</smiles>

PSEN2 $\mathrm{h}$

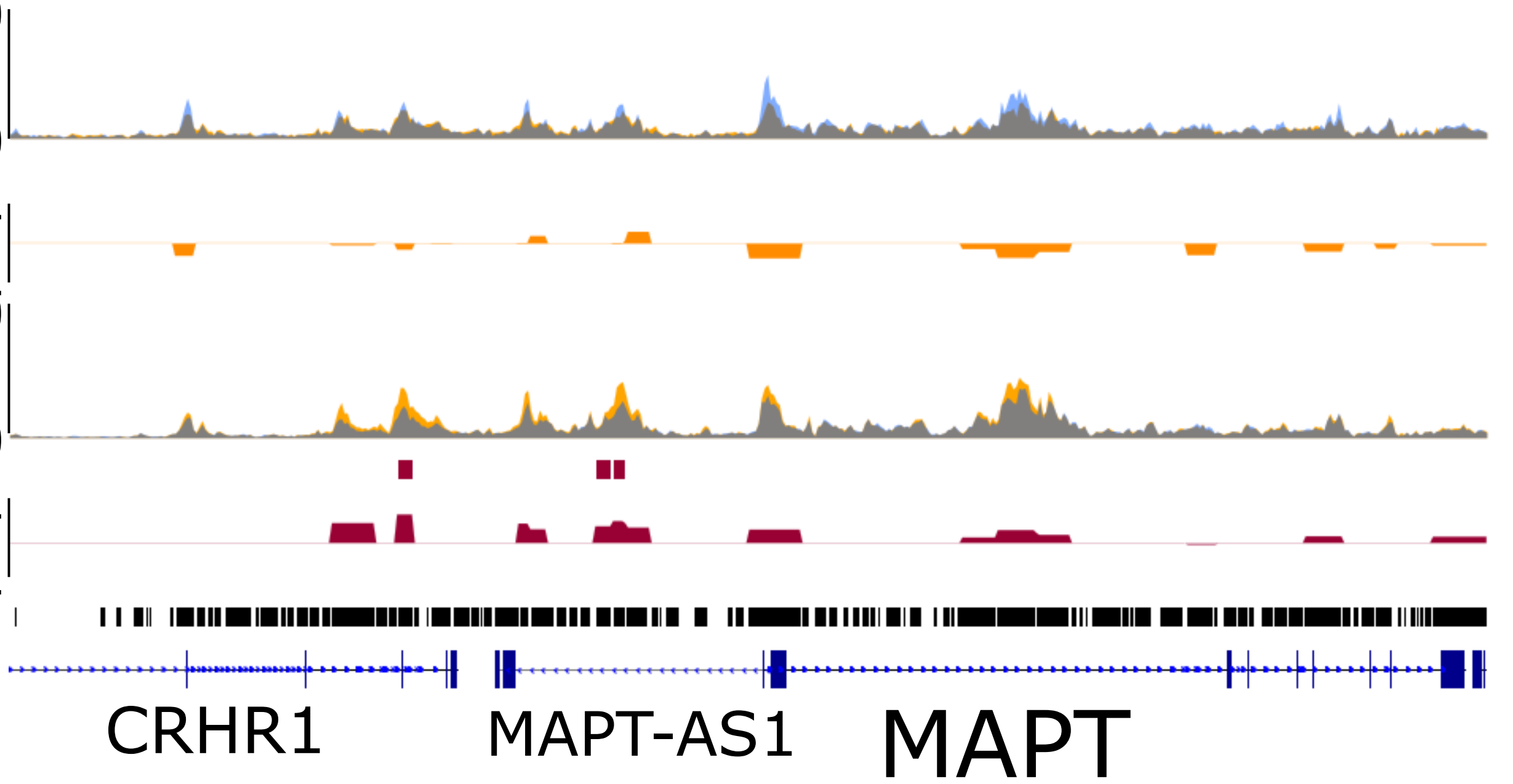

77,249

chr16

77,563
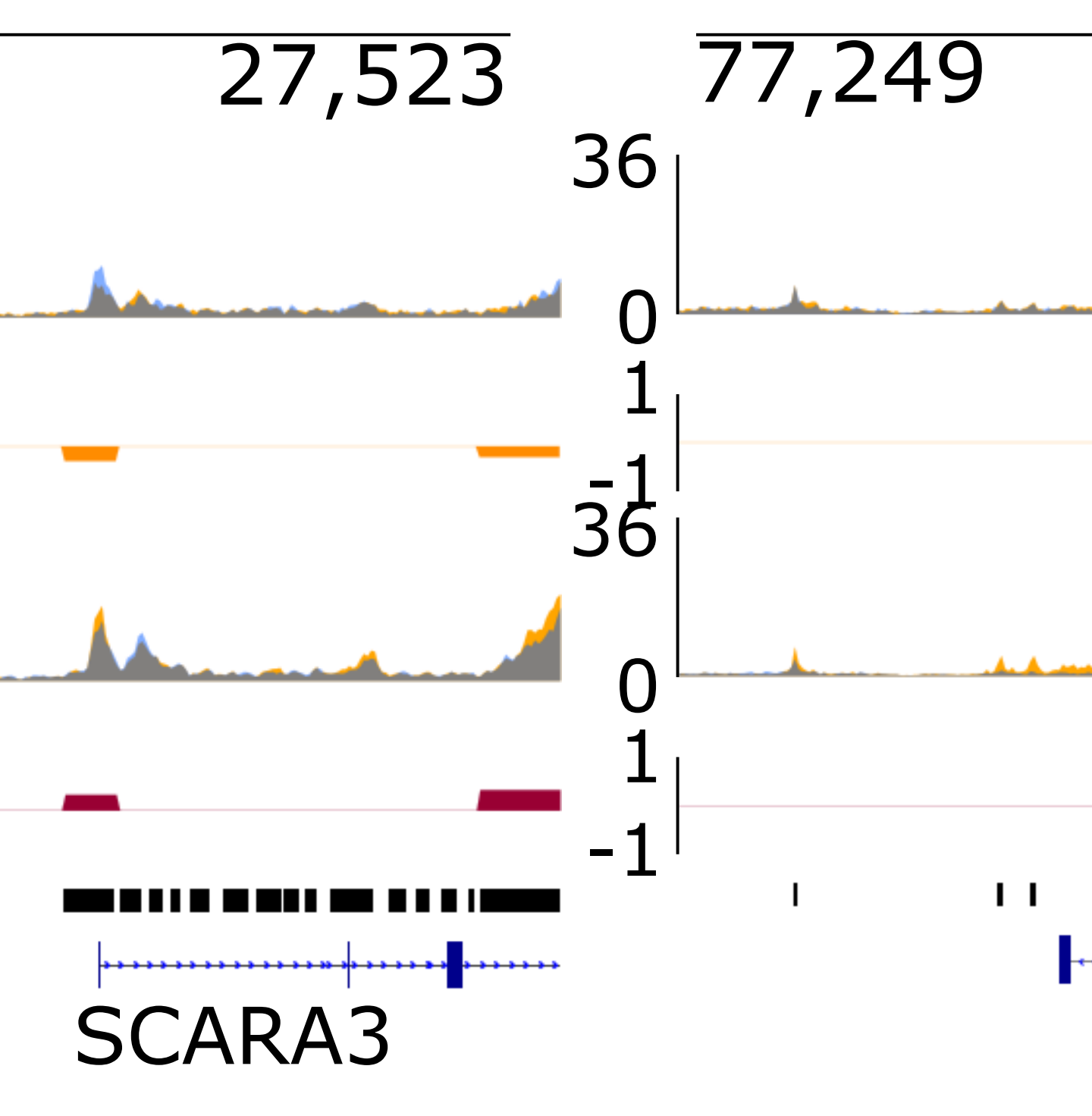

$\mathrm{k}$

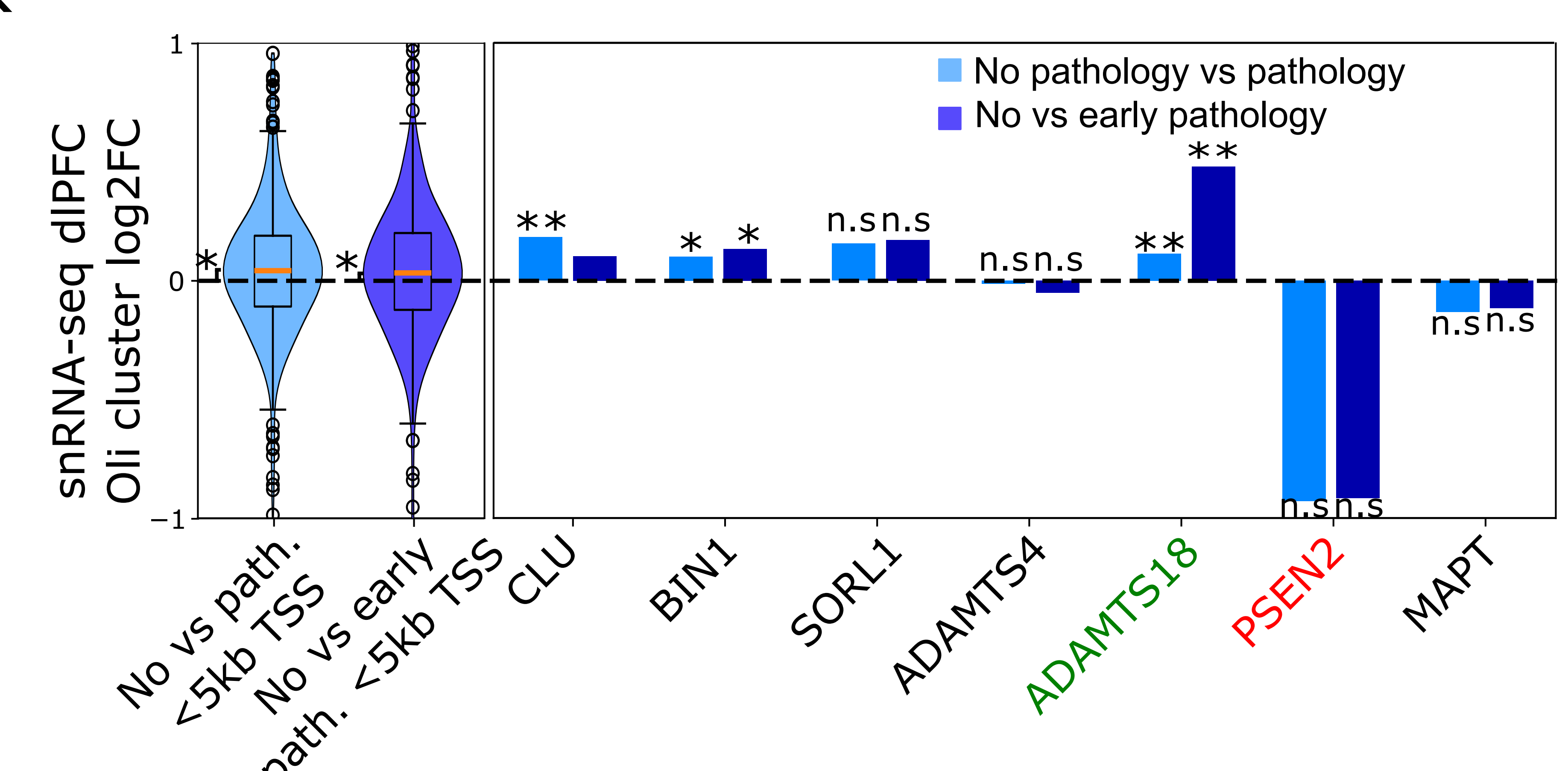

